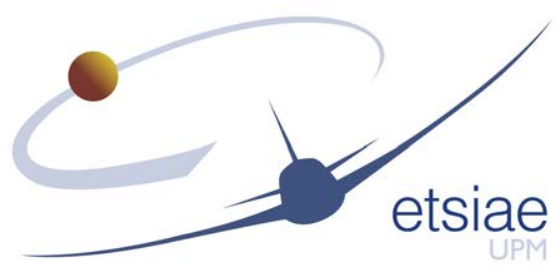

UNIVERSIDAD POLITÉCNICA DE MADRID

ESCUELA TÉCNICA SUPERIOR DE INGENIERÍA AERONÁUTICA Y DEL ESPACIO

\title{
WATER DROPLET DEFORMATION AND BREAKUP IN THE VICINITY OF THE LEADING EDGE OF AN INCOMING AIRFOIL
}

TESIS DOCTORAL

Adelaida García-Magariño García

Ingeniero Aeronáutico 
DEPARTAMENTO DE MECÁNICA DE FLUIDOS Y PROPULSIÓN AEROESPACIAL

ESCUELA TÉCNICA SUPERIOR DE INGENIERÍA AERONÁUTICA Y DEL ESPACIO

\section{WATER DROPLET DEFORMATION AND BREAKUP IN THE VICINITY OF THE LEADING EDGE OF AN INCOMING AIRFOIL}

TESIS DOCTORAL

Adelaida García-Magariño García

Ingeniero Aeronáutico

directed by

Angel Velazquez

Doctor Ingeniero Industrial

2016 


\section{POLITÉCNICA}

Tribunal nombrado por el Sr. Rector Magfco. de la Universidad Politécnica de Madrid, el día de. de $20 . . .$.

Presidente:

Vocal:

Vocal:

Vocal:

Secretario:

Suplente:

Suplente:

Realizado el acto de defensa y lectura de la Tesis el día ..de. de $20 \ldots$ en la E.T.S.I. /Facultad.

Calificación

EL PRESIDENTE

LOS VOCALES 


\section{Table of Contents}

ABSTRACT TII

RESUMEN EN ESPAÑOL . . . . . . . . . . . . . . . . . . III

RESUMEN EN INGLES . . . . . . . . . . . . . . . . . . . IV

1. INTRODUCTION 1

1.1. MOTIVATION ................... 1

1.1.1. Basic Aspects . . . . . . . . . . . . . . . . . . . 1

1.1.2. Technological Aspects . . . . . . . . . . . . . . 2

1.2. LITERATURE REVIEW . . . . . . . . . . . . . . . . 3

1.2.1. Literature Review on Droplet Deformation and Breakup . . . 3

1.2.2. Literature Review on Technological Application Aspects . . . 15

1.3. OBJECTIVES OF THE THESIS . . . . . . . . . . . . 22

2. DESCRIPTION OF THE EXPERIMENTAL SETUP 23

2.1. EXPERIMENTAL SETUP OF THE DROPLET DEFORMATION AND

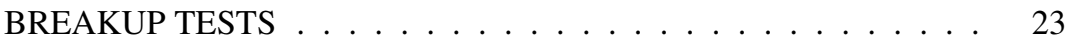

2.1.1. Rotating Arm Facility . . . . . . . . . . . . . . . . . 24

2.1.2. Airfoils Models . . . . . . . . . . . . . . . . . . . 25

2.1.3. Droplet Generator . . . . . . . . . . . . . . . . . . 25

2.1.4. Data Acquisition System . . . . . . . . . . . . . . . . 29

2.2. EXPERIMENTAL SETUP OF THE BASE FLOW MEASUREMENTS 32

2.2.1. PIV System . . . . . . . . . . . . . . . . . . . . 32

2.2.2. PIV Experimental Setup . . . . . . . . . . . . . . . . . 34

2.2.3. Data Dispersion . . . . . . . . . . . . . . . . . . 42

2.3. DATA PROCESSING SOFTWARE . . . . . . . . . . . . . 42

2.3.1. Pre-processing . . . . . . . . . . . . . . . . 42

2.3.2. Processing . . . . . . . . . . . . . . . 43

2.3.3. Uncertainty in the measurements . . . . . . . . . . . . 45

2.3.4. Computational Time . . . . . . . . . . . . . . . 47

2.3.5. Visualizing the data: Data Analysis _. . . . . . . . . 47

3. EXPERIMENTAL TESTING 49

3.1. BASE FLOW CHARACTERIZATION . . . . . . . . . . . . . 49

3.1.1. PIV Measurements . . . . . . . . . . . . . . . . . . . . 49

3.1.2. Characterization of the base flow . . . . . . . . . . 50

3.2. DROPLET DEFORMATION AND BREAKUP RESULTS . . . . . . 52

3.2.1. Experimental Set $1 \ldots \ldots \ldots \ldots$

3.2.2. Experimental Set $2 \ldots \ldots \ldots \ldots$ 
3.2.3. Experimental Set $3 \ldots \ldots \ldots$. . . . . . . . . . . 59

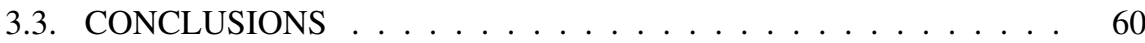

4. DATA REDUCTION USING HOSVD 63

4.1. INTRODUCTION TO DATA REDUCTION . . . . . . . . . . . . 63

4.2. HOSVD METHOD DESCRIPTION . . . . . . . . . . . . . 65

4.3. HOSVD ANALYSIS OF THE DATA . . . . . . . . . . . . 68

4.3.1. Experimental Data Set . . . . . . . . . . . . . . . . . 68

4.3.2. Application Case $1 \ldots \ldots$. . . . . . . . . . . . . . . 69

4.3.3. Application Case $2 \ldots \ldots \ldots$. . . . . . . . . . . 71

4.3.4. Application Case $3 \ldots \ldots \ldots$. . . . . . . . . . 71

4.3.5. Application Case $4 \ldots \ldots \ldots \ldots$. . . . . . . . . 71

4.3.6. Application Case $5 \ldots \ldots \ldots$. . . . . . . . . . 71

4.3.7. Discussion on the application cases 1 to $5 \ldots \ldots$. . . . . 71

4.3.8. Application Case 6 . . . . . . . . . . . . . . . . . 76

4.3.9. Application Case $7 \ldots \ldots$. . . . . . . . . . . . . 78

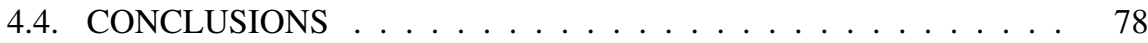

5. BREAKUP CRITERION 83

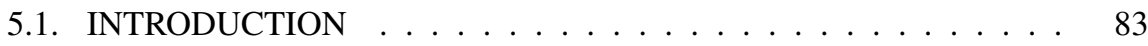

5.2. BREAKUP CRITERION PROPOSED: BAG AND STAMEN BREA-

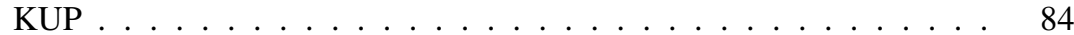

5.3. BAG BREAKUP . . . . . . . . . . . . . . . . . . . . 89

5.4. SHEAR BREAKUP . . . . . . . . . . . . . . . . . . . . . . . . 89

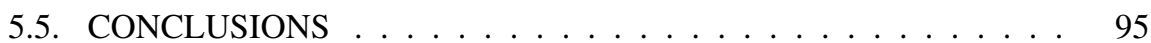

$\begin{array}{ll}\text { 6. CONCLUSIONS } & 97\end{array}$

$\begin{array}{ll}\text { BIBLIOGRAPHY } & 98\end{array}$ 


\section{ABSTRACT}

\section{RESUMEN EN ESPAÑOL}

La presente tesis ha tenido por objeto abordar el problema de la deformación y rotura de gotas debido a la presencia del campo fluido acelerado generado en las inmediaciones de un borde de ataque de un perfil aerodinámico. En la literatura hay numerosos estudios de deformación y rotura de gotas en otras condiciones, como campo fluidos de velocidad constante, cuyos trabajos experimentales se han llevado a cabo principalmente en instalaciones tales como tubos de choque o túneles de viento. Sin embargo la deformación y rotura de gotas en campos fluidos no estacionarios es un problema que no se había abordado todavía. Los campos fluidos no estacionarios, acelerados o decelerados, solo se habían estudiado para esferas solidas o para gotas lo suficiente pequeñas como para despreciar su deformación, siendo además este campo de investigación un campo todavía activo, ya que existen resultados experimentales contradictorios. Por tanto la principal novedad de este trabajo ha sido estudiar la deformación y rotura de gotas en un campo fluido no estacionario.

El problema de deformación y rotura de gotas en las inmediaciones de un perfil es de aplicación en el campo aeronáutico, en concreto, en el problema de formación de hielo en alas de aviones debido a la presencia de gotas sobre-enfriadas grandes (también conocidas como SLD). Cuando una gota sobreenfriada impacta en una superficie solida, es posible que se forme hielo en dicha superficie siendo la cantidad y la forma de hielo formado dependiente entre otros factores del tamaño de la gota y del lugar de impacto. Si las gotas se rompiesen antes de llegar a impactar en el ala, como de hecho se ha observado que ocurre, el tamaño de las gotas a impactar cambia bruscamente, lo que podría modificar la forma de hielo en el ala respecto al caso en que las gotas no se rompen. Asimismo, y aunque las gotas no se rompan, el hecho de que se deformen significa que modifican su resistencia lo que se traduce en una variación de su trayectoria y consecuentemente del lugar de impacto. El hecho de que se forme hielo en las superficies aerodinámicas del avión es extremadamente peligroso, ya que modifica la sustentación y la resistencia de las mismas, pudiendo ocasionar accidentes.

El problema se abordó de forma experimental realizándose varias campañas de ensayos en las instalaciones del brazo rotatorio de INTA estudiándose gotas de diámetros entre 300 ţm y $3.6 \mathrm{~mm}$. En dichos ensayos, se montó un modelo en el extremo del brazo rotatorio de forma que se alcanzaron velocidades del modelo de hasta $90 \mathrm{~m} / \mathrm{s}$. Las gotas, en forma de chorro, se dejaron caer en la trayectoria del modelo que se acercaba a las mismas con velocidad constante. Se utilizaron tres modelos de distinto tamaño (radio del borde de ataque de $0.103 \mathrm{~mm}, 0.070 \mathrm{~mm}$ y $0.029 \mathrm{~mm}$ ) y cinco velocidades de modelo $(50 \mathrm{~m} / \mathrm{s}, 60 \mathrm{~m} / \mathrm{s}, 70 \mathrm{~m}=\mathrm{s}, 80 \mathrm{~m} / \mathrm{s}$ y $90 \mathrm{~m} / \mathrm{s})$. Por medio de la técnica de fotografía de sombras, se grabaron las imágenes de vídeo de la deformación y la rotura 
de las gotas. El campo fluido generado por los modelos se midió previamente usando la técnica PIV. Un software de análisis fue desarrollado para obtener datos cuantitativos de las imágenes sobre la deformación de las gotas y la rotura.

Una vez realizado los ensayos, en primer lugar, se realizó un estudio de caracterización del campo fluido generado por el modelo en las gotas, obteniéndose los principales parámetros de flujo implicados en el problema de la deformación y la ruptura de las gotas. Una ley de escala se obtuvo para la deformación. Después, se aplicó el método HOSVD de reducción de datos al problema con el objetivo de profundizar en una visión de la física subyacente. Se construyeron dos tensores: uno que contenía la información de la deformación y otro que contenía el tiempo de ruptura. Estos tensores se utilizaron para extrapolar fuera del tensor y para interpolar en el interior. Y, por último, se propone un criterio de rotura para este tipo de campos fluidos. Las principales conclusiones obtenidas se cuentan en los siguientes párrafos.

En la mayoría de los casos analizados durante las campañas experimentales, el tipo de ruptura fue tipo 'bag y stamen', excepto en unos pocos casos que se encontró tanto rotura tipo 'bag' como rotura tipo 'shear'. Los efectos no estacionarios debido a la velocidad y aceleración crecientes han demostrado jugar un papel fundamental en los procesos de deformación y rotura de las gotas. Se encontró además que si el cuadrado del tiempo de residencia de la gota por la aceleración se mantiene constante, la deformación de gotas (su relación de aspecto instantánea) depende únicamente de la velocidad de deslizamiento. Esto sugiere que el problema es, al menos, gobernado por un parámetro que implica dos tiempos característicos: el tiempo característico de la variación del campo fluido y el tiempo de residencia de las gotas.

Esta tesis pretendía únicamente ser el primer paso en un largo camino hasta poder generar métodos de ingeniería fiables para poder predecir el comportamiento de gotas en las proximidades de las alas de los aviones. Por tanto resultaba interesante obtener bases de datos fiables que pudieran estar disponibles y ser utilizadas en el desarrollo de algoritmos para este fin. Debido a su naturaleza propia, los experimentos involucrados son complejos y caros. En este contexto, el método de descomposición en valores singulares de alto orden (conocido como HOSVD) ha demostrado ser un método bastante adecuado de reducción de datos para este problema. La razón es que permite la generación de bases de datos limpias y densificadas (que se obtienen de conjunto limitado de experimentos) que se pueden usar fácilmente para fines de desarrollo del modelos numéricos.

Por último, se ha propuesto un nuevo criterio de rotura para predecir la rotura tipo 'bag and stamen', criterio que ha demostrado ser válido para todo el rango de casos experimentales analizados en esta tesis. Este criterio de rotura depende de un tiempo adimensional que es la relación del tiempo característico de deformación de las gotas y el tiempo característico de la variación del campo fluido.

\section{RESUMEN EN INGLES}

The problem of droplet breakup in the vicinity of the leading edge of an airfoil has been addressed. The aim of this thesis was to study the problem of the deformation and breakup of a droplet that is immersed in a flow field where the velocity and the acceleration that the droplet senses increases continuously. This is a problem that has not been addressed before. Droplet aerobreakup has mainly been studied in shocktube or wind tunnels facilities, where droplet suddenly experiences a high constant air speed. This is in contrast with the problem studied in this thesis, where droplet are 
initially in a quiescent flow and then the air velocity starts to increase gradually with an acceleration also increasing, until the acceleration and the velocity have reached values that allow for droplet breakup. The problem is then a non-stationary problem and transient effects need to be considered. Accelerating and decelerated non-uniform flow field have only been studied for non-deformable spheres, or droplets that are small enough to neglect deformation and it has proved to be a very complex problem since there still are contradictory results. Therefore, the novelty of this work is to study the aerobreakup of droplets in a non-stationary flow.

The problem of a droplet being approached by an airfoil is of special interest in aerospace applications. In particular, when a plane flies through a cloud, the water droplets inside the cloud will experience this flow field velocity when any lift surface such as the wing of the plane approaches. The interest in studying this problem is that these water droplets, when they are supercooled and impinge on lift surfaces of a plane, can create ice on them changing its aerodynamic lift and drag forces and resulting in a change in the performances of the plane, which in turn could cause accidents and the loss of the plane.

An experimental investigation has been conducted in the rotating arm facility at INTA covering droplets diameter from $0.3 \mathrm{~mm}$ to $3.6 \mathrm{~mm}$. Droplets were generated and allow to fall in the path of an incoming airfoil. Three airfoil sizes (leading edge radius of $0.103 \mathrm{~mm}, 0.070 \mathrm{~mm}$, and $0.029 \mathrm{~mm})$ and five airfoil velocities $(50 \mathrm{~m}=\mathrm{s}$, $60 \mathrm{~m}=\mathrm{s}, 70 \mathrm{~m}=\mathrm{s}, 80 \mathrm{~m}=\mathrm{s}$ and $90 \mathrm{~m}=\mathrm{s}$ ) were used during the test. By means of shadowgraph technique, video images of the deformation and the breakup of the droplets were recorded. The flow field generated by these airfoils was characterized in advance using PIV technique. A tracking software was developed to obtain quantitative data on the droplet deformation and the breakup from the images. During the thesis, first, a characterization of the specific flow field that droplets actually senses when an airfoil is approaching is made and the principal flow parameters involved in the problem of deformation and breakup of droplets are obtained. Then, a data reduction method, the so-called HOSVD, was applied to the problem aiming to provide insight in the underlying physics. Two tensors were constructed: one containing the deformation information and the other containing the breakup time. These tensors were used to extrapolate outside the tensor and to interpolate inside. And finally, the definitions of the onset of the breakup for each mode have been discussed and a breakup criterion equation has been proposed.

It was found that in the most of the cases that were addressed during the experimental campaigns, the breakup type was 'bag and stamen'. In a few cases 'bag' and 'shear' breakup were also identified. Unsteady effects due to unsteady slip velocity and acceleration profiles play a critical role in the droplet deformation and breakup processes. In the cases addressed in this thesis (continuously accelerating flow) the effect was to anticipate significantly the onset of breakup. It was found that if the flow acceleration profile times the square of the droplet residence time is constant, droplet deformation (its instant aspect ratio) depends on the slip velocity only. this suggests that the problem is, at least, governed by a parameter that involves two characteristic times: the characteristic time of the flowfield variation and the droplet residence time.

This thesis has been the first step, only, in a long term development process aiming to generate reliable engineering methods to predict droplet behavior in the vicinity of aircraft wings. This required the availability of reliable databases that can be used for algorithm development purposes. Because of their own nature, the experiments involved are complex and expensive. Then, in this context, it has been found that High Order Singular Value Decomposition is a rather adequate data reduction method for 
this problem. The reason is that it allows for the generation of clean and densified databases (that are obtained after a limited set of experiments) that can readily be used for model development purposes.

A new breakup criterion has been proposed to predict breakup in the "bag and stamen' mode that has prove to be prevailing one in the majority of experimental cases that were addressed in this thesis. Again, the breakup criterion depends on a dimensionless time that is the ratio of the characteristic droplet deformation time to the flow field typical variation time. 


\section{Chapter 1}

\section{INTRODUCTION}

The present thesis will cover the study of droplet deformation and breakup in the vicinity of the leading edge of an airfoil. It consists of six chapters. In this first chapter the problem under study is posed and an introduction is made on the reasons for studying this problem. A literature review on both the basic aspect of droplet breakup and the possible application in aerospace science is presented and finally the objectives are enumerated. Then the second chapter describes the setup of the experiments conducted during this investigation consisting of three experimental campaigns on droplet deformation and breakup due to an approaching airfoil and measurements of the flow field generated by this airfoil. In the third chapter an experimental characterization of flow field that droplets experienced during the tests was made and first conclusions including a scale law on the deformation and breakup of these droplets were obtained. Then, in the forth chapter a reduction method, High Order Singular Value Decomposition (HOSVD), was applied to the problem. And in the fifth chapter a new breakup criterion is proposed for the specific problem of droplets in the vicinity of an incoming airfoil. Finally, the main conclusions are summarized.

\subsection{MOTIVATION}

\subsubsection{Basic Aspects}

The aim of this thesis is to study the problem of the deformation and breakup of a droplet that is immersed in a flow field where the velocity and the acceleration that the droplet senses increases continuously. This is a problem that has not been addressed before. Droplet aerobreakup has mainly been studied in shock-tube or wind tunnels facilities, where droplet suddenly experiences a high constant air speed. This is in contrast with the problem studied in this thesis, where droplet are initially in a quiescent flow and then the air velocity starts to increase gradually with an acceleration also increasing, until the acceleration and the velocity have reached values that allow for droplet breakup. The problem is then a non-stationary problem and transient effects need to be considered. Accelerating and decelerated non-uniform flow field have only been studied for non-deformable spheres, or droplets that are small enough to neglect deformation and it has proved to be a very complex problem since there still are contradictory results. Therefore, the novelty of this work is to study the aerobreakup of droplets in a non-stationary flow. 
The non-stationary flow chosen in this study is the one originated by an airfoil that is approaching a droplet at a constant velocity. The flow field velocity that surrounds an airfoil that is moving at a constant velocity is actually a stationary flow as seen from the airfoil reference of system, which means that at a each position from the airfoil the velocity will remain constant in time. In particular, nearest to the airfoil the velocity is larger, and along the stagnation line the velocity increases as the distance to the airfoil decreases in an exponential way. This allows for the possibility to know exactly the flow field generated by the airfoil by knowing the position relative to the airfoil. However, the fact that the flow field generated by the airfoil is considered stationary respect to the airfoil does not mean that the flow field that surrounds the droplet that is being approached by this airfoil is also stationary since the droplet is not fixed to the model. In fact, the velocity that a droplet in the stagnation line of an airfoil sees as the airfoil approaches at a constant velocity increases with time.

The flow field under study, where droplets are, is considered to be uniform in the vertical direction. If the droplet characteristic length is much smaller than the leading edge curvature radius of the airfoil, which is indeed the case in this study, the droplet senses the airfoil as a flat wall and therefore the velocity can be considered uniform along the vertical direction. In addition, though the droplets actually fall due to the gravity, the vertical fall velocity is small compared to the model velocity, which in practice means that the vertical distance traveled by the droplet is small enough to assume that the droplet remains in the stagnation region.

\subsubsection{Technological Aspects}

The problem of a droplet being approached by an airfoil is of special interest in aerospace applications. In particular, when a plane flies through a cloud, the water droplets inside the cloud will experience this flow field velocity when any lift surface such as the wing of the plane approaches. The interest in studying this problem is that these water droplets, when they are supercooled and impinge on lift surfaces of a plane, can create ice on them changing its aerodynamic lift and drag forces and resulting in a change in the performances of the plane, which in turn could cause accidents and the loss of the plane. Therefore, the problem of the impinging of supercooled droplets on aerodynamic surfaces and the ice formation on them is a really important subject to be studied. For droplets of diameters below $50 \mu \mathrm{m}$, this problem have been widely studied and there is no need to continue studying, however for bigger droplets, which are called 'Supercooled Large Droplets' (SLD), this problem is still under consideration. Observations of droplet of diameters up to $3 \mathrm{~mm}$ have already been reported. In fact SLD, though its conditions are rare, have posed to be a problem during the last decades since the fatal accident at Rosewland in 1994. Because of its larger size, these droplets experience phenomenon such as deformation, which change its shape and consequently its drag, and can also even breakup before impinging. These phenomenon, which are neglected for smaller droplets, need to be studied for these larger droplets in order to be able to determine the amount of droplets that reached the lift surface and its position, which is necessary to study the ice accretion.

The first step to study what happens to supercooled large droplets when a lift surfaces is approaching is to study what happens to water droplets of the same size at air temperature when an airfoil is approaching. And this is the purpose of the present thesis. The present investigation will cover mainly droplet diameters from $0.3 \mathrm{~mm}$ up to $3 \mathrm{~mm}$, which are indeed in the range founded for SLD, and the airfoil velocity will cover from $50 \mathrm{~m} / \mathrm{s}$ up to $90 \mathrm{~m} / \mathrm{s}$. 


\subsection{LITERATURE REVIEW}

\subsubsection{Literature Review on Droplet Deformation and Breakup}

Droplet breakup due to strong aerodynamics forces have been widely studied over the last half-century, starting with the works of Lane [56], Hinze [36], Wolfe and Andersen [102], or Ranger and Nicholls [77, 78], passing through Krezeczkowski [54], Pilch and Erdman [73], O'Rourke and Amsden [67], Wierzba and Takayama [101], Wierzba [100] or Hsiang and Faeth [40, 41] up to the more recent works of Han and Tryggvasson [30, 31], Luxford et al. [24, 60, 59], Chen [14], Theofanous et al [92, 91], Guildenbecher [29] and Theofanous [90].

Attention could be paid to the type of the approach to the problem, either numerical, theoretical or experimental. Some of previous mentioned works were numerical approaches such as those of Han and Tryggvasson [30, 31], and Chen [14] or only theorize [36] while the rest were experimental approaches. Most of the experimental studies have been conducted in shock-tubes $([77,101,40,41])$, or wind tunnels ([56, 24, 60, 59, 100, 54, 92, 91, 90]).

There are many ways to organize a literature review. However, this literature review is presented in a chronological order to give an overview of the problem along its history and the current state of the art. It will include mainly experimental works related to droplet deformation and breakup. However, some numerical works on droplet deformation and breakup and some experimental works related to non-stationary flows will also be included.

The earliest experiments on droplet breakup to be mentioned are those of Lane in 1948-49 [56]. In his first series of experiments he used a small vertical wind tunnel that provided a uniform air stream $u$, where large water droplets of diameters $d$ between 0.5 and $5 \mathrm{~mm}$ fell down the axis and were photographed by the light from a microflash. The air velocity $u$ was increased until breakup was observed and the droplet velocity of the entrained drop $v$ at the instant of breaking was measured. The increasingly flattening of the drop as it came under the influence of the air stream was noticed and the feature of the breakup was described as 'blowing out into the form of a hollow bag attached to a roughly circular rim' (named later as 'bag breakup mode'). The results of this experiment were given in the form of the equation $(u-v)^{2} d=612$, which can be translated to a constant breakup Weber number for all the range tested $W e_{B K}=\rho_{a}(u-v)^{2} d / \sigma=10\left(\rho_{a}\right.$ stands for the air density, $\sigma$ stands for the surface tension, $W e$ stands for the Weber number and the subindex $B K$ denotes breakup). Additional tests with drops of different liquids were carried out. Viscosity appeared to influence the breakup process only when it was very large, tending to retard the drop breakup.

Lane not only made experiments in the wind tunnel, but also in fast air blasts. Following the idea that when the relative velocity between the drop and the air increases above the critical and reaches a certain value larger, the bag breakup mode might be substituted by a different mode of breakup, a blast gun was used to increase this relative velocity up to $100 \mathrm{~m} / \mathrm{s}$. The blast gun was similar in principle to the shock tube which had been used in previous experiments to study the propagation of the shock waves. Another mode of breakup (named later as 'Bag and Stamen') was founded. Lane described and compared it to previous breakup mode as 'it is deformed in the opposite direction and presents a convex surface to the flow of air. The edges of the saucer shape are drawn out into a thin sheet and then into fine filaments which in turn breakup into droplets'. The velocities necessary for the breakup in transient blasts were lower than 
in steady stream, which was explained in terms of Taylor vibrating system analogy. Measurements of the ratio of the lenticular body of the deformed droplet to the original droplet diameter were quite close to the theoretical value of 2 deduced by Taylor.

To obtain more quantitative data and also to increase the air blast velocity, another more powerful blast gun was used obtaining velocities up to $600 \mathrm{~m} / \mathrm{s}$ and another mode of breakup (named later as 'shear stripping'). Lane described it as 'the original spherical drop being distorted into a saucer-shaped disk while a thin layer of liquid is stripped off the drop and rapidly breakup into droplets'. Two mechanisms leading to this breakup were discussed. Lane first suggest that a layer of liquid is stripped off the droplet by the air, being the thickness of that layer calculated by the approximate boundary layer, which seems to be supported by experimental observation only for low velocities. The other suggested mechanism is the production of unstable waves on the surfaces, which seems to be supported by high velocities observations.

This work in the earliest fifties of Lane is very important in the sense that he first covered the three main modes of breakups, he determined some breakup criterion based on the Weber number for the bag breakup, he discussed the two principal mechanism leading to the so-called shear stripping breakup, he made experiments in both wind tunnel and blast gun facilities and he first discussed the transient effects.

Hinze in $\mathbf{1 9 5 5}$ showed theoretically that the critically Weber number depended not only in the viscosity number but also on the way in which relative velocity varies with time [36]. For a true shock exposure, the critically Weber number was smaller (for smaller viscosity numbers, $W e_{B K}=13$ ) than that of the falling drop (for smaller viscosity numbers, $W e_{B K}=22$ ).

A good earlier review regarding breakup times and size distribution of the resultant droplets from the breakup can be found in the report of Wolfe and Andersen [102], in 1964, leading them to state that the breakup of liquids was shown to be a rate process and hence must be considered from a time-dependent approach rather than from purely dimensionless parameters, such as Weber or Bond numbers. They developed a theoretical model of the breakup of liquid drops due to aerodynamic forces, postulating that pressure and frictional drag on the drop were the responsible for the dual breakup mechanisms (bag and sheet stripping) observed. The aerodynamic flow produced a deformation of the drop which in turn produced a sharper curvature at the outer edges of the drop than in the middle of the drop. This curvature produced a higher surface tension pressure at the edges of the drop than in the middle, which opposed the deformation originated by the gas pressure, leading to bag breakup for velocities near the critical velocity required for breakup. For higher velocities, however, the friction stress at the drop edges became larger than the surface-tension pressure making the drop undergo stripping breakup. For intermediate conditions, the characteristics features of both bag and stripping breakup were exhibited. For both the bag and stripping breakup, quantitative expressions were derived to predict the breakup times of liquid drop subjected to aerodynamic flow of a given velocity, $u$ (being original droplet diameter, $d$, surface tension, $\sigma$ and viscosity $\mu$ ).

$$
t=\frac{d}{\left(\sqrt{\left(\frac{16 \mu}{d \rho_{l}}\right)^{2}+\frac{2}{\rho_{l}}\left(\frac{1}{2} \rho_{a} u^{2}-\frac{2 \sigma}{d}\right)}-\frac{16 \mu}{d \rho_{l}}\right.}
$$

A comprehensive, experimental parametric study (relative velocities from 15 to $140 \mathrm{~m} / \mathrm{s}$, surface tension from 0.018 to $0.487 \mathrm{~N} / \mathrm{m}$, liquid densities from 750 to 14000 $\mathrm{Kg} / \mathrm{m}^{3}$, and drops sizes from 500 to $3000 \mu \mathrm{m}$ ) was also conducted on the breakup times of drops of water, mercury and silicons fluids using shock-tube and high speed 
photographic techniques. Experimental breakup times obtained experimentally were compared both to available theories and also to the developed theory, showing very good agreement. Experimental photographs of the breakup process demonstrated the existence of the transition region between the bag and stripping breakup mechanisms that includes characteristics of the both modes.

Another experiments on aerodynamic shattering of liquid drops are those of Ranger and Nicholls published in 1968 [77] and 1972 [78]. In their first study, a stream of water monodisperse drops of diameters $d_{0}$ ranging from 750 to $4400 \mu m$, fall vertically through the air in the test section of an helium driven shock tube. The Mach number of the shock wave $M_{s}$ was in the range from 1.5 and 3.5. Initially the convective flow velocity $U_{r}$ relative to the motionless drop for a $M_{s}=2,0$ was approximately sonic and equal to $430 \mathrm{~m} / \mathrm{s}$. The type of regime studied was the stripping breakup. The shadowgraph technique was employed and a collimated beam of high intensity light was used to back-light the drops. An image converter led to obtain individual photographs, while a rotating drum camera provided streak photographs. The results of the experiments were the drop deformation, drop displacement and breakup times. They found that the breakup could be divided in two stages, the first one comprising the deformation as ellipsoids and the second where surface stripping, produced by the shearing action of the convective flow, rapidly reduced the drops to clouds of micromist. Mach shock waves larger led to maximum deformation attained larger and times to get the same degree of deformation smaller. If the Mach shock wave number was large enough, 1st and 2nd stages of the breakup occurred simultaneously. An interesting finding of Ranger and Nicholls was that the dimensionless breakup time, defined as $\bar{T}=\left(t U_{r} / d_{0}\right)\left(\rho_{G} / \rho_{L}\right)^{0,5}$, seemed little variation and its value was approximately equal to 5. The $\rho_{G}$ and $\rho_{L}$ stood for the gas and liquid velocity respectively, and $t$ stood for time. Finally a boundary layer stripping analysis was conducted to explain the stripping breakup based on the experiments findings.

In their second study published in 1972 [78], only droplets between 1000 and 2700 $\mu m$ were considered, and incident Mach shock wave number was between 1.5 and 5.7, leading to Weber number ranging from 700 to 85500 , convective flow Mach number $M_{2}$ from 0.6 to 1.7 , and Reynolds number from 700 to 41300 . The results of this study included also breakup distance and ultimate atomized particle size. The size of the tiny particles into which a droplet is atomized was found to be in the order of 1-10 $\mu \mathrm{m}$. And a quantitative breakup criterion was given for the time the droplets were completely disintegrated, which was found to be equal to the time the droplet had been accelerated to achieve a speed approximately equal to 60 per cent of the convective flow velocity.

In 1980, Krzeczkowski conducted an experimental study on deformation mechanism and breakup duration of liquid droplets due to an air stream [54]. A wind tunnel, a droplet generator, a photo camera and a spark flash were used. He found four cases of liquid droplet breakup: bag mechanism, bag-jet mechanism, transitional mechanism and shear/stripping-off mechanism. A transition map that can be found in figure 7 in [54] was established relating the conditions of the flow and the deformation mechanism . He also presented data on droplet deformation and breakup for different liquids and finally he concluded that the mechanism of droplet deformation and disintegration as well as break-up duration depended on Laplace number, the viscosity ratio $\mu_{l} / \mu_{g}$ ( $\mu_{l}$ and $\mu_{g}$ stood for liquid and gas viscosity respectively), and specially on the Weber number.

An experimental study regarding non-stationary flow was published by a Temkin and Metha in 1982 [89] for water droplets in the range of 115 to $187 \mu \mathrm{m}$ of diameter. The maximum velocity that droplets were exposed was $10 \mathrm{~m} / \mathrm{s}$ giving a maximum 
Weber number equal to 0.137 . It was explicitly argued by the authors that this range of droplets and velocities was chosen in order to neglect droplet distortion. This shows the difficulty that distortion added to the problem of non-stationary flows. These droplets were exposed to propagating $\mathrm{N}$-waves produced by a conical-driver shock tube to obtain both acceleration and deceleration conditions. Stroboscopic photography was employed to obtain displacement data. An interesting thing was that they used the following non-dimensional acceleration parameter in order to include the non-stationary effects in the drag coefficient:

$$
A_{n}=\left(\frac{\rho_{p}}{\rho_{0}}-1\right) \frac{D}{U_{r}^{2}} \frac{d U_{r}}{d t}
$$

The $\rho_{p}$ and $\rho_{0}$ stood for the droplet and air density respectively, $D$ for the droplet diameter, $U_{r}$ for the relative air velocity and $t$ for time. Their conclusions were that for decelerating flows the unsteady drag was always larger that the steady drag, while for accelerating relative flows the unsteady drag was always smaller than the steady one.

A good review of the mechanisms of acceleration-induced breakup of liquid drops up to $\mathbf{1 9 8 7}$ is the one found in Pilch and Erdman [73]. There, on the time scale of the drop breakup considered, the flow field was assumed constant for all the cases. Under this hypothesis, they postulated that there were five distinct mechanisms of drop breakup as determined by the initial Weber number (descriptions were taken from [73]):

1. Vibrational breakup $(W e \leq 12)$ : The flow field interacts with the drop in such a way as to increase the oscillation amplitude of the oscillations developed at the natural frequency of the drop, which in turn causes the drop to decompose into a few large fragments.Vibrational breakup does not necessarily occur in every instance.

2. Bag breakup $(12<W e \leq 50)$ : A thin hollow bag is blown downstream while it is attached to a more massive toroidal rim. The bag eventually bursts, forming a large number of small fragments; the rim disintegrates a short time later, producing a small number of large fragments.

3. Bag-and-Stamen breakup $(50<W e \leq 100)$ : A column of liquid (stamen) is formed along the drop axis parallel to the approaching flow, while at the same time, as in bag breakup, a thin bag is blown downstream while being anchored to a massive toroidal rim. The bag bursts first and the rim and stamen disintegration follows. This transitional breakup mechanism, that is also called 'multimode breakup', was sometimes referred to as 'club breakup', 'umbrella breakup', 'claviform breakup' or 'parachute breakup'.

4. Sheet Stripping breakup $(100<W e \leq 350)$ : A thin sheet is continuously drawn from the periphery of the deforming drop. The sheet disintegrates a short distance downstream from the drop. A coherent residual drop exists during the entire breakup process.

5. Wave crest stripping followed by Catastrophic breakup (We $>350)$ : At still higher Weber numbers, large-amplitude, small-wavelength waves are formed on the windward surface of the drop. The wave crest are continuously eroded by the action of the flow field over the surface of the drop. This process is referred to as wave crest stripping. Large-amplitude, long-wavelength waves ultimately penetrate the drop creating several large fragments before wave crest striping can be 
significantly reduce the drop mass. Drop penetration by large-amplitude surface waves is referred to as catastrophic breakup. Catastrophic breakup leads to a multistage process in which fragments and fragments of fragments are subject to further breakup. This cascading process continues until all the fragments have Weber numbers below a critical value.

The figure 1 in [73] is a well known figure that schematizes breakup mechanisms just described.

In the same year (1987), O'Rourke and Amsden presented a model on droplet deformation and breakup called the TAB model [67]. This model was based on the analogy, first suggested by Taylor [88], between an spring-mass system and a droplet oscillating. The droplet was divided into two halves and the tension force, the viscous force and the pressure forces were supposed to act through the center of mass of the half droplet. In the spring-mass system the spring force, the damping force and the external force were analogue to the tension force, the viscous force and the pressure forces. Some coefficients were obtained from theoretical considerations and some others from experimental values. A final equation was obtained for the displacement of the equator of the droplet from its initial position. Breakup was supposed to occur when the displacement of the equator was equal to the droplet radius. This model has been well accepted and used to determine the breakup in numerical codes due to its simplicity.

Later in 1988, Wierzba and Takayama [101] re-examined the stripping-type breakup problem by using a new visualization technique: double-exposed pulse laser holographic interferometry. They made experiments in a shock tube with shock wave Mach numbers from 1.3 to 1.5 in atmospheric air. Two sizes of water droplets were examined: diameters of 1030 and $4300 \mu \mathrm{m}$. The Reynolds and Weber number were in the range of 1.38 to $10.4 \times 10^{4}$ and 600 to 7600 , respectively. As a light source, they used a Q-switched ruby laser and parabolic schlieren mirrors. Then an argon-ion laser was used to reconstruct the holograms. They measured the lateral diameter, which was the maximum diameter observed. The lateral diameter $d$ divided into the initial drop diameter $d_{0}$ (the so-called lateral deformation $d / d_{0}$ ) was plotted against the dimensionless time defined as $\bar{t}=\left(t u_{2} / d_{0}\right) \rho_{2} / \rho_{l}$ where the subindex 2 stood for the air properties, $\mathrm{u}$ stood for velocity and $\rho$ for density. They found that larger initial diameter resulted in larger maximum lateral deformation for larger dimensionless times. In particular the maximum lateral deformations for the smaller droplets tested were between 2.4 and 2.9 , and for the bigger droplets round 3.6. By comparing with previous data they found larger discrepancies after the lateral deformation had reached its maximum. Finally, they divided the stripping process in four stages. During the first stage, at the separation point, small lips on the leeward surfaces were formed while the droplet remained spherical. Then, in second stage the leeward surface was getting flattened between the separation points until the appearance of stripping initiated at separation point by boundary layer separation. In the third stage a continuous stripping of microdrops appeared while deformation continued until lateral deformation reached its maximum. Then the remaining parts os the droplets broke up into comparative large numbers. The fourth stage was called 'secondary stripping' and consisted of the stripping of microdrops from the remaining fragments.

In the early nineties (1990), Wierzba [100] published another article which contained a good discussion on the physical aspects and on the work performed up to that date regarding the critical Weber number, usually defined as the minimum Weber number necessary to break up the droplet, in particular in the bag breakup regime. An expe- 
rimental study was conducted on the breakup of droplets for Weber numbers between 10 and 14. He used an small horizontal wind tunnel and droplets of diameters of 2.22, 2.6 and $3.9 \mathrm{~mm}$ generated by vibrating a water jet using the Rayleigh theory. Spark shadowgraph and a high speed camera (frame rate of $5000 \mathrm{fps}$ ) were employed. The transition between vibrational breakup and bag breakup was discussed. The maximum lateral deformation measured was between 1.32 and 1.62, being larger in the regular bag-type breakup.

Another set of experiments are those of Hsiang and Faeth published in 1992 [40]. They made experiments in a shock tube with the driven section open to the atmosphere, similar to that of Ranger and Nicholls [77], that provided test times of 17-21ms. The droplet generation system, based on the the Rayleigh jet vibrating theory, provided a uniformly spaced stream of monodisperse drops between 500 and $1550 \mu \mathrm{m}$, where a system based on charging every other drop and deflecting charged droplets allowed a larger space between drops needed to observe the later stages and outcomes of the bag and multimode breakup. In order to obtain the dynamics of breakup, droplets were observed by means of pulsed shadowgraph pictures and mainly, because of its simplicity, using motion picture shadowgraph within a darkened room. Additionally, a single pulse holography was used to observe the outcome of the breakup. Ranges of the dimensional parameters were: $\rho_{L} / \rho_{G}$ of 580-12000, We of $0.5-1000$, Re of 300-16000 and Oh of 0.0006-4, where Ohnesorge number was limited to less than 0.1 for the experiments leading to the outcome of the breakup. A transitional map We versus Oh for the different breakup regimes was presented in figure 1 in [40], where two additional deformation regimes namely non-oscillatory deformation and oscillatory deformation were added and bag-jet breakup regime was namely multimode. For $\mathrm{Oh}<0.1$, the We transition for each regime was 1.1 for non-oscillatory deformation, 3 for oscillatory deformation, 13 for bag breakup, 35 for multimode breakup, and 80 for shear breakup. These results agreed well with Krzeckowski's results [54]. The We for the onset deformation and breakup regimes increased with increasing Oh, with no breakup observed for $\mathrm{Oh}>4$ due to the stabilizing effect of the viscosity. Regarding times to breakup, an expression was given that depended only on the Oh number for $\mathrm{We}<1000$ :

$$
\frac{t_{b}}{t^{*}}=\frac{5}{\left(1-\frac{O h}{7}\right)}
$$

where $t^{*}=d_{0} \sqrt{\left(\rho_{L} / \rho_{G}\right)} / u_{0}$. This expression was in accordance with Ranger and Nicholls expression for a very small Ohnesorge number. They were aware of the importance of the relaxation velocity due to the droplet drag, which in turns depended on the droplet deformation. An expression was given for the cross diameter $d_{c}$ which depended on the maximum cross diameter $d_{c_{\max }}$ and it was valid before reaching this maximum cross diameter only, which occurred at $t / t^{*}=1,6,30 \%$ of the time to breakup:

$$
\frac{\left(d_{c}-d_{0}\right)}{\left(d_{c}-d_{0}\right)_{\max }}=\frac{1}{1,6}\left(\frac{t}{t^{*}}\right)
$$

Another expression was given for maximum cross diameter $d_{c_{\max }}$ depending on the Weber number:

$$
\frac{d_{c_{\max }}}{d_{0}}=1+0,19 \sqrt{W e}
$$

Another study by the same authors was conducted and published in 1993 [41]. They used the same arrangement as the previous one with the difference that double-pulsed 
holography was used. Liquid droplets were the same as in the previous experiments, water,n-heptane, ethyl alcohol and various glycerol mixtures, but only a diameter of $1000 \mu \mathrm{m}$ was used, leading to the following ranges of the other parameters: $\rho_{L} / \rho_{G}=$ $585-985, \mathrm{Oh}=0.0025-0.039, \mathrm{We}=15-600$, and $\mathrm{Re}=1060-15080$. Experimentally, the Eötvös number $E o=\frac{\rho_{L} d^{2}}{\sigma} \frac{\mathrm{d} u}{\mathrm{~d} t}$ at the end of shear breakup was found to be nearly constant and independent of Weber number $W e$ (based on initial conditions), $E o_{c r}=$ 16.

Regarding non-stationary flows, a good review on drag coefficient of either spheres or droplets in these kind of flows was included in the article by Igra and Takayama published in the same year (1993) [44]. They also conducted shock tubes measurements on spheres of Polystyrene (droplets diameter of 3.2, 4 and $4.8 \mathrm{~mm}$ ), Nylon (droplet diameter of 3.2 and $4.0 \mathrm{~mm}$ ) and Polyamide (diameter of $0.5 \mathrm{~mm}$ ). The measurements were taken using holographic interferometry technique. The incident shock wave number were $1.27,1.5$ and 1.8 and the Reynolds number varied between $6 \times 10^{3}$ and $10^{5}$. They concluded that the drag coefficient of a sphere in a non-stationary flow could be much larger than in a steady flow, being the difference up to $50 \%$ for Reynolds number between $10^{4}$ and $10^{5}$, and even more than $100 \%$ for Reynolds lower than $10^{4}$.

A Droplet Deformation and Breakup of droplets model (DDB model), similar to the TAB model was presented by Ibrahim et al in the same year (1993) [43]. DDB model assumed that the liquid drop was deformed due to a pure extensional flow from an initial spherical shape into an oblate spheroid. Drop dynamics were formulated in terms of the motion of the center of mass of the half-drop. The problem was addressed by the energy equation, with no heat exchange with its surroundings. The internal energy came from the kinetic and the potential energy due to the surface tension of the droplet. The work was done by pressure and viscous forces. It seemed to work better than the TAB model and was widely used in numerical simulations. However, there was a mistake in the results, as explained in [58], and the TAB model was actually better in the case presented.

Two articles that studied the droplet breakup of axisymmetric liquid drops in a numerical way were those of Han and Tryggvason in 1999 [30] and 2001 [31]. They examined two density ratios (droplet density $\rho_{d}$ divided by the density of the surrounded field $\left.\rho_{0}\right) 1.15$ and 10 and studied the influence of the main non-dimensional parameters such as the Onhesorge number (Oh), Eotvos number (Eo), density ratio and viscosity ratio in the way the droplet breakup up and its critical Weber number. In their first work, they studied the breakup due to acceleration by a constant body force, such as the gravity force. They founded both the backward-facing bag breakup and the forward-facing bag breakup and they concluded that for low Ohnesorge number, the governing parameters were the Eotvos number and the density ratio, while for the larger Ohnesorge numbers, the effect of the viscosity reduced the deformation rate. In their second work in 2001, droplets were under an impulsive acceleration. For this kind of acceleration, the backward-facing bag breakup was only seen for the largest density ratio and for Weber number larger than 10 . Then, as the Weber number increased, the forward-facing bag breakup was also observed.

Probably one of the most interesting experimental work up to now was the one of Theofanous et al in 2004 [92] because of its discussion. For their experiment, they used the Alpha facility, which was a supersonic wind tunnel especially designed to study the aerobreakup of the viscoelastic fluids. It consisted of two receiver tanks, a supply tank connected via a vertical transparent test section of diameter 101,6 mm where the flow was accelerated to supersonic speeds by a converging-diverging nozzle. 
They operated at Mach 3 and at pressure levels from 0.01 atm down to $0.0001 \mathrm{~atm}$. They were able to generate droplets from $2 \mathrm{~mm}$ up to $20 \mathrm{~mm}$ that reached the nozzles in perfect stable spherical shape. A photocell detected that the drop had reached a certain position and a diaphragm was broken and the monitoring system, consisting of a high-speed camera and a synchronizer copper-vapor laser, was activated. They used droplets of diameter around $4 \mathrm{~mm}$ of two fluids: Tributyl-Phosphate (TBC) (density: $0.978 \mathrm{~g} \mathrm{~cm}^{-3}$; viscosity: $4 \mathrm{mPa} \mathrm{s}$ ) and Gliceryn (density: $1.26 \mathrm{~g} \mathrm{~cm}^{-3}$; viscosity: 13 $\mathrm{mPa}$ ). Reynolds number ranged from 200 to 31600, while the Weber number varied from 10 to 2000 . For the TBC droplets they found the following regimes:

- Pulling (We <26).

- Bag and MultiBag $(26<\mathrm{We}<44)$.

- Piercing $(44<\mathrm{We}<1000)$.

- Stripping (We >1000).

The 'pulling' regime and 'multibag' regimes were new, which they thought was because in previous studies, droplets were smaller and there were not enough resolution to interpret the results. Piercing appeared to be pure, without effects of shear/stripping, starting from one wave (the bag) and then increasing as the Weber number increased. The order of magnitude where piercing was found was several order smaller than previous experiments. For the Glycerin droplets similar observations were found for bigger droplets except that the pulling regime disappeared due to the higher viscosity. They found deformation up to Weber number of 113, 'Bag' for $116<$ We $<2000$, and 'Piercing' for Weber on the order of 2000. Then, there was a good discussion on the piercing mechanism, which seemed to be caused by the Rayleigh-Taylor instabilities. The number of waves that would fit on a 'disc' of diameter $d_{\max }$ was expressed as follows:

$$
n=\frac{2 d_{\max }}{\lambda_{d}}=\frac{1}{2 \pi}\left(C_{D} \Phi_{1}\right)^{1 / 2} \Phi_{2} W e^{1 / 2}
$$

The fastest-growing wave $\lambda_{d}$ was obtained from the classic inviscid analysis neglecting the gas density relative to that of the liquid. The $\Phi_{1}$ and $\Phi_{2}$ accounted for the effects of deformation on $C_{D}$ and cross-sectional area respectively. The number of waves was represented against Weber number, and compared to experimental observation and they found good agreement. They also discussed the appearance of the 'pulling' regime and they thought that the limit between the pulling regime and a single wave piercing corresponds to the balance between the viscous-drag-induced motion and the tendency to penetrate. The 'piercing' regime was then clearly appreciated, which they thought the supersonic velocities to be responsible. They argued that the number of waves was increased in a factor of two. Finally they also discussed the stripping regime where shear increased.

A primary discussion on the transient effects on droplet distortion and breakup in relation to aerospace application (in particular to SLD) was found in the work presented by Luxford et al. [24] in 2004. By a simple analysis they led to the conclusion that the main parameter in relation to transient effect was the ratio between the droplet diameter and the airfoil leading edge radius. And they studied the problem of droplet distortion in the vicinity of cylinders by simulations. Then they conducted experiments in a small open-circuit convergent wind tunnel, which was designed to give a linearly increasing 
Weber number with time. They measured the acceleration of some droplets and inferred the drag coefficient, but regarding distortion, only a few droplets of volume equivalent diameter of $350 \mu \mathrm{m}$ at velocity of $25 \mathrm{~m} / \mathrm{s}$ were shown.

Later in 2005, Luxford et al. [60] presented another work on the droplet distortion and drag coefficient at ambient temperature of droplets smaller than $1 \mathrm{~mm}$, at Reynold numbers below 1000 and Weber number higher than 10, in the context of supercooled drizzle droplets. They modeled the drag coefficient as an interpolation between the sphere drag coefficient and a disk drag coefficient being the interpolation factor a function against the Bond number. They discussed the influence of the droplet deformation, which was supposed to deform as two oblate semi-spheroids, on the drag coefficient and they conducted experimental measurements to validate their theory. They used a convergent wind tunnel and they generated the droplets by a continuous jet impinging on a spinning slotted disk in order to prevent coalescence. An improved high-intensity LED strobe was developed to illuminate from the back and high-speed videos and photographs were taken. The experimental and predicted droplet drag were compared showing the substantial effect of droplet distortion on the drag properties.

Another quite comprehensive article regarding non-stationary flows was the one of Jourdan et al in 2007 [46], where an experimental study on the drag coefficient of spheres in a multi-phase variable inclination shock tube was conducted. They used spheres of diameter between $0.29 \mathrm{~mm}$ and $6.5 \mathrm{~mm}$ made of polystyrene, polymer ATECA, polypropylene and nylon. Each spherical particle was suspended in mid-air by a fine filament (spider web), and it was considered that it did not influence the measurements. The incident shock wave Mach number was between 1.1 and 5, allowing for both subsonic and supersonic flows. Shadowgraph images illuminated by an stroboscopic were recorded by a high-speed camera (15000 fps). They compared their data with previous experimental researches and they concluded that the acceleration parameter defined by Temkin and Metha [89] was not a characteristic parameter for the non-stationary flows considered in their study. Although the conditions were somehow different, this is contrast with the conclusions of previous researches such as the work of Igra and Takayama [44]. This shows that this field is still an active field to research and that open questions still exists.

In 2008, Chen [14] conducted a simulation of entire evolution of the stripping breakup by using a multiphase flow solver with a five-equation model of water droplets of diameters $6.8 \mathrm{~mm}$ and $4.8 \mathrm{~mm}$ and Mach number of 1.3 and 1.47. The conditions were chosen to compare to previous experimental result. The aim of the work was to shed light in the process involved leading to breakup, since different theories had been made depending mostly on the visualization technique. They tried to explain the tip formation mechanism by showing the pressure and velocity contour of the droplet. It was found that the high-pressure zones were at front and rear sides of the droplet, while the low pressure zones were at the upper and lower sides. The droplet was compressed by the high pressure zones and elongated in the low pressure zones, which was explained by the pressure field. Inside the droplet, there was also an unbalanced pressure distribution that contributed to the shape deformation. The tip formation at upper and lower sides appeared to be related to the low-pressure zones. Flow instabilities were observed and they concluded that the vortex shedding observed was caused by Kelvin-Helmholtz instability.

In the same year (2008), Theofanous and Li [91] published a study on the shear (previous called stripping) breakup regime, trying to elucidate the mechanism involved by new visualizations based on laser-induced fluorescence (LIF) in combination to 15 ns exposure times. They used droplets of tributylphosphate (TBP) and $3.5 \mathrm{~mm}$ and a 
pulse supersonic wind tunnel that provided a steady flow of duration up to $100 \mathrm{~ms}$, at Mach 3, dynamic pressure up to $10^{5}$ and Weber numbers up to 30000 . They also employed shadowgraph imaging for comparison purpose. Video records were made by two high speed cameras. They found that for Weber larger than 1000, the mechanism leading to the breakup is the shear-induced entrainment (SIE), which contradicted previous hypothesis of a stripping mechanism. For the Weber number between 10 and 100 , they affirmed to be Rayleigh-Taylor piercing, including 'bag' and 'bag and stamen' breakup in subsonic and 'multiwave' penetration in rarefied supersonic flows, which led to the aerobreakup of droplets.

They compared this data with previous data and discussed all the differences and the probable causes that led to previous hypothesis, which they blamed the difference on pressure, subsonic against supersonic, and the lack of quality images to be responsible for the previous thoughts. They explained why the 'multiwave' regime did not appeared before in subsonic but there appeared 'bag' and 'bag and stamen'. They also explained why the catastrophic regime did not really exist, but it was instead a mirage of the shadowgraphs that evidenced the waves. This article is very good article because of the discussion on previous results in the light of the new results.

A very complete review in $\mathbf{2 0 0 9}$ of all the journal articles published regarding droplets aerobreakup is the review of Guildenbecher [29]. Both experimental and simulations were reviewed and used for the discussion. Regarding experimental works, special attention was paid in the first place on the experimental facility used and it was considered four types of facilities:

- Shock tube: The main advantage was that it provided a step change in ambient flow uniform over its surface. However it has low data rate (due to the need to reset the experiment after every run) and limited range of operating conditions.

- Continuous jet: As an alternative to the shock tube it was a very simple facility and had the ability to operate continuously, however drop deformation and breakup should occur in the jet's uniform velocity regime.

- Drop tower: It showed the breakup of droplets when they were accelerated more slowly by a constant body force, the gravity.

- Hybrid methods: It included a mixture of the previous methods

The influence of the flow field variation time was introduced by comparing the drop tower facility and shock tubes to explain the differences between the critical Weber number encountered in the previous literature. Then, attention was paid to the breakup morphology and a similar classification of breakup mode as the one found in Pilch and Erdman [73] was established:

- Vibrational breakup

- Bag Breakup

- Multimode (Bag and Stamen)

- Sheet Stripping (Sheet Thinning)

- Catastrophic Breakup 
Some comment was made on the work of Theofanous et al [92], and the deviation in the breakup morphology for rarefied, supersonic ambient flows. Dimensionless groups were defined and transitional Weber number between the breakup modes were reviewed for both low and large Ohnesorge numbers. For each breakup mode, the physical mechanism leading to each mode of breakup was discussed at length. Traditionally, breakup times used in the literature were defined and the main expression to obtain these for newtonian and non-newtonian drops were reviewed. Finally efforts were made to review and compare the main breakup models for each breakup mode such as the Taylor Analogy Breakup (TAB), the droplet and deformation model (DDB) or the instabilities leading to breakup (K-H Instability). Also some comments were made on the numerical simulation models.

Another droplet breakup criterion, based on analytical considerations, was proposed by Sher and Sher in 2011 [82]. They assumed a symmetrical breakup process where the surface energy of the distorted droplet transformed into the surface energy of $n$ children droplets surface energy while dissipating an energy estimated by Bird et al [8]. The initial critical Weber number for which breakup occurs was obtained by the following expression

$$
W e_{0, c r}=11,93 \sqrt{n^{1 / 3}-1}+30,51\left(C_{D} \sqrt{\frac{\rho_{g}}{\rho_{l}}} O h\right)^{2 / 3}
$$

where $O h$ stands for the Ohnesorge number, $C_{D}$ for the original constant average coefficient, and $\rho$ for the density of either the gas $(g)$ or the liquid $(l)$.

One of the last comprehensive review on the current status of the physics of aerobreakup can be found in the recent article (2011) published by Theofanous [90]. Since the previous review of Guildenbecher [29] summarized all the previous work, the focus of this review was to present what they thought it was correct. They though that previous discrepancies was due to the visualization techniques and they though that the main task was to obtain spatial resolution above $100 \mathrm{pix} / \mathrm{mm}$, and exposure times of a few nanoseconds, specially in the shear regime. In particular, past work mistook the socreated blurry envelop as a measure of flattening, preventing from recognizing the entrained portion of mass in the shadowgraphs. Therefore, new experiments fulfilling visualization requirements were conducted using laser-induced fluorescence, which was used in his previous research [91]. They used the ASOS shock-tube facility obtaining shock Mach numbers up to 3.5, and the fluids employed were water, tributhylphosphate (TBP), glycerol and a wide range of silicon oils of different viscosities.

In this article, the author discussed at length (based, mostly, on experimental results) all physical aspects related to the deformation and breakup of droplets in a gas stream. Specifically, it covered his personal work [71, 92, 91, 90] and the work of many other researchers. Attending to the mechanism leading to the breakup it was established another classification defining two principal breakup regimes:

- Rayleigh-Taylor Piercing (RTP): 'The gas finds its way through the liquid mass, causing gross disintegration'.

- Shear-Induced Entrainment (SIE): 'The gas goes around to induce, through shear, a surface-layer peeling and ejection action'.

The article started with drop deformation. Drop flattening seemed to be important mainly in the RTP regime, because the time to breakup in this regime was provided by the timing for deformation and it set the stage for the first critically. In contrast, in the 
SIE regime, though the remaining coherent drop underwent deformation, the entrainment started very early and the deformation process was not essential in the breakup mechanism. Then the article continued discussing the two main mechanisms leading to breakup: Rayleigh-Taylor piercing and shear-induced entrainment. As stated by him, elementary considerations suggested that the interface in the vicinity of the forward stagnation point was subject to Rayleigh-Taylor (RT) instability, whereas further away, towards the equator, Kelvin-Helmholtz instability became increasingly important. The propensity for RT instability was affected by the interfacial curvature and the acceleration while the deformation alters the shear-force patterns at the interface, and the mean flow development in the outer liquid layers, which further coupled to the interfacial roughness induced by the Kelvin-Helmholtz instability [53, 90]. RTP was the first mayor criticality and with increasing dynamic pressure (or Weber number), SIE is the second major criticality [90]. Additionally, in this article, two graphs were provided that determine the critical Weber number as a function of Ohnesorge number for both breakup regimes (RTP or SIE). Critical Weber number are defined from stream air velocity (uniform gas flow velocity behind the shock wave) and initial diameter (where the droplet was spherical).

One of the most recent articles related to drop deformation and breakup is the one of Kékesi et at in 2014 [49]. This article is of a numerical nature and the Volume of Fluid (VOF) method was applied to study bag and shear breakup regimes induced by steady disturbances. A parametric study was conducted applying different material properties (density ratios of liquid to gas of 20,40,60 and 80 and viscosities ratios in the range of 0.5 to 50) and a wide range of drop Reynolds numbers (between 20 and 200). For a constant Weber number of 20 and varying the rest of parameters, new breakup modes apart from the classical bag and shear breakup were found. In particular they identify five new intermediate breakup modes (as described by them):

- Jellyfish shear: After reaching the spherical cap shape, the edge of the cap moves downstream, stretching the droplet in the streamwise direction. The stretched droplet part approaches the central axis and closes the aft surface, flattens out and returns to an oblate spheroid form. This procedure was repeated once more and then the droplet experiences shear breakup

- Thick rim shear: Having obtained a spherical cap shape, a small bag grows in the middle of the drop and separates as a core droplet that undergoes shear breakup, leaving a thick rim around

- Thick rim bag: Similarly to the thick rim shear, but the core drop undergoes bag breakup

- Rim shear: After reaching the spherical cap shape, the droplet starts to expand in the radial direction normal to the flow maintaining its shape. Thereafter, the rim becomes more distinguishable and breaks from the membrane

- Mixed: After reaching the spherical cap shape and expanding in the radial direction a bag grows. However, instead of bag rupturing the rim moves downstream as compared to the bag and breakups up with shear breakup

For the bag breakup cases the time to reach breakup was considerable larger than the cases where droplets experiences shear breakup, which was postulated to be due to the time needed for the bag itself to develop. A criterion for bag or shear breakup was presenting by comparing the characteristics times 


$$
\frac{t_{\text {shear }}}{t_{\text {bag }}}=A \sqrt{\frac{1}{R e_{g}}}
$$

where $A=1,74 \frac{\mu_{l}}{\mu g} \sqrt{\frac{\rho_{g}}{\rho_{l}}}$ was a constant non-dimensional that contained all material properties and $R e_{g}$ stood for the drop Reynolds number. Shear breakup was expected if the characteristic time for the shear breakup was smaller than for bag breakup. A new regime map was obtained using this criterion that seemed to fit well with the data. Finally, they explicitly argued in their conclusion section that further development should be achieved by accounting for accelerations terms rather than investigating stationary drops as were presented there.

Finally, one of the last approach to the drop deformation and breakup problem is the one published by Sichani and Emani in 2015 [84], which is again a numerical approach. They proposed a new model based on the virtual work principle and potential flow solution. In order to simplified the model they only considered moderate Reynolds number near 1000 and high liquid-gas density ratio $\rho_{l} / \rho_{g}>>1$. They were capable of tracking the drop deformation and distortion up to the onset of the bag rupture in the bag breakup regime. To this end, they modeled the bag dynamics formulation with a two degree of freedom system. They modeled the geometry of the system as two basics geometric shapes attached together: an incomplete toroid (that played the role of the outer rim) and a spherical layer (which served as the bag or the middle part of the system depending on the stage of breakup). They assumed that breakup occurs when the thickness of the bag film reaches a critical value, in particular when this thickness is 0.0075 times the initial droplet diameter, and this was used as the breakup criterion. The models were examined in conditions that were more matched with that of shocktubes or wind tunnel experiments, and these kind of experiments were used to validate the model, introducing a tuning factor in the model. Overall, a good agreement was found between the computed results and the experiments. It appeared that the model was also useful to predict the critical Weber number, being the relative error round $20 \%$ as compared to experimental data. The tuned model predicted well the breakup time for near-critical Weber numbers. However, it increasingly underestimated the onset of breakup time for higher Weber number. They concluded that further studies needed to be conducted to better understood the physical mechanisms leading to the breakup.

\subsubsection{Literature Review on Technological Application Aspects}

A collision of a supercooled-water droplet on an object creates ice on its surface. The ice accretion can cause severe damage and disasters. Icing can cause a problem on the surfaces of ships, power cables, trees, road signs, architectures, and also in aircrafts [27]. Aircraft icing is observed both on the ground and in-flight, being in-flight icing actually a relevant technical aspect that aircraft manufactures need to deal with when assessing aircraft operation. A rather comprehensive review of the in-flight icing phenomenon, its relevance, and the methods for its detection and identification can be found in the article published by Caliskan and Hajiyev [11].

The problem of in-flight icing is the significantly change in the flight performance, stability and control that at the end can result in aircraft failure. Excluding military, private, and charter aircraft, an accident survey of 2062 aviation accidents from 1999 to 2010 showed the importance of the weather related accidents that occupied $7 \%$ among other causes [11]. Ice accretion during in-flight may occur on different parts of the 
aircraft: the leading edge of wing, control surfaces, horizontal and vertical stabilizers, fuselage nose, landing gear doors, engine intakes, fuselage air data ports and sensor and drain system outputs. However, among all these causes, most accidents occur as a result of ice accretion on wing surfaces due to the alteration of the shape of these aerodynamic surfaces that affect the performance and stability of the aircraft[11].

General aviation, commuter aircraft and small transport aircraft are more sensitive to the adverse effects of icing than large commercial transport aircraft because of their size [27]. Ice accretion on aerodynamic surfaces increases the profile drag and reduces maximum lift and stall angle of attack. During take-off, iced aircraft would have an increased ground roll, decreased stall margins and climb rate. Iced wings and tail surfaces are particularly hazardous during take-off since they could lead to serious stability and control problems resulting in the loss of the aircraft, as happened in a number of incidents. During cruise, iced aircraft suffer from a decreased maximum speed capability and efficiency, higher fuel consumption, change in trim characteristics and reduced ceiling height. During approach or landing, the combination of ice on the horizontal tailplane and extended flaps may cause the tailplane to stall, leading to uncommanded 'pitch-overs' for some aircraft.

When a wing leading edge or some other forward-facing component of the aircraft passes through cloud containing supercooled droplet, the impinging droplets release their heat latent and freeze to form ice accretion. The size, shape and location of the ice accretion that will form and its rate and amount, according to [39], depends on:

- The environmental parameters: droplet-size distribution, cloud liquid water content (LWC), median volumetric diameter (MVD), ambient air temperature, pressure and relative humidity and air/water surface tension.

- The aircraft surface conditions: roughness and surface temperature.

- The flow parameters: flight velocity, angle of attack, and icing time.

Some authors, such as [27] consider the ice accretion phenomenon in two distinct parts. The first part is the rate at which the water droplets are intercepted by the body which includes droplet trajectories and impact location and the second part is the rate at which ice is formed.

The first stage in any icing analysis is to calculate what is known as the watercollection or catch-efficiency distribution, which determines where and at what rate the cloud water droplets are deposited on the surface of the body under investigation. Due to the forward velocity of the aircraft, supercooled droplets impinge on the surface of the aircraft when the aircraft flies through a supercooled cloud. The trajectory that a droplet follows, and therefore the location at which it will impact the surface, depends mostly on the droplet size, as the trajectory is determined mostly by the drag force on the droplet [39], which in turn depends on its shape and size. The ratio of the inertia to the aerodynamic forces determines whether a droplet impacts the surface or is swept past the body in the airflow. Larger droplets tend to deviate more from the streamlines having less curved trajectories and then impinging on the airfoil [57]. Moreover, if the droplets are larger enough, they actually breakup before impinging and this changes everything.

The diameter of the droplets within any single cloud will vary. The median volumetric diameter (MVD) of the cloud, which is the diameter above and below which half the mass (or volume) of water is contained, is used frequently to characterize the spectrum of droplets that exists in practice in a cloud. Supercooling is difficult to maintain 
in large droplets at low temperature, therefore, in general, there will be a tendency for the diameter of the supercooled droplets to reduce as the ambient temperature reduces.

The trajectory calculation can be done mainly in two ways:

- Lagrangian: the trajectory of an individual water droplet is tracked from upstream of the body starting in a specific point (usually five to seven chord lengths ahead of the body). This is the most frequently adopted method.

- Eulerian: the volume fraction is computed at the same node positions at which the aerodynamic parameters are known. This approach has been more recently adopted.

For both methods, either a flow solution or a means of calculating the aerodynamic flow is necessary [27].

The rate at which the impinging water will freeze is the second part in which ice accretion could be divided. The heat transfer from the surface of the body, which includes kinetic heating, convective cooling, evaporative cooling, latent heat of freezing and a number of smaller contributions from sensible heating and cooling mechanisms, primarily governs the ice accretion [27]. It is the surface temperature of the body together with the catch efficiency and the size of the incoming supercooled droplets and its temperature which dictates whether accretion is possible and the rate at which this accretion proceeds. Generally, two types of ice shapes may be observed on airfoils $[27,57,39]$ :

- Rime-Ice Accretion: The water droplets freezes fully immediately upon impact on the leading edge and form a dry, opaque and milky-white smooth and streamline ice shape around the leading edge. It usually occurs at low airspeeds, lower temperatures and lower liquid water contents.

- Glaze-Ice Accretion: Only part of the water in the droplets freezes upon impact. It usually forms at temperatures just below freezing point and high liquid water content. It often runs back upward and downward from the stagnation point resulting in a horn-like transparent ice shape.

Dry ice crystals do generally not adhere to the aircraft surfaces after impact, so they are not considered an icing hazard. Icing normally results from the impact of supercooled water droplets. However, if the ice crystals are partial melted or the aircraft surface is wet due to de-icing or the recent penetration of a high liquid water content region, and thus the ice crystal are wet, then the impinging crystals will stick [34].

In 1984 Hansman warned the great importance of the droplet size in aircraft icing, concluding in [33] that the shape of the droplet size distribution function, particularly for larger droplets, was a very important factor and that even a small liquid water content could be a significant icing threat if it consisted of large drops. Temperature, liquid water content and droplet size distribution was found to have an extremely influence in the severity of aircraft icing, particularly near the transition between rime and mixed icing. Additional hazard is due to the presence of large droplets within the cloud distribution that can result in increased impingement limits. The limit of droplet impingement on an aircraft component increases with droplet size. In 1990, Hasman [34] warned again that the presence of significant numbers of droplets in excess of $40 \mu \mathrm{m}$ can therefore result in ice accretions occurring behind the protected regions of the aircraft. 
The certification envelopes defined by the JAR/FAR Appendix C only accounts for icing envelopes comprising water droplets up to diameters of $50 \mu \mathrm{m}$. However, there exists icing clouds containing larger super-cooled droplets, namely as Supercooled Large Droplet (SLD), which can be either freezing drizzle, in the range of 50-400 $\mu \mathrm{m}$, or freezing rain, with droplet diameters beyond $400 \mu \mathrm{m}$. Freezing rain differs from freezing drizzle in terms of the diameter of the droplets and the processes by which the droplets form, as explained by Gent [27].

Actually, droplet sizes up to $3000 \mu \mathrm{m}$ were measured in icing clouds by measurements in-flight conducted by the Federal Aviation Administration, National Aeronautics and Space Administration Glenn Research Center, Meteorological Service of Canada, and the National Research Council of Canada [16]. These super-cooled larger droplets (SLD) have proved to be an icing hazard since the fatal accident of Roseland in 1994 and a new appendix in the FAA regulations is planned to be included in order to account for SLD problem.

The problem of these large droplets is that they do not behave in the same way as the smaller ones. When these large droplets are exposed to the aerodynamic forces induced by the incoming airfoil, they deform and can breakup due to their relatively weaker surface tensions forces and greater surface areas to interact with the external forces. Also splashing can generate droplets that re-impinge in wing zones not protected. Drop deformation modify drop drag coefficient and consequently their trajectory and final impact on the airfoil, and breakup changes drastically the size of the droplets impinging on the surface. Both droplets size and impact location are parameters accounted in ice accretion simulation. Therefore, due to the larger size, the following phenomena that are disregarded for normal droplets $(50 \mu \mathrm{m})$ are not readily disregarded for SLD because of their size:

- Deformation: Droplets initially undergo a transformation from spherical to disk shapes. This distortion results in a change in the droplet drag, which in turn modify the impinging point.

- Breakup: A large droplet approaching a stagnation region will attain breakup condition earlier than a smaller droplet. This is due to the greater inertia of the larger droplet, which leads to a greater velocity relative to the surrounding air. The mechanism of breakup then depends on the prevailing velocity gradients [87].

- Splashing and Rebound: The problem of the splash droplets is that they may reimpinge on aft surfaces causing ice accretion in areas not normally associated with anti- or de-icing requirements. The factors that can affect the splash mechanism include droplet impact velocity, impact angle, droplet size, viscosity, surface tension and surface roughness [87].

A good paper describing the problems of modeling with these supercooled large droplets (SLD) where droplet sizes are greater than FAR/JAR25 Appendix C sizes is the one of Tan and Papadakis [87]. This paper reviews past and present researches on droplet dynamics relevant to SLD ice accretion modeling, and suggest correlations that can account for the first order of magnitude effects. They claimed the lack of understanding in areas such as droplet splashing and filming, increased drag due to changes in the droplet shape or droplet break-up to be the responsible for the inaccuracy in the simulation of existing icing. 
The trajectories of large droplets are relatively unaffected by the viscous forces, due to their greater momentum, therefore they can impinge much further aft than smaller droplets. These characteristic behaviors of large droplets have presented major challenges to icing tests facilities and current generation of ice accretion codes due the lack of experimental data needed to develop the accurate simulation models as stated by Tan in 2005 [86].

There exist studies that conclude that accounting for droplet breakup, splashing and rebound make significant improvement to the simulation of the droplet impingement distribution compared to those without. An example of these studies is again the one of Tan and Papadakis in 2005 [86]. In this study, a droplet breakup, splashing and rebound model was used to simulate the droplet impingement distribution in a clean MS(1)-0317, GLC-305 airfoil, and an 'iced' NACA23012 (with 22 min simulated ice shape) airfoil. They used a breakup model based on the Taylor Analogy Breakup (TAB) model [67], which assumes that droplet oscillatory motion is analogous to that of a damped spring-mass system under-going a forced oscillatory motion. Droplet breakup is then assumed to occur when the displacement of the equator of the droplet from its equilibrium position exceeds half the radius of the undistorted droplet according to O'Rouke and Amsden [67]. They validated the model using experimental data of tests conducted in a horizontal tunnel by Wierzba [100] and in a vertical tunnel by Kennedy and Roberts [50]. In the Wierzba experiments, water droplets of 2220, 2600 and 3900 $\mu \mathrm{m}$ were released from the top wall of the test section where a flare inlet bell-mouth was used to ensure a smooth airflow entering the test section. This is in contrast to the actual in-flight situation of droplets near the leading edge of an incoming airfoil, where the velocity experienced by the droplets is continuously increasing. On the other hand, the experiments conducted on the vertical tunnel consisted of studying droplet breakup beteween 500 and $1991 \mu \mathrm{m}$ at the throat of the tunnel contraction. The velocity distribution in the contraction was designed to simulate the acceleration of the airflow near jet engine nacelles for a range of throat velocities between 45 and $128 \mathrm{~m} / \mathrm{s}$.

Another numerical study by Hospers and Hoeijmakers in 2011 confirms the importance of these phenomenon [39]. The catching efficiency for larger droplets (SLD) is over predicted using the Eulerian method developed by them to calculate ice-accretions on two dimensional airfoils. Therefore, they include in their codes an splashing and a rebound model. The inclusion of a splashing model accounted for a decrease in the catching efficiency on the airfoil, bringing the catching efficiency prediction closer to experimental results. A further improvement was achieved by including a droplet rebound model. However the experimentally and numerically calculated catching efficiencies still differed, which they concluded to be possibly caused by effects due to breakup or aerodynamic effects.

As described by Bragg in his article [10], the size and shape of the ice accretion on unprotected aerodynamic surfaces depend primarily on airspeed, temperature, water droplet size, liquid water content, and the period of time the aircraft has operated in the icing condition. Under a normal icing encounter, when the deicing system is activated, almost all of the ice will be removed since most of the ice accretion would occur over the active portion of the wing deicing system. However, this is not the case in the presence of super-cooled large droplets (SLD). Due to their larger size, the droplets can impinge and accrete as ice downstream of the deicing system. A ridge ice accretion can occur on the aircraft wing behind the leading edge deicing system when the deicing system is activated. In the literature, this type of accretion has been referred to as a spanwise-step ice accretion, a SLD ice accretion, and a large-droplet ice accretion. All refer to a ridge, usually forming aft of the protected region. In 1996, Bragg [9] reviewed 
the aerodynamic effects of the ridge ice accretion and showed that the ridge accretion not only degraded lift and drag, but also adversely affected the aileron hinge moment. This was thought to be the result of a large separation bubble that formed downstream of the accretion, which severely altered the pressure distribution over the aileron. A later study [10] in 2000 led to the same conclusion.

An ice ridge can lead to reduced lift, increased drag, change in moments and a loss in control effectiveness. In addition, the existing ice protection system in the most common aircraft uses engine hot air. This contributes to a decrease in the engine performance. With the use of bleed hot air, fuel consumption is increasing, and maximum engine power is decreasing. Additional detailed studies focused on the influence of icing in aircraft stability, performance degradation, dynamic parameters, and stall conditions have been reported by Lampton and Valasek [55], Han and Palacios [32], Dong and Ai [20] and Campbell et al. [12] respectively.

Regarding the methodology used to study this phenomenon, it could be observed that many studies dealing with this problem are of a numerical nature. In this context, a number of numerical (CFD) approaches have been recently published by Aliaga et al. [3], Ghenai and Lin [28], Hasanzadeh et al. [35], Honsek et al. [38], Iuliano et al. [45], Jung and Myong [47], Nakakita et al. [64], Rendall and Allen [80], and Zeppetelli and Habashi [103].

In practice, these numerical approaches involve an extremely high degree of modeling complexity since multiphase compressible turbulent flows with changing geometries (the ice accretion effect) are considered. Therefore, it is customary to relax some of the model hypothesis so as to generate a software package or an algorithm that can be used for practical prediction purposes.

Among the large number of hypothesis that make up these ice accretion models, one of the most difficult to deal with is the one related to the motion, deformation, and eventual breakup of the water droplets before they impinge on the airfoil/wing.

The effect caused by these hypothesis is even more important when super-cooled large droplets, are considered, as we have previously discussed. For example, Aliaga et al. [3] modeled droplet behavior as a dilute gas-particle system in which droplets are considered to be of spherical shape, are not allowed to coalesce, deform, splash or break up. Ghenai and Lin [28] prescribed the number of particle trajectories, density, size and distribution of water droplets. Hasanzadeh et al. [35] solved the equation of motion of the (spherical) droplets using a Runge-Kutta type scheme after estimating their drag from the previously computed flow field solution. Iuliano et al. [45] followed a similar approach and neglected the interaction of the particle liquid phase on the gas phase (one way interaction). Honsek et al. [38] accounted for droplet deformation and breakup using the semi-empirical correlations of Clift et al. [15] and Pilch [72] respectively. Jung and Myong [47] proposed a second order finite volume upwind scheme that facilitates the computation of the problem using an Eulerian formulation only. Rendall and Allen [80] used a Lagrangian formulation for the droplets but developed a finite volume representation for the streamlines that speeded up the global algorithm convergence procedure.

Many other studies have been conducted on phenomenon related to SLD icing. Measurements of the ice roughness in short duration SLD events can be found in [61] and for example Andersen and Feo started to investigate the water film thickness for SLD conditions [6], since the thickness of the water film formed over the surface due to impingement of the supercooled water is an important parameter in aircraft icing modeling. He covers only a narrow range of conditions compared to the real SLD icing problem but in a later study [5] a wide range of experiments for different icing 
conditions were conducted at Cranfield University. They focus on the SLD conditions where the droplets are larger and other effects like splashing and re-impingement could occur. They study the thickness and the stability of the water film and they observed that in the SLD conditions, a continuous water film is not always formed over the surfaces and depending on the conditions a continues water film, rivulets or only individual beads over the target may be seen. They thought that the following parameters were the most important in the wetting form over the surfaces:

- The nature of the surface, surface energy and the hydrophobicity of the surface

- The size of the incoming droplets

- Liquid water content

- Impact velocity (normal component)

Again, the size of the incoming droplets appears to be an important parameter. If breakup prior to impingement took place, the droplet size of the incoming droplets would change drastically and the impact velocity and the impact location would also be modified. Aircraft manufactures have already reported in-flight observations of water droplet breakup near the wing surfaces of large transport aircraft [85], so a better understanding of these phenomena is required to improve analytical models and numerical simulation tools for SLD [63].

In the last SAE conference in 2015 on icing, several papers were dedicated to SLD problem, such as Alekseyenko et al. [2], Shimura and Yamamoto [83] and Chang et al [13]. The first one [2] was dedicated to study the interaction of SLD with an icing airfoil surface while in the second [83] the effect of droplet deformation models on SLD icing was investigated by means of simulation. The model of the drag coefficient was confirmed to influence the ice shape, the droplet impinging limit and the icing limit. Chang et al [13] discussed the breakup modes of SLD and the typical icing condition. They found that the main breakup modes involved are bag-breakup, multimode breakup and shear breakup, and they proposed a combined breakup model for the three models where the transition for each breakup mode was determined by the Weber number. For the bag, multimode and shear breakup model, the BTB model [96], the MMB model [95] and the DDB model [43] were considered. They concluded that much work is still required to improve and develop models for droplet breakup.

In this context, Vargas and Feo [93] have recently published an experimental study in which a rotating arm facility with an airfoil placed at the arm length and equipped with a high-speed imaging system was used to gather information on a series of water droplet global parameters as they intersected the airfoil path. Though water droplets were at ambient temperature, this experiment, that involved extensive visualization, could be considered as a first approach providing experimental insight into the phenomenon. However, it only covers droplets diameters up to 500 microns and one airfoil chord. Further studies are needed in this field including different airfoils sizes and droplet sizes up to $3 \mathrm{~mm}$ of diameter in order to study the size range of SLD encountered [93].

SLD ice accretion is a problem in aviation since the fatal accident in 1972. New phenomenon neglected for smaller droplets need now to be studied. These phenomenon include droplet breakup due to the presence of the flow field generated by the incoming airfoil. Droplet breakup changes drastically the droplet sizes which account for the ice accretion. Though, only water droplet at air temperature have been investigated, the 
present thesis aim to provide information that could benefit the research community that develops software for in-flight icing applications on this phenomena.

\subsection{OBJECTIVES OF THE THESIS}

The general objectives of the thesis are:

1. To understand the process of droplet deformation and eventual breakup when the droplet is exposed to a continuously accelerating flow field.

2. To lay a methodological basis to study this kind of problem, including experimental tests, in which further studies can be laid to obtain future engineering models.

The specific objectives are:

1. To provide experimental observation visualization on deformation and breakup of droplets of diameters from 300 to $3600 \mu \mathrm{m}$ in the stagnation line of an incoming airfoil. Three different model airfoils and five airfoil velocities were used.

2. To characterize the velocity and acceleration flow fields in the vicinity of the leading edge of the airfoil using Particle Image Velocimeter (PIV) so as to obtain the principal flow parameters involved in the problem of deformation and breakup of droplets in this flow field.

3. To collapse the experimental data and obtain the dimensionless parameters that govern the deformation and breakup process so as to provide insight into the underlying physics.

4. To generalize and densify reliable and clean databases on droplet deformation and breakup, using High Order Singular Value Decomposition as a data reduction method, which will be the starting point for future investigations.

5. To propose a method to determine the occurrence of the breakup of droplets in the vicinity of a leading edge of an airfoil given the characteristic of the flow field and the droplet.

6. To characterize the regimes of breakup likely to occur and the conditions that determine the limits for each regime in the special situation of an accelerating flow field previously studied. 


\section{Chapter 2}

\section{DESCRIPTION OF THE EXPERIMENTAL SETUP}

\subsection{EXPERIMENTAL SETUP OF THE DROPLET DE- FORMATION AND BREAKUP TESTS}

Three experimental campaigns were carried out to obtain the droplet deformation and breakup evolution in a flow field generated by an incoming airfoil at INTA rotating arm facility in Spain near Madrid. The conceptual approach of all experiments campaigns were the same, see figure 2.1. Droplets of the same size were generated by a droplet generator and fall vertically as a stream where droplets are equal spaced. At the same time, an airfoil model is moving towards the droplet at constant velocity $U_{m}$. The air in front of the model, where droplets are, is moving because of the movement of the airfoil, at a velocity $U_{\text {air }}$ whose magnitude depends on the distance to the model, which in turn depends on time. The actual phenomenon studied is the one encountered along the stagnation line of the airfoil. The sketch of the experimental setup is shown in figure 2.2.

In the first two campaigns, a high speed camera recorded the phenomena using the shadowgraph technique, while in the third experimental campaign a high resolution camera using direct illumination was utilized. Regarding the droplet size, only droplets up to $750 \mu \mathrm{m}$ of radius were investigated during the first experimental campaign, while

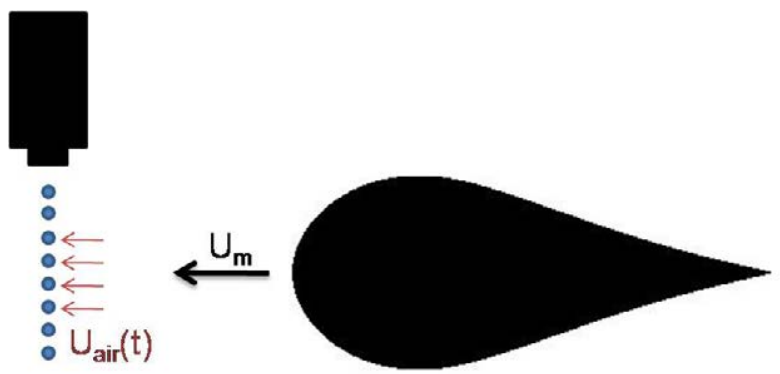

Figure 2.1: Conceptual view of the experiments 


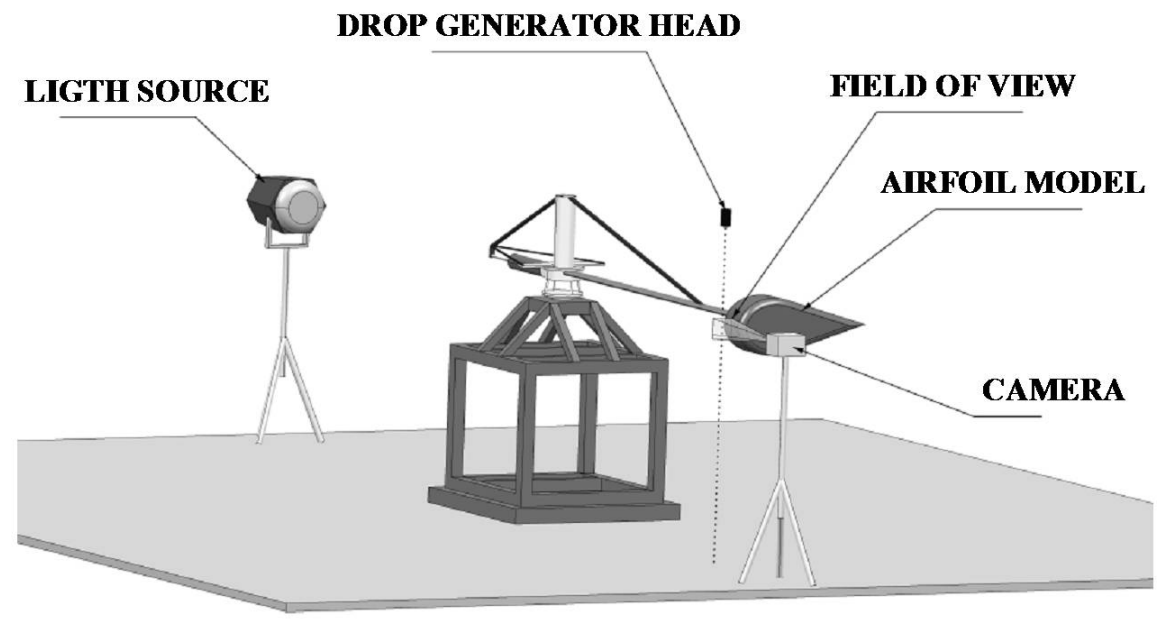

Figure 2.2: Sketch of experimental setup of the experiments

in the second and third campaigns the droplet radius studied increased up to $1500 \mu \mathrm{m}$.

In the following sections, each of the components of the experimental setup, namely, rotating arm facility, airfoils models, droplet generator and the data acquisition system, will be described in detail. Then, the experimental setup for the measurements of the flow field that the droplets actually see will be described. And finally, the process to analyze and show the data obtained during the shadowgraph experiments, which was conducted mainly by the development of an in-house software named SITEA, will also be described.

\subsubsection{Rotating Arm Facility}

This rotating arm unit is made up of an electric motor, a support structure and a rotating arm, see figure 2.3. The electric motor has a power of $5 \mathrm{~kW}$ and it is placed inside the support structure. The motor axle is vertical. The support structure is connected to the floor via four slip ring vibration dampers that contribute to stabilize the overall system. The rotating arm length measured from the rotation center to the center of the leading edge of the model is $2.2 \mathrm{~m}$. A strut system is mounted opposite to the arm for balance and vibration control purposes. A LED optical system mounted on the support structure points a light beam towards the arm path in such a way that its reflection is collected by a detector, thereby allowing for the monitorization of the rotational velocity of the arm. The maximum rotational velocity that can be achieved is $400 \mathrm{rpm}$, which means that the maximum translational velocity at the rotating arm end is $90 \mathrm{~m} / \mathrm{s}$ (Mach 0.26). The rotational velocity can be changed at discrete steps in such a way that the translational velocity can be varied at intervals of $1 \mathrm{~m} / \mathrm{s}$. An additional optical trigger is present that sends a signal when the model passes at a certain location. This trigger is important to synchronize the airfoil model and the data acquisition system. 


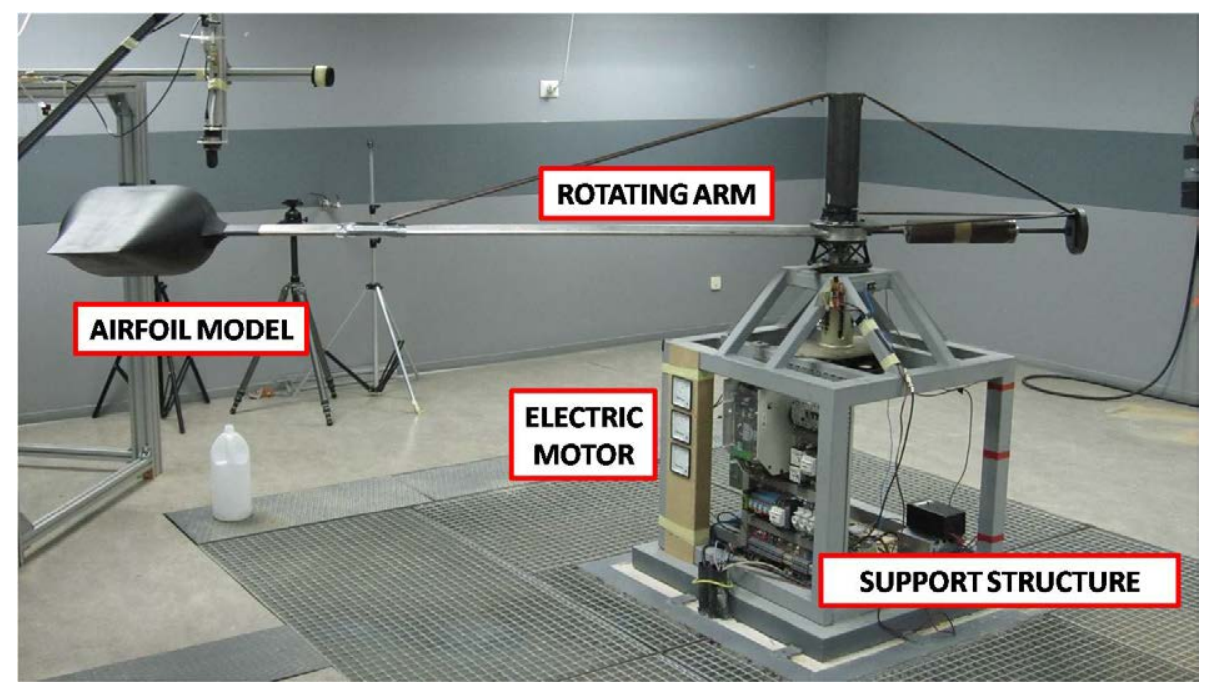

Figure 2.3: Photograph of the experimental setup in the rotating arm facility

\begin{tabular}{cccc}
\hline Model & Chord $(\mathrm{c})$ & Leading edge radius $(\mathrm{Rc})$ & Thickness $(\mathrm{Tc})$ \\
\hline M1 & $0.690 \mathrm{~m}$ & $0.103 \mathrm{~m}$ & $0.276 \mathrm{~m}$ \\
M2 & $0.468 \mathrm{~m}$ & $0.070 \mathrm{~m}$ & $0.187 \mathrm{~m}$ \\
M3 & $0.199 \mathrm{~m}$ & $0.030 \mathrm{~m}$ & $0.080 \mathrm{~m}$ \\
\hline
\end{tabular}

Table 2.1: Geometry parameters of the three airfoils: M1, M2, and M3

\subsubsection{Airfoils Models}

Three airfoils models of the same geometry but different size, labeled as M1, M2 and M3, were attached to the end of the rotating arm during the tests. The airfoil profile used for them was the TURN T/D 0.4 found in [4], which is rather thick and blunt so as to reproduce the actual scale of the problem more accurately. The shape parameters for each airfoil model and the dimensionless airfoil's coordinates are specified in tables 2.1 and 2.2 respectively. The span for all the three models was $200 \mathrm{~mm}$ and at each side, as observed in figure 2.4 , the models were finished with a revolution surface from the airfoil profile. Some views of the geometry of one airfoil are presented in figures 2.4 and 2.5. The models were made up of Styrofoam by a hot wire system, except for a metal plate located in the leading edge to reinforce the surface where droplets impact.

\subsubsection{Droplet Generator}

Two droplet generators were used during the experiments. For the first experimental campaign, only a monosized TSI MDG-100 droplet generator was used. However, for the second and third experimental campaign another in-house generator was also used in order to generate bigger droplets. Both devices were based on the Rayleigh theory [79]. Due to axisymmetric disturbances a laminar jet can break up into droplets. Using a linearized stability analysis Rayleigh showed that for wavelengths smaller than the circumference of the jet no break-up occurs, however for greater wavelengths the disturbance grows exponentially and causes the jet to break up. He also gave the optimal wavelength with the fastest growing disturbance for inviscid fluid being $d_{j}$ the jet 


\begin{tabular}{cccc}
\hline $\mathrm{x} / \mathrm{c}$ & $\mathrm{z} / \mathrm{c}$ & $\mathrm{x} / \mathrm{c}$ & $\mathrm{z} / \mathrm{c}$ \\
\hline 1.000 & 0.000 & 0.241 & 0.200 \\
0.937 & 0.014 & 0.211 & 0.196 \\
0.877 & 0.028 & 0.183 & 0.191 \\
0.818 & 0.044 & 0.157 & 0.183 \\
0.762 & 0.059 & 0.133 & 0.173 \\
0.707 & 0.076 & 0.110 & 0.162 \\
0.655 & 0.093 & 0.090 & 0.150 \\
0.604 & 0.110 & 0.072 & 0.136 \\
0.556 & 0.127 & 0.056 & 0.121 \\
0.510 & 0.143 & 0.042 & 0.105 \\
0.465 & 0.158 & 0.030 & 0.089 \\
0.423 & 0.171 & 0.020 & 0.072 \\
0.383 & 0.183 & 0.012 & 0.055 \\
0.344 & 0.192 & 0.006 & 0.041 \\
0.308 & 0.198 & 0.002 & 0.032 \\
0.274 & 0.200 & 0.000 & 0.000
\end{tabular}

Table 2.2: Dimensionless coordinates of the airfoils.
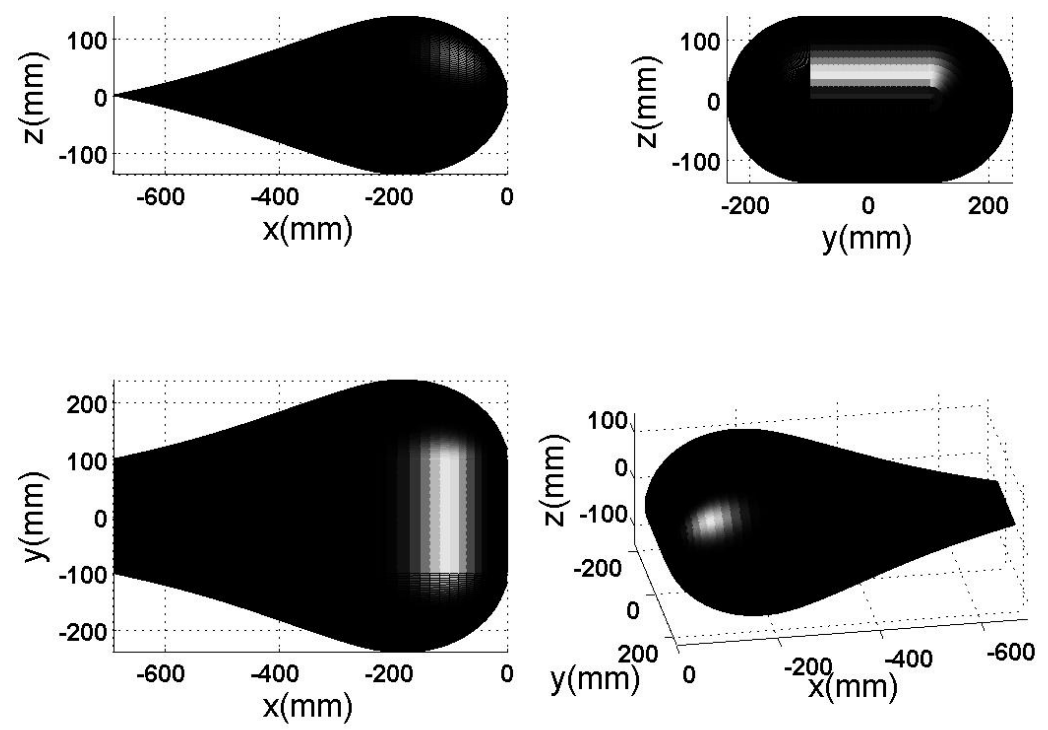

Figure 2.4: Side (top left), front (top right), top (bottom left) and perspective (bottom right) views of the airfoil model M1. 


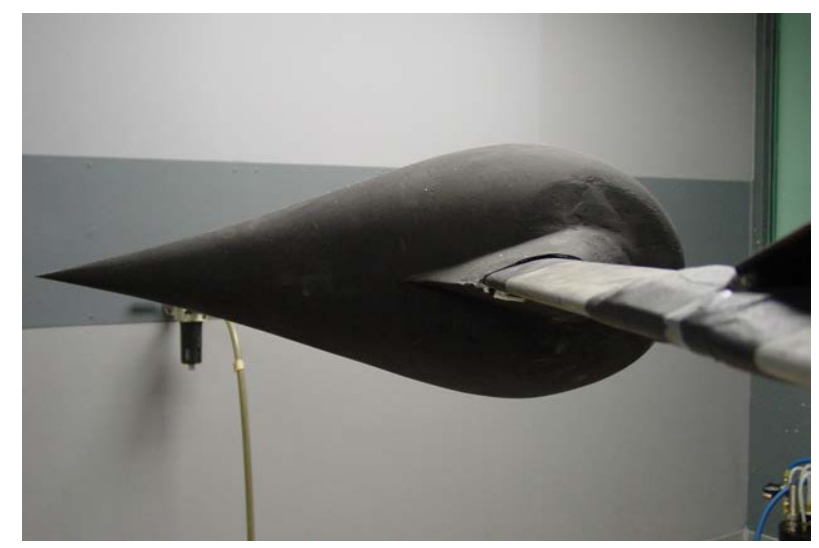

Figure 2.5: Airfoil model photograph.

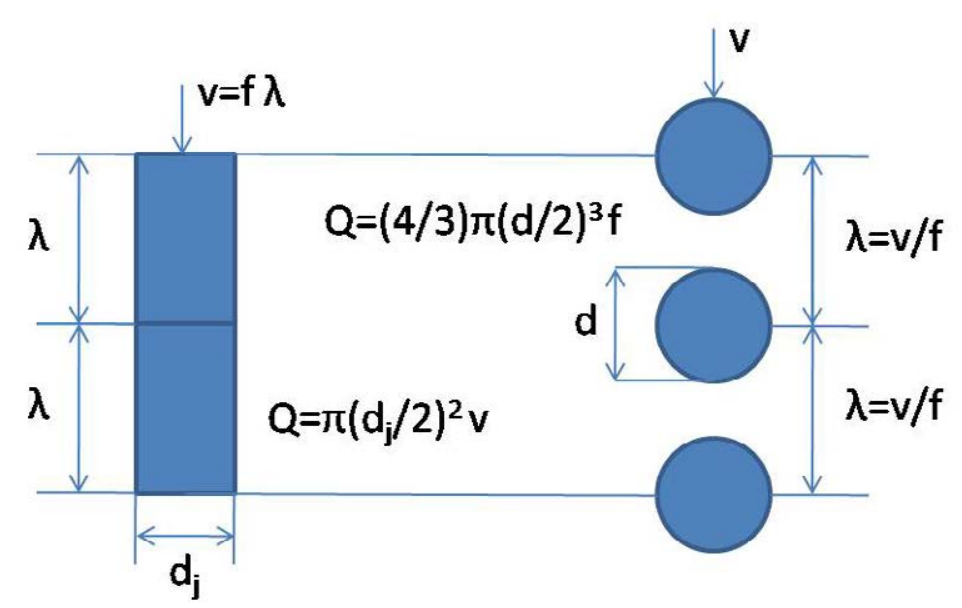

Figure 2.6: Monodisperse Droplet Diameter Calculation

diameter.

$$
\lambda_{o p t}=\pi \sqrt{2} d_{j} \cong 4,44 d_{j}
$$

If a sinusoidal disturbance of the frequency corresponding to the the optimal wavelength $\lambda_{o p t}$, the so-called Rayleigh frequency, is applied to the jet, then, a monosized stream of droplets is generated at this frequency rate. By conservation of the mass flow, see figure 2.6 it is deduced that the liquid cylinder of one wavelength forms one droplet, which allows for the calculation of the droplet diameter of the monodisperse stream $d$ as a function of the jet flow $Q$ and the vibrating frequency $f$.

$$
d=\sqrt[3]{\frac{6 Q}{\pi f}}
$$

Additionally, another relation is obtained from the mass flow conservation that relates the droplet and the jet diameters: 


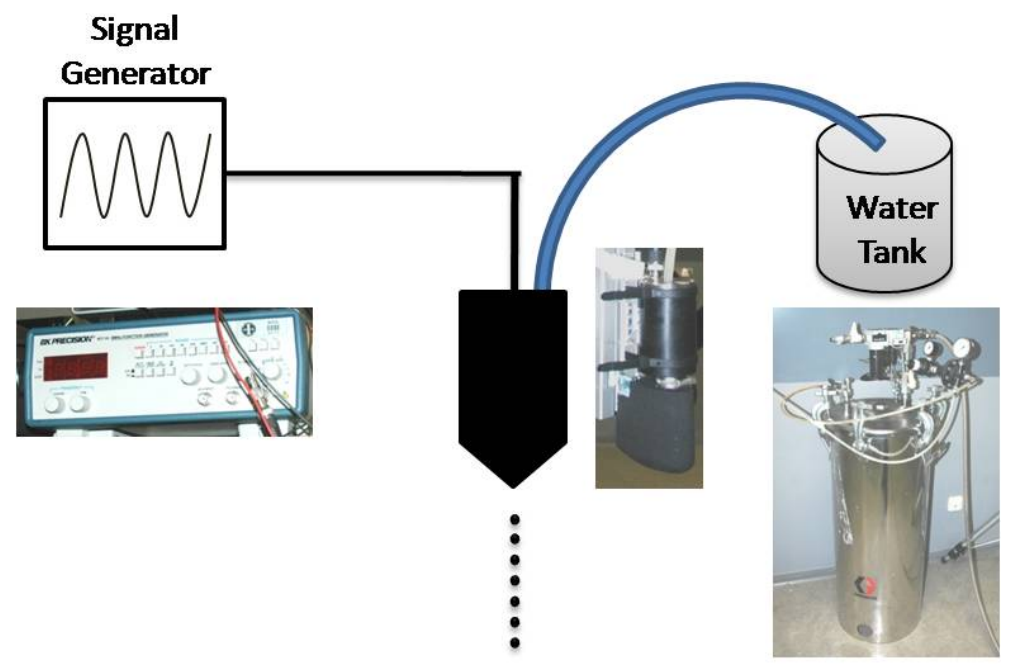

Figure 2.7: TSI Monodisperse Scheme

$$
d=\sqrt[3]{1,5 \lambda d_{j}^{2}}
$$

For the Rayleigh frequency $\left(\lambda=\lambda_{\text {opt }}=4,44 d_{j}\right)$, the droplet diameter is 1.88 times the jet dimater. A range of frequencies near the Rayleigh frequency and jet velocities exists where uniform sized droplets can be formed [62]. This means that for a desired droplet diameter, only a limited range of output orifice diameter can be used. The droplet radius was chosen in the range from $200 \mu \mathrm{m}$ to $1500 \mu \mathrm{m}$ by modifying the jet mass flow rate, the output orifice diameter and the vibration frequency. In the monosized TSI MDG-100 a piezoelectric is used to vibrate the jet at a given frequency. The output of a function generator, where frequency is selected, is connected to the droplet monodisperse. A calibrated orifice in the output is used to generate the desired jet diameter. In the first experimental campaign, the water came from a water tank by a pump, which was regulated to obtain the desired jet flow for a given orifice diameter. In the other experimental campaigns, the water came from a pressurized water deposit, where the output pressure was chosen. By modifying the input flow and the output orifice, the flow jet was selected. An scheme of this monodisperse in the second campaign is shown in figure 2.7 .

Another mononodisperse was used in order to obtain bigger droplets. The scheme of this in-house developed monodisperse is shown in figure 2.8. It can be observed in this figure, that the signal needs now to be amplified to be used to move an actuator that actually vibrates at the desired frequency and that is connected to the jet flow. A pump is used to provide the water flow into the monodisperse generator and a pressure gauge indicates the input pressures on the droplet generator head.

Prior to the experiments, both systems were calibrated by measuring the flow jet for each calibrated output orifice and either the pump voltage, or the output deposit pressure depending on the water feeding system (see figure 2.9). Then, the frequency for each desired droplet diameter was calculated and different amplitudes of the wave connected to the vibrating system were tested to obtain the best configuration. Finally the actual droplet diameters for each configuration were measured by recording ima- 


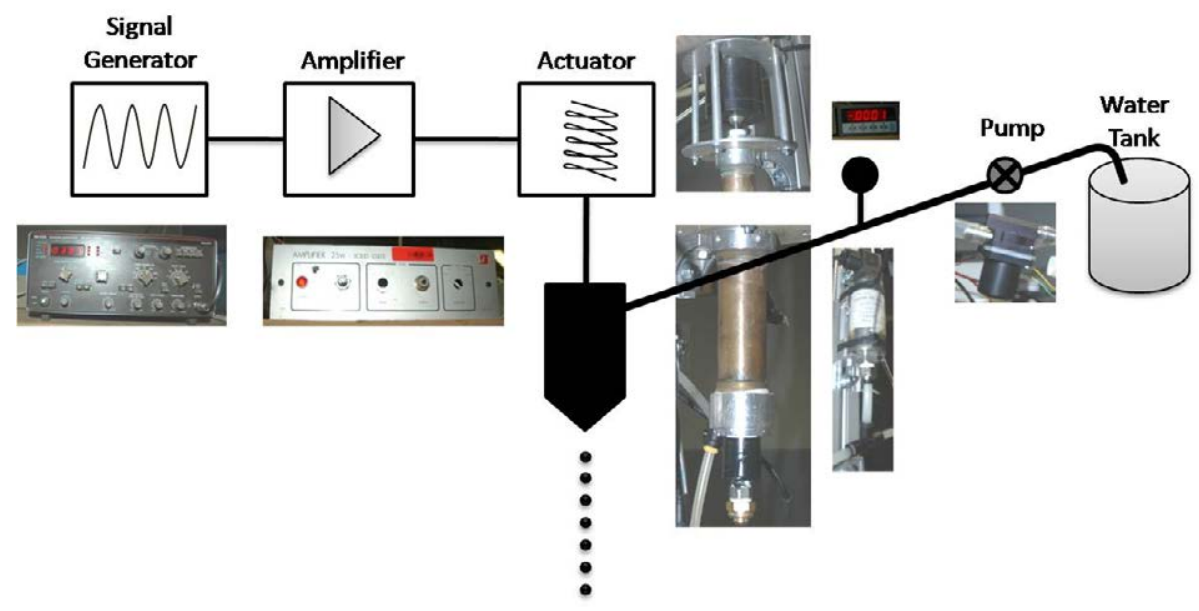

Figure 2.8: INTA Monodisperse Scheme

ges. Two examples of the images recorded for the calibration, one per each droplet generator, are shown in figure 2.10 .

\subsubsection{Data Acquisition System}

Two different data acquisition systems were used during the experiments. During the first two campaigns a high speed camera was used to record the phenomenon using shadowgraph technique, while in the third campaign a high resolution camera and and direct flash illumination were used.

\section{Data Acquisition System 1}

The data was acquired by means of recorded images by a high speed video camera. The data acquisition system consisted of a high speed video camera and a powerful light source aligned such that the droplets were illuminated from an appropriate direction to generate a shadowgraph in a white-gray background. This is know as shadowgraph illumination technique. A photograph of this Data acquisition system 1 is shown in figure 2.11

The camera used was a Photron $S A-5$. Its image capturing rate could be selected in the range from 1000 frames per second (fps) to 1,000,000 fps. The pixels resolution decreases as the number of captured frames per second increases. In the actual experiments a compromise had to be reached between the two control variables. In particular, the image capturing rate was 75,000 fps during the first experimental campaign an $72000 \mathrm{fps}$ during the second experimental campaign which implied a resolution of 192 x 312 pixels and 256 x 512 pixels respectively. The maximum shutter speed was 1 $\mu s$. The software used for image capturing was Photron FASTCAM. Extra lenses were used for additional magnification of the images. This configuration consisted of a $200 \mathrm{~mm}$ Micro Nikkor lens with a 2x doubler located in between the camera and the $200 \mathrm{~mm}$ lens. The magnification was obtained by placing the camera at a distance of $1.47 \mathrm{~m}$ away from the droplets paths. The light source was a $2000 \mathrm{~W}$ xenon light. An example of a sequence of the recorded images can be seen in figure 2.12. 

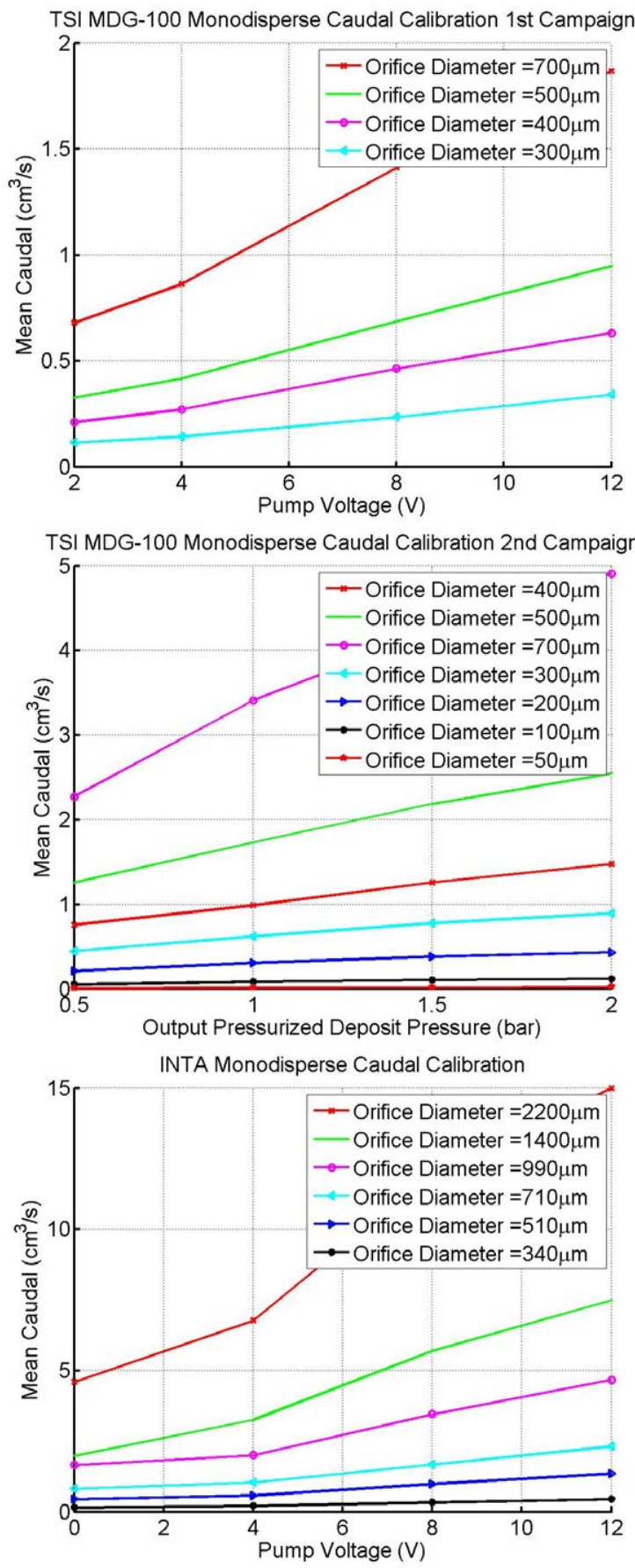

Figure 2.9: Above: Caudal measurements for the TSI MDG-100 Droplet Generator for each pump voltage (1st experimental campaign) and output orifice diameter. In the middle: Caudal measurements for the TSI MDG-100 Droplet Generator for each output pressurized deposit pressure(2nd experimental campaign) and output orifice diameter. Below: Caudal measurements for the In-House Droplet Generator for each pump voltage and output orifice diameter. 

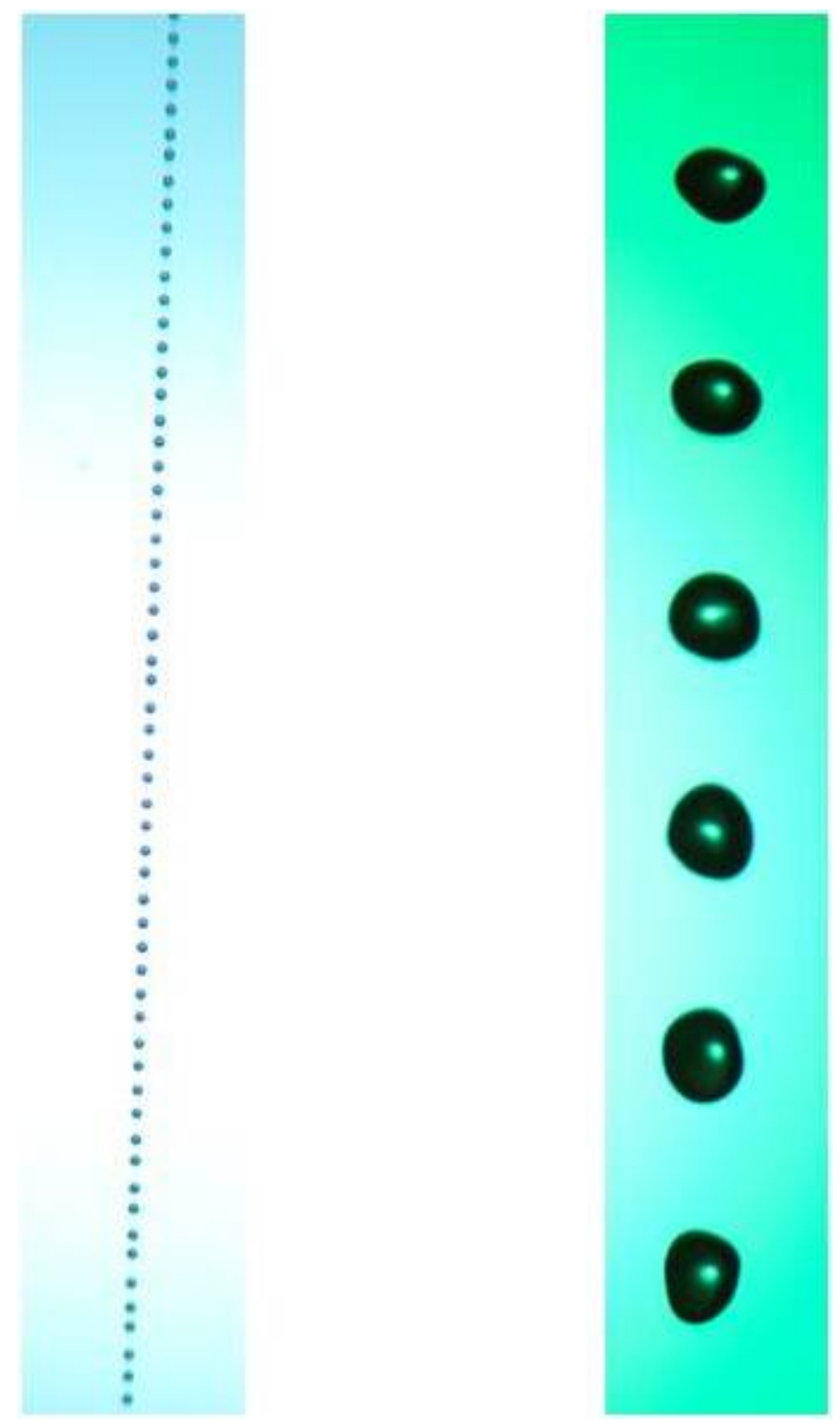

Figure 2.10: Examples of the images recorded for the calibration of the droplet generator. On the left: Droplet Diameter: $450 \mu \mathrm{m}$, TSI MDG-100 generator, Orifice Diameter $=300 \mu \mathrm{m}$, Wave Frequency $=12.76 \mathrm{KHz}$, Wave Amplitude $=20 \mathrm{~V}$, Deposit Output Pressure $=0.8$ bares. On the right: Droplet Diameter: $2800 \mu \mathrm{m}$, In-house droplet generator, Orifice Diameter $=1.7 \mathrm{~mm}$, Pump Voltage $=8 \mathrm{~V}$, Caudal $=8,33 \mathrm{~cm}^{3} / \mathrm{s}$, Wave Frequency $=0,72 \mathrm{KHz}$, Wave Amplitude: $12 \mathrm{~A}$ 


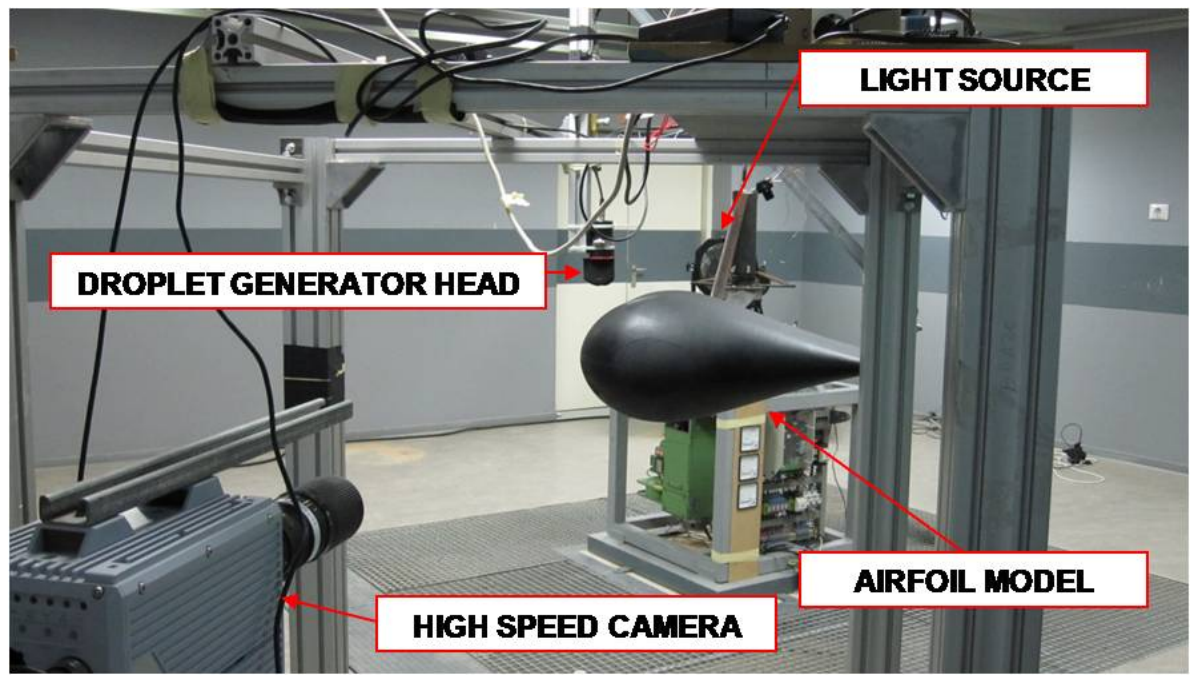

Figure 2.11: Data Acquisition System 1

\section{Data Acquisition System 2}

The aim of this acquisition system is to gain resolution in the images of droplet breakup in order to study the morphology of the breakup. For this reason this acquisition system consisted of a high resolution camera, a flash illumination source, a trigger and a hub. An optical trigger is situated during the path of the model such that a trigger signal is sent when the model passes by a certain position. In figure 2.13 a photograph of this acquisition system is shown. Two stroboscopic microflashes were used to illuminate the droplets from above and below at the same time. The camera used was the Hasselblad H3DII-39.An example of a sequence of the recorded images can be seen in figures 2.14 and 2.15 .

\subsection{EXPERIMENTAL SETUP OF THE BASE FLOW MEASUREMENTS}

Prior to the deformation and breakup tests described in the previous chapter, in order to characterize the flow field that the droplet actually see, the flow field velocity generated by the airfoil and without the presence of the droplets was measured in advance by means of Particle Image Velocimetry (PIV). In the following sections the PIV system, the experimental setup for these measurements and the uncertainty will be described.

\subsubsection{PIV System}

The PIV system is a non-intrusive velocity measurement technique based on the idea of taking two images in two consecutive known instants of a seeded flow by illuminating with two laser pulses $[1,75]$. Each laser pulse is recorded in a different image, and these two images are cross-correlated to obtain the displacement of the particles (used to seed the flow) in each sub-interrogation area (interrogation spot). The theory 


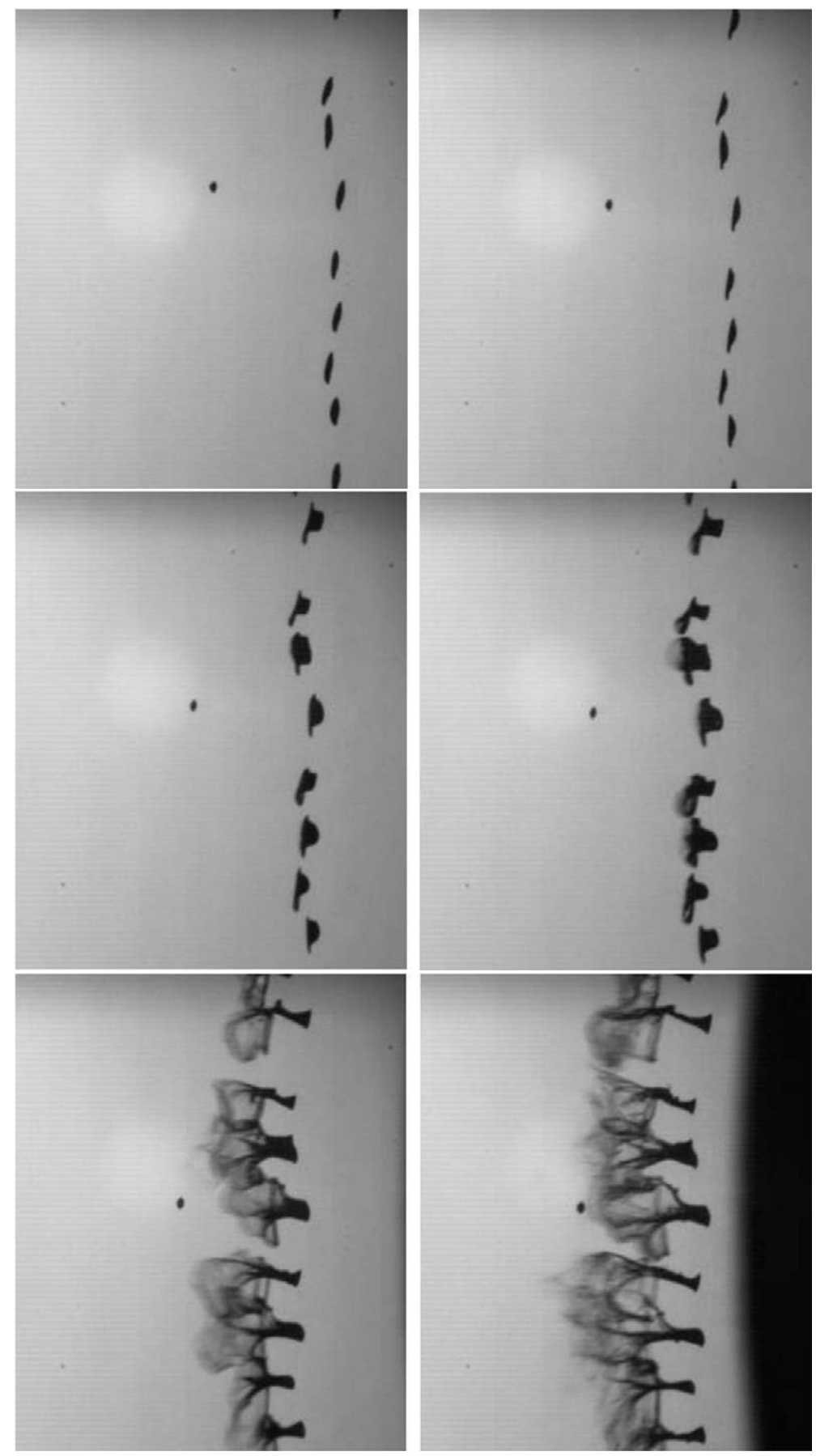

Figure 2.12: Example of the evolution of the recorded images along time for a droplet of $400 \mu \mathrm{m}$ of radius, where the $\mathrm{M} 1 \mathrm{model}$ was moving at $80 \mathrm{~m} / \mathrm{s}$. 


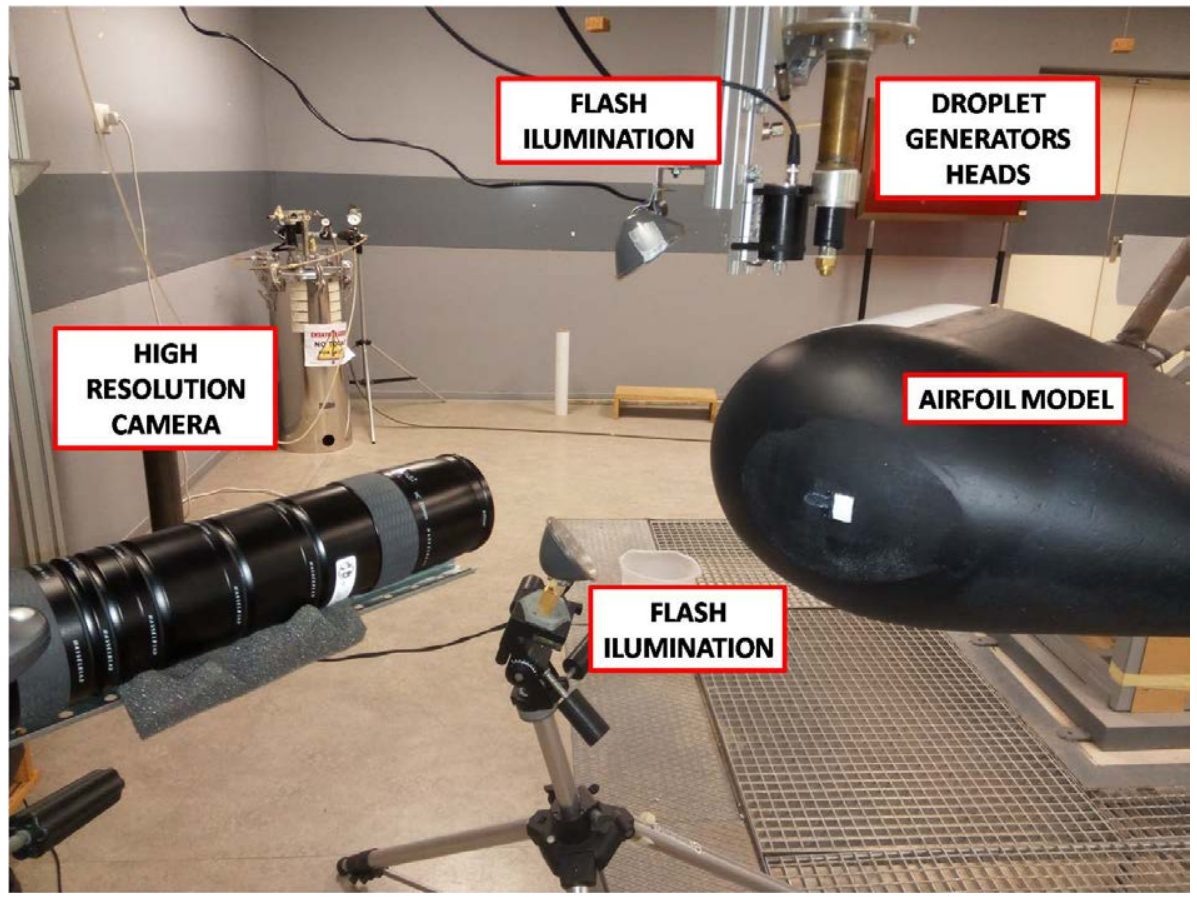

Figure 2.13: Data Acquisition System 2.

of the cross-correlation can be found in [48]. Knowing the time between the two consecutive laser pulses, the velocity for each sub-interrogation area is calculated assuming constant velocity during the time between pulses. The time between pulses was chosen for each experiment so that the particle displacement was less than a quoter of the size of the sub-interrogation area, according to Prasad [74]. An sketch of the apparatus involved in a PIV system is shown in figure 2.16.

The PIV set used was a TSI system. Flow illumination was provided by two pulsed Nd:YAG $190 \mathrm{~mJ}$ laser. Each laser pulse lasted for $8 \mathrm{~ns}$. Images were taken using a Power View Plus 4MP camera with a resolution of 2048 x 2048 pixels. Data transmission was made directly to the PC RAM via a 64-bit camera link which allows for an information transfer up to $512 \mathrm{MB}$ per second, which is sufficient for the information budget considered. The camera lens were AF-S VR Micro-Nikkor $105 \mathrm{~mm} \mathrm{f} / 2.8$ G IF-ED Nano Crystal Coat, AF Nikkor $80.200 \mathrm{~mm}$ f/2.8 D IF-ED and Nikkor $50 \mathrm{~mm}$ $\mathrm{f} / 1.4$. The flow in the test room was seeded with olive oil droplets having a diameter of $1 \mu \mathrm{m}$, which is small enough to follow the flow. Synchronization between image capturing and flow illumination and the analysis was carried out using the TSI Insight $3 \mathrm{G}$ software and a Laser Pulse synchronizer.

\subsubsection{PIV Experimental Setup}

The vertical plane (VP) tangent to the airfoil trajectory that intersects the droplet generator and the horizontal plane (HP) intersecting the airfoil leading edge were illuminated, see figures 2.17 and 2.18. At that very moment, camera lenses having different focal distances (FD) recorded fields of vision (FOV) of different sizes, the interrogation windows, in the respective illuminated planes (either the vertical or the horizontal 


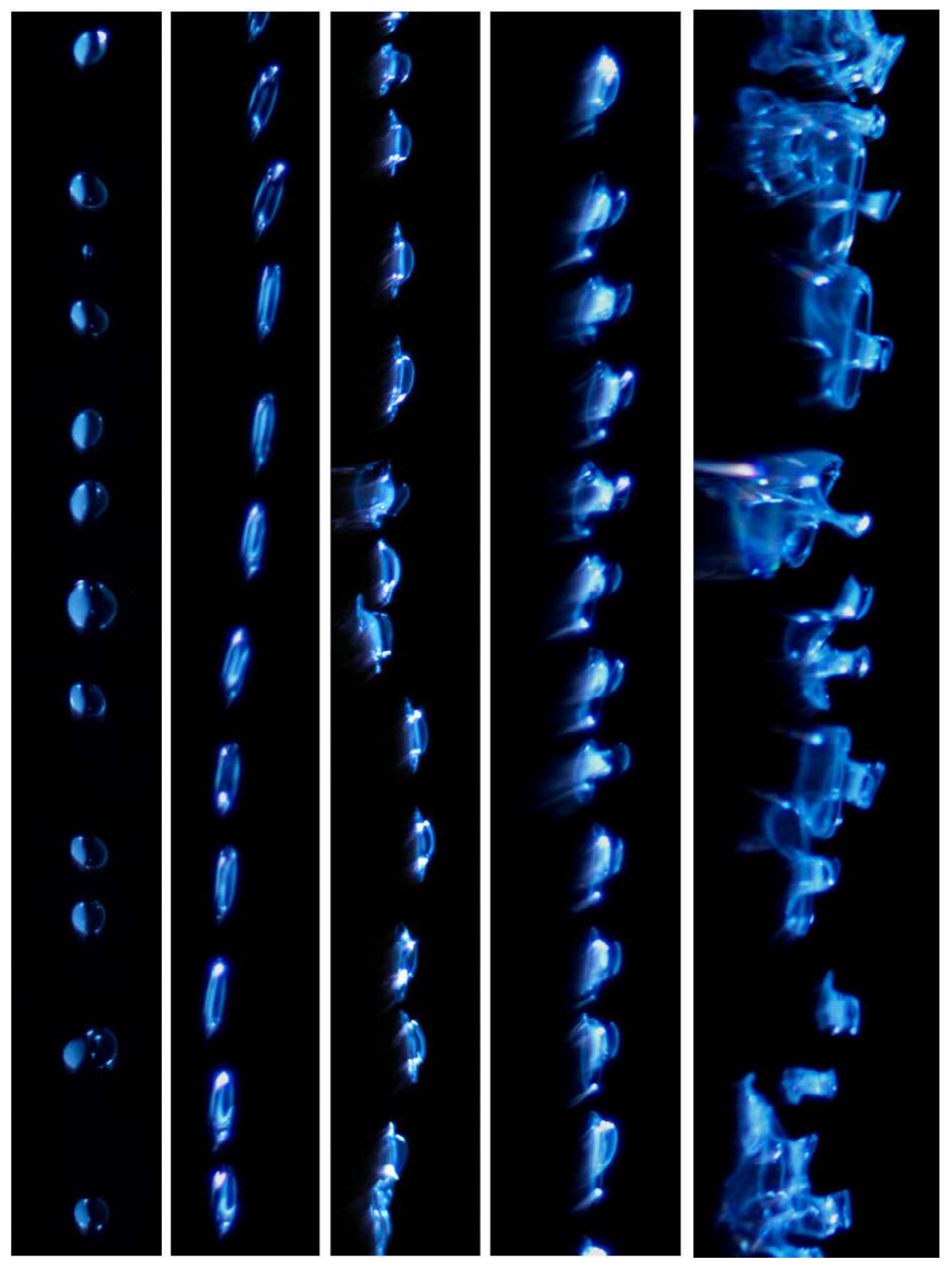

Figure 2.14: Example of images obtained using the Data Acquisition 2. 

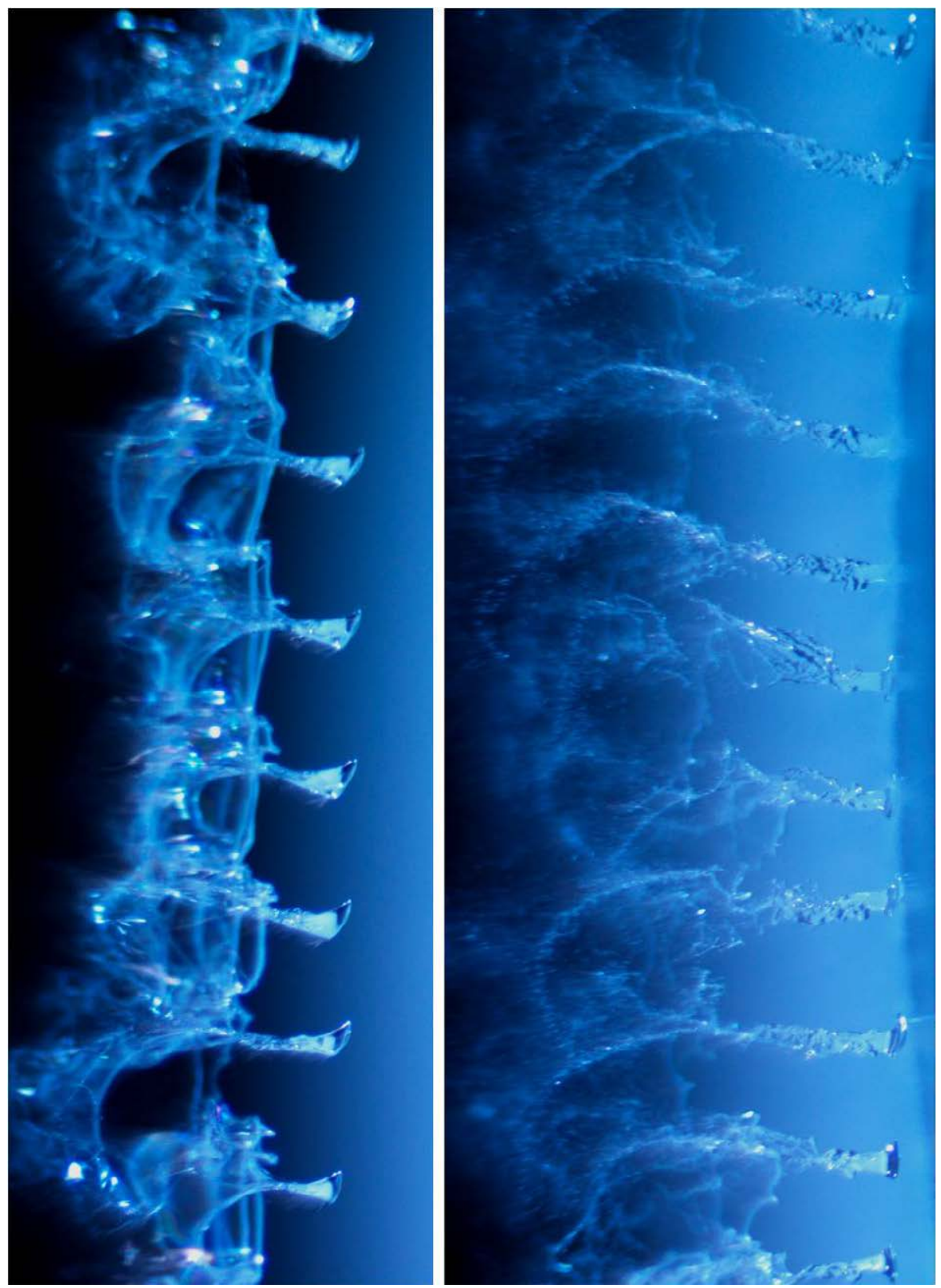

Figure 2.15: Example of images obtained using the Data Acquisition 2. 


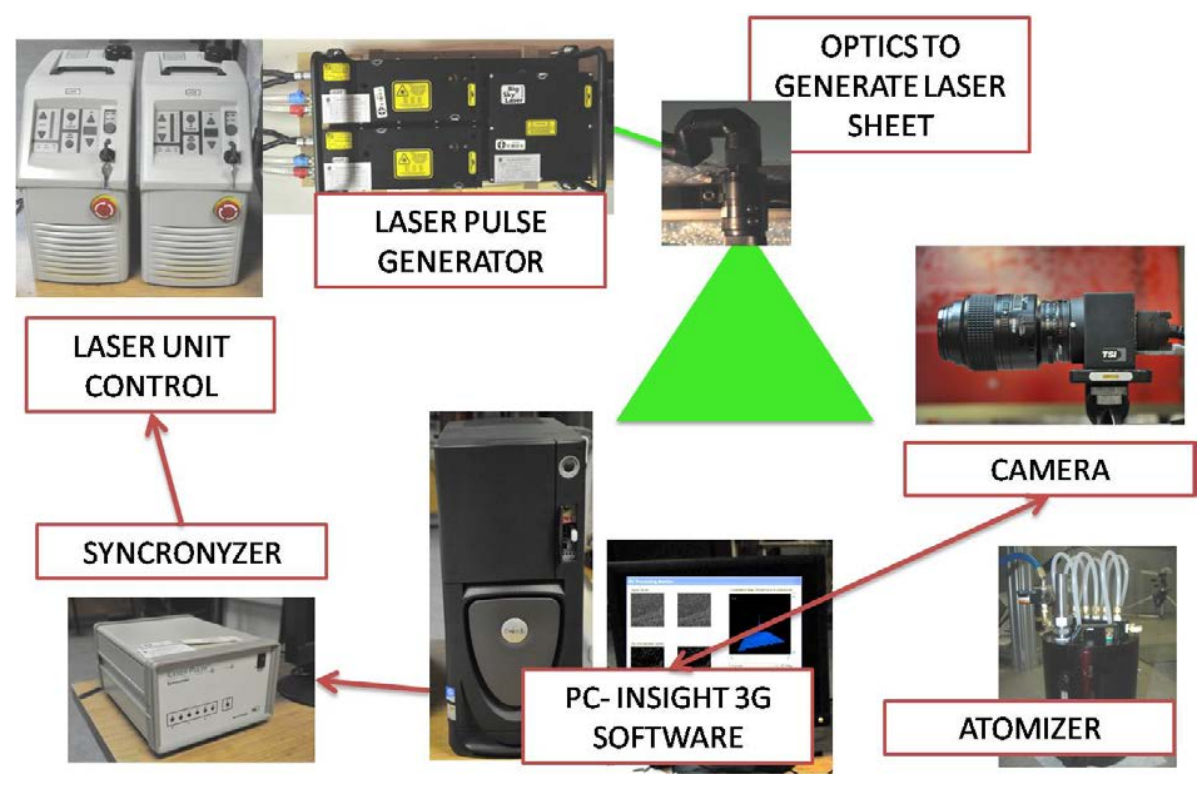

Figure 2.16: Sketch showing the PIV system

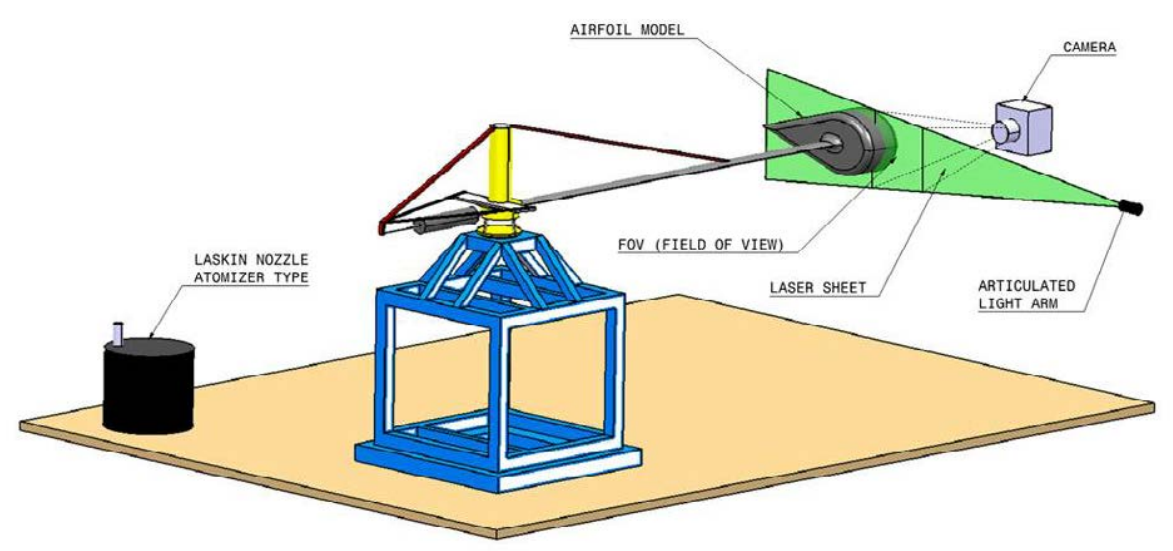

Figure 2.17: Sketch showing the experimental PIV setup for the vertical plane interrogation window in the rotating arm facility 


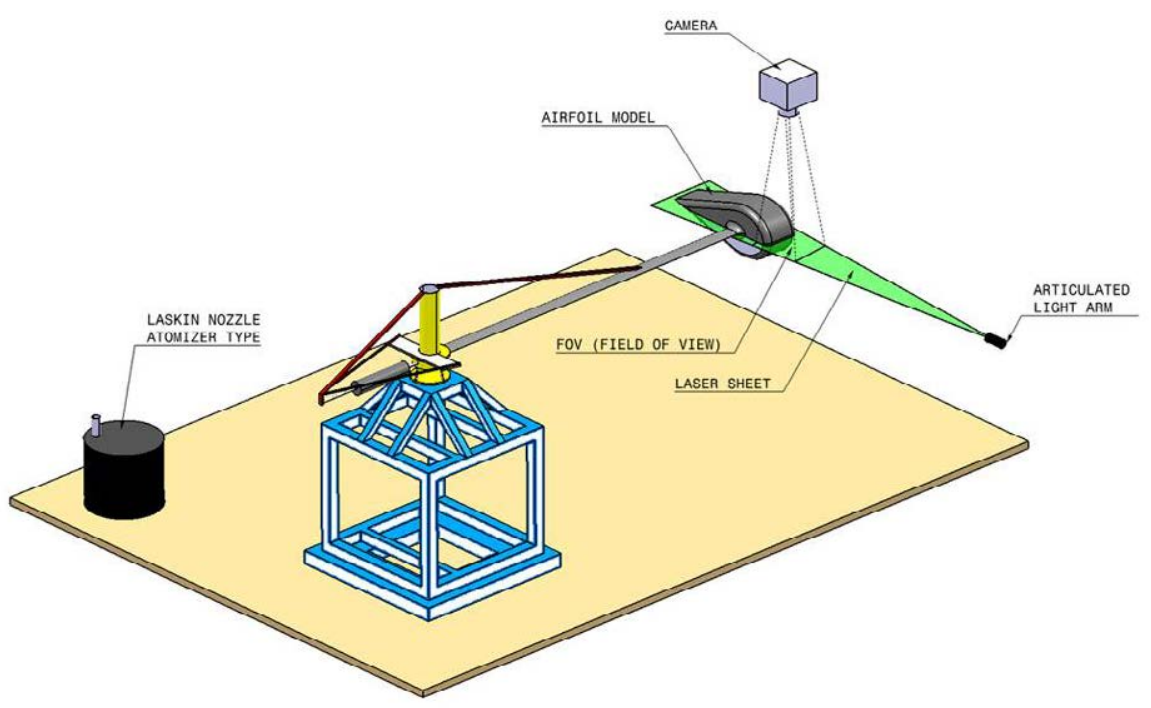

Figure 2.18: Sketch showing the experimental PIV setup for the horizontal plane interrogation window in the rotating arm facility

plane). Relations between FD and FOV were as follows:

- $\mathrm{FOV}=30 \mathrm{~mm} \times 30 \mathrm{~mm}, \mathrm{FD}=200 \mathrm{~mm}, \mathrm{VP}$.

- $\mathrm{FOV}=60 \mathrm{~mm} \times 60 \mathrm{~mm}, \mathrm{FD}=105 \mathrm{~mm}, \mathrm{VP}$.

- $\mathrm{FOV}=200 \mathrm{~mm} \times 200 \mathrm{~mm}, \mathrm{FD}=105 \mathrm{~mm}$, VP.

- $\mathrm{FOV}=400 \mathrm{~mm} \times 00 \mathrm{~mm}, \mathrm{FD}=50 \mathrm{~mm}, \mathrm{VP}$.

- $\mathrm{FOV}=200 \mathrm{~mm} \times 200 \mathrm{~mm}, \mathrm{FD}=50 \mathrm{~mm}, \mathrm{HP}$.

The combination of a trigger and a synchronizer allowed for taking PIV images at the fixed interrogation windows for any position of the model airfoil during its circular trajectory, see figure 2.19 .

The summary of PIV test cases that were actually carried out is presented in table 2.3. The legend for the parameters shown in table 2.3 is as follows:

- $\lambda_{1}$ : model size and position of the interrogation window. M1 M2 and M3 correspond to the larger, intermediate and smaller airfoil model respectively. $\mathrm{V}$ and $\mathrm{H}$ refer to either vertical or horizontal position of the interrogation window (see figure 2.19).

- $\lambda_{2}$ : Size (in $\mathrm{mm}$ ) of the interrogation window (FOV).

- $\lambda_{3}$ : Velocity (in $\mathrm{m} / \mathrm{s}$ ) of the airfoil model.

- $\lambda_{4}$ : Pixel size $($ in $\mu m)$ in the interrogation window.

- $\lambda_{5}$ : Spot size (in mm).

- $\lambda_{6}$ : Number of samples taken. 

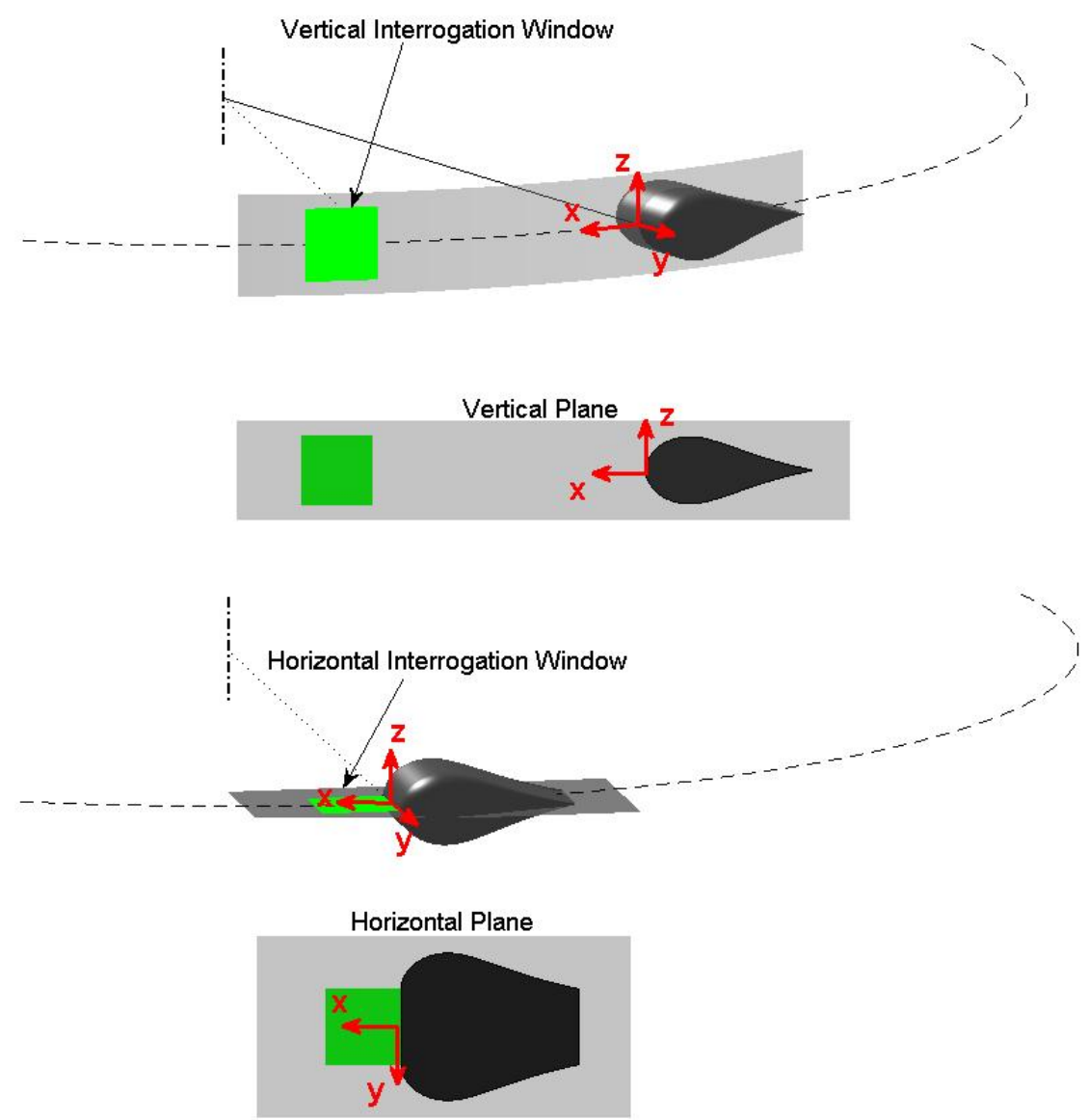

Figure 2.19: Sketch showing the interrogation windows and the coordinate system in the rotating arm facility 


\begin{tabular}{cccccc}
\hline$\lambda_{1}$ & $\lambda_{2}$ & $\lambda_{3}$ & $\lambda_{4}$ & $\lambda_{5}$ & $\lambda_{6}$ \\
\hline M1-V & $196 \times 196$ & 65 & 95 & 6.1 & 50 \\
M1-V & $196 \times 196$ & 89 & 95 & 6.1 & 50 \\
M1-V & $396 \times 396$ & 65 & 193 & 12.4 & 50 \\
M1-V & $396 \times 396$ & 89 & 193 & 12.4 & 50 \\
M1-V & $35 \times 35$ & 65 & 16 & 1.1 & 50 \\
M1-V & $35 \times 35$ & 89 & 16 & 1.1 & 50 \\
M1-H & $193 \times 193$ & 65 & 94 & 6.0 & 50 \\
M1-H & $193 \times 193$ & 89 & 94 & 6.0 & 50 \\
M2-V & $222 \times 222$ & 65 & 108 & 6.9 & 50 \\
M2-V & $222 \times 222$ & 92 & 108 & 6.9 & 50 \\
M2-V & $430 \times 430$ & 65 & 210 & 13.4 & 50 \\
M2-V & $430 \times 430$ & 92 & 210 & 13.4 & 50 \\
M2-V & $35 \times 35$ & 66 & 17 & 1.1 & 50 \\
M2-V & $35 \times 35$ & 92 & 17 & 1.1 & 50 \\
M2-H & $209 \times 209$ & 65 & 102 & 6.5 & 100 \\
M2-H & $209 \times 209$ & 92 & 102 & 6.5 & 100 \\
M3-V & $298 \times 208$ & 93 & 102 & 6.5 & 10 \\
M3-V & $62 \times 62$ & 92 & 30 & 1.9 & 10 \\
M3-V & $31 \times 31$ & 92 & 13 & 1.9 & 10 \\
M3-H & $210 \times 210$ & 92 & 103 & 6.6 & 10 \\
\hline
\end{tabular}

Table 2.3: Dimensionless coordinates of the airfoils.

Sampling of the flow field was carried out at frequencies in the range from $4.75 \mathrm{~Hz}$ to $6.5 \mathrm{~Hz}$ depending on the model velocities. The time $\Delta t$ between two consecutive laser pulses was varied in the range from $1.1 \mu \mathrm{s}$ to $200 \mu \mathrm{s}$. For example, in the case of the highest airfoil velocity $(90 \mathrm{~m} / \mathrm{s})$ and the larger $\Delta t$ for this velocity $(34 \mu \mathrm{s})$, a particle would move a distance of the order of $3 \mathrm{~mm}$ between consecutive laser pulses in the stream-wise direction that is much smaller than the characteristic length of the problem (the radii, $103 \mathrm{~mm}, 70 \mathrm{~mm}$ and $30 \mathrm{~mm}$, of the airfoil leading edge). Each PIV area was divided into smaller sub-interrogation areas containing 64pixels x 64pixels, which depending on the actual field of view correspond to geometrical areas having sizes of $1 \mathrm{~mm} \times 1 \mathrm{~mm}, 2 \mathrm{~mm}$ x $2 \mathrm{~mm}, 6 \mathrm{~mm}$ x $6 \mathrm{~mm}$ and $13 \mathrm{~mm}$ x $13 \mathrm{~mm}$ respectively. The spatial flow resolution of the experimental tests in the near region of the leading edge, at distances up to $30 \mathrm{~mm}$, was of the order of $1 \mathrm{~mm}$ that is considered to be sufficient to describe the phenomena being analyzed. At larger distances form the leading edge, the spatial flow resolution was of the order of $6 \mathrm{~mm}$ and $12 \mathrm{~mm}$ which is also considered to be sufficient to characterize the flow velocity field accurately enough. Each recording sequence consisted of 10/50/100 frames, one frame per revolution, separated from each other $0.21 \mathrm{~s}$ and $0.15 \mathrm{~s}$, corresponding to $4.75 \mathrm{~Hz}$ and $6.5 \mathrm{~Hz}$ and lasting for a total of $2.2 / 11 / 22 \mathrm{~s}$ and $1.5 / 7.5 / 15 \mathrm{~s}$. The characteristic time of the observed phenomena was assumed to be the characteristic time of the velocity variation. It was estimated as the ratio of a typical velocity to a typical acceleration and, in the worst case scenario, was found to be 15 times larger than the time between laser pulses. 

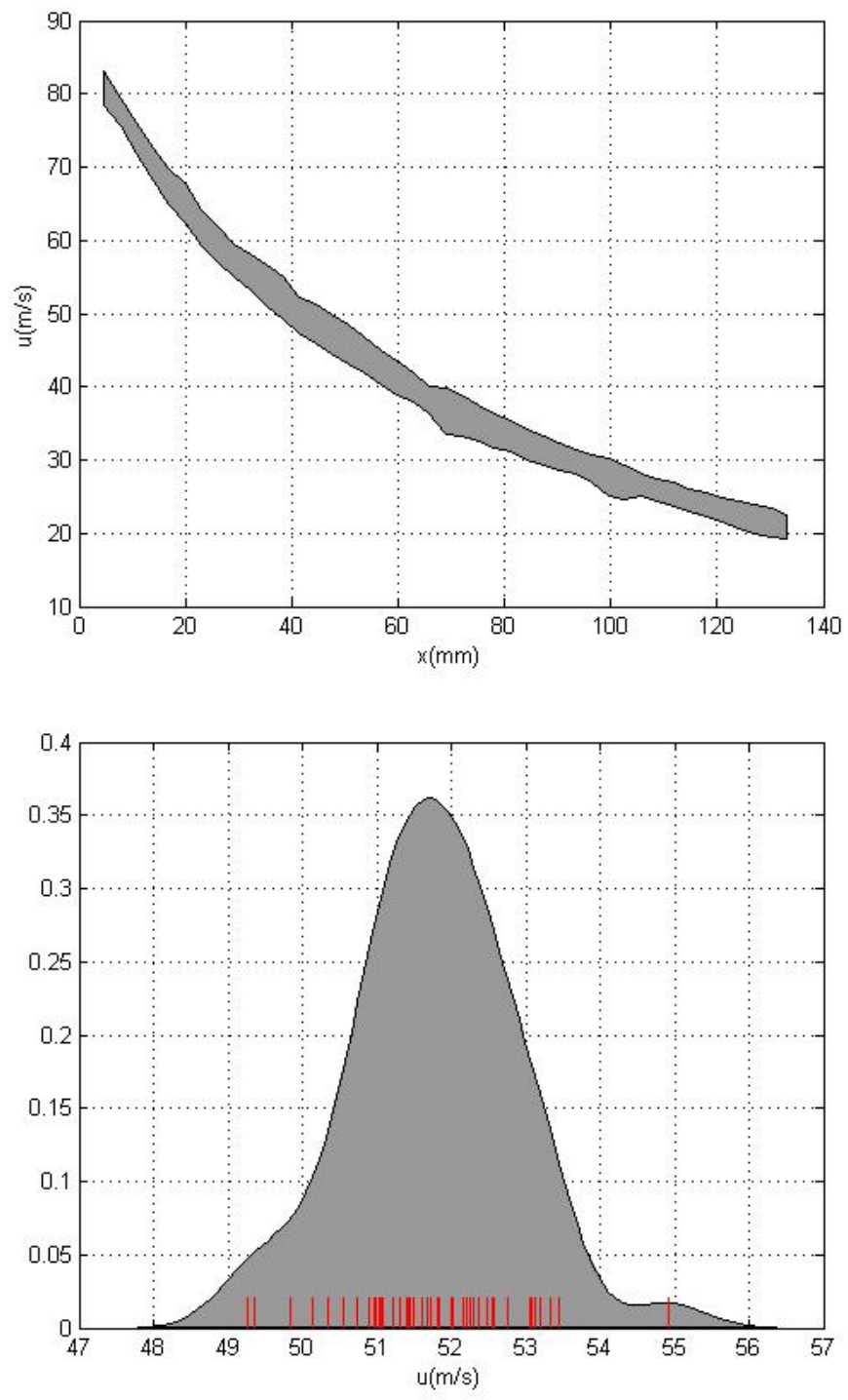

Figure 2.20: Top: dimensional uncertainty band with 50 different velocity profile measurements for the case of airfoil M1 and velocity equal to $90 \mathrm{~m} / \mathrm{s}$. Bottom: Kernel density estimate of the distribution of the 50 measurements corresponding to $\mathrm{x}$ $=40 \mathrm{~mm}$ (bottom). 


\subsubsection{Data Dispersion}

Regarding repeatability and dispersion of the results, each different case in the matrix of cases was repeated 50 times for M1 and M2 and 10 times for M3. It was found that dispersion was larger at the highest airfoil velocity with the larger airfoil. The spread of the measured velocity results obtained for this most critical case is presented in figure 2.20 (top). This picture is the outcome of 50 different velocity profiles measured under the same conditions. The maximum and minimum measured velocities at each spatial coordinate define the spread of the uncertainty band. It was found that the average spread was of the order of $3 \mathrm{~m} / \mathrm{s}$ for velocities in the range from $50 \mathrm{~m} / \mathrm{s}$ to $90 \mathrm{~m} / \mathrm{s}$. For each spatial coordinate, the 50 velocity samples followed a near Gaussian distribution as it could be observed in figure 2.20 (bottom) where the kernel density estimate of the 50 measurements corresponding to $\mathrm{x}=40 \mathrm{~mm}$ in figure 2.20 (top) is shown. The rug plot of the measurements is superimposed on the kernel density estimate plot.

\subsection{DATA PROCESSING SOFTWARE}

This section presents the methodology and specific software developed for the processing of high speed shadowgraph images taken during the experiments at the rotating rig facility. The large amount of data obtained from the experimental campaigns recommended the development of a specific application for processing purposes leading to the development of the so-called SITEA. SITEA is an application developed in a Matlab environment, designed for quick and efficient determination and processing of trajectories, deformations and breakup of droplets impinging on airfoils. The application is capable to track individual droplets giving the trajectory, and the maximum and minimum droplet diameters. In addition SITEA calculates velocities, accelerations and the non dimensional Reynolds, Bond, and Weber numbers along the stagnation line. A methodology to classify all the tests data obtained during the campaign was adopted to determine the input to the application. A defined storage format was also necessary to perform a later study. An image of the options that can be chosen during the analysis is shown in figure 2.21 .

\subsubsection{Pre-processing}

The large amount of information obtained during the test campaigns and the initial format of the recorded files, requires data pre-processing. Pre-processing consisted of the conversion from the video format to a format compatible with Matlab, PNG in this case, and the generation of an Excel file that identified and contained all the information related to each clip of images to be analyzed by the application. The relevant information for each clip was:

1. Test configuration: model used, model velocity and expected droplet diameter.

2. Frame identification: initial and final frame of the droplet tracking. The final frame corresponds to the moment just before droplet breakup or impingement on the model.

3. Identifying the frame in which model appears.

4. Location and generic name of the files. 


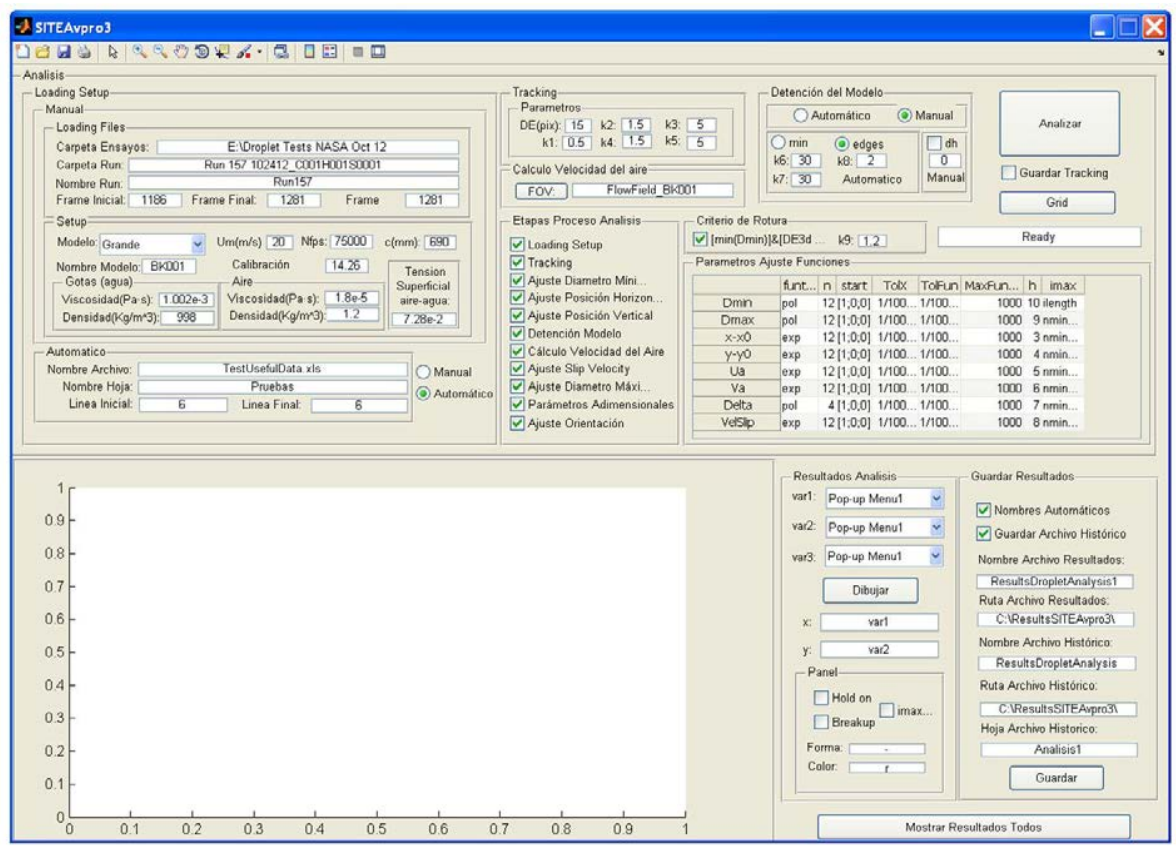

Figure 2.21: Last version of the SITEA in-house software

5. Number of droplets to analyze in each clip.

6. Acquisition configuration, including acquisition rate and magnification of the camera.

An Excell file was then generated containing all this information in a way that SITEA could collected it automatically.

\subsubsection{Processing}

SITEA was used to process the images recorded during the experiments. The way in which the videos are analyzed by SITEA is described in this subsection. The video processing consists of the analysis of each clip. The first frame of the clip is processed as follows:

1. Selection of the droplet to be tracked: Among all the droplets included in the first frame, one is selected to be tracked by marking on the frame a region of interest that includes only that droplet. An example can be observed in 2.22 .

2. Droplet detention: Images are converted to a binary image by a threshold selection, by using the Matlab sentences 'graythresh' and 'im2bw'. 'Graythresh' computes a global threshold using Otsu's Method [68] that converts an intensity image to a binary image with 'im2bw'. Otsu's method chooses the threshold to minimize the intraclass variance of the thresholded black and white pixels. This command works satisfactorily when the background of the image is homogeneous enough. Otherwise it is necessary to homogenize the background first. When the region of interest is relatively small, the background is usually sufficiently 


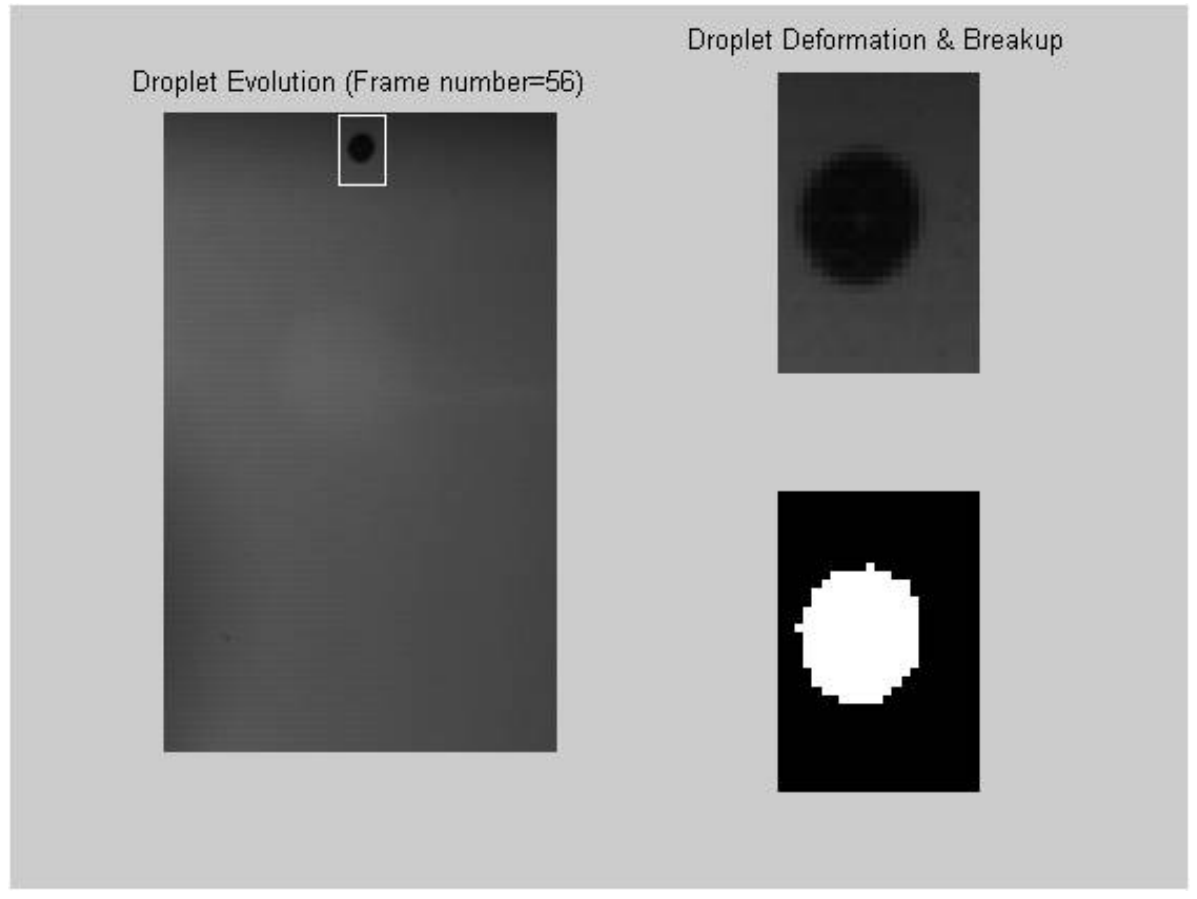

Figure 2.22: Example of the track of an individual droplet

homogeneous. This is the reason why only one droplet is tracked at a time. Attention must be paid to the contrast of the image so as to obtain the correct droplet shape in the binary image.

3. Image filtering: The binary image is filtered to discard glints and dust images. An sketch of the sequence of this process is shown in figure 2.23 , which includes:

- Image conversion to negative.

- Dust cleaning. Using the command 'bwareaopen' it is possible to eliminate white objects or dots owning areas smaller than a given number of pixels. In the cases studied, all the dust objects were correctly eliminated when the selected number of pixels was a $10 \%$ of the expected droplet area.

- Glint elimination. White holes in the center of the droplet due to light glints are removed using the command 'imfill'.

4. Acquisition of the droplet parameters: The command 'regionprops' is used to find the centroid position in the region of interest and the principal properties of the water drop, namely, droplet area, and minimum and maximum diameter. The droplet centroid coordinates, initially calculated in the local axes associated to the region of interest, are converted to frame axes. All calculated data are given in pixels and need to be converted to the international system of units using the camera magnification feature.

For the next frames, the region of interest is an area of the same size as the former but now centered at the position of the droplet in the previous frame. In a similar way 


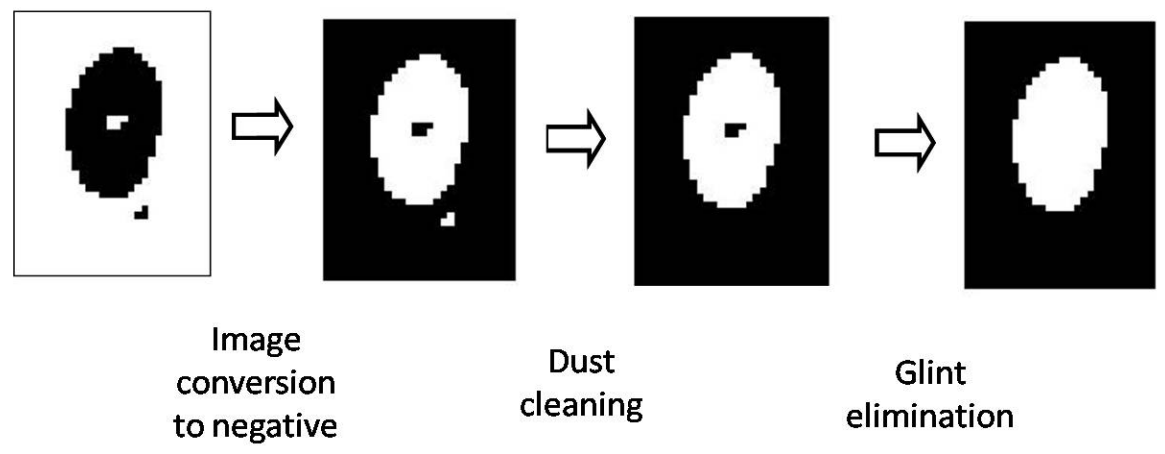

Figure 2.23: Image Processing for each frame during the analysis
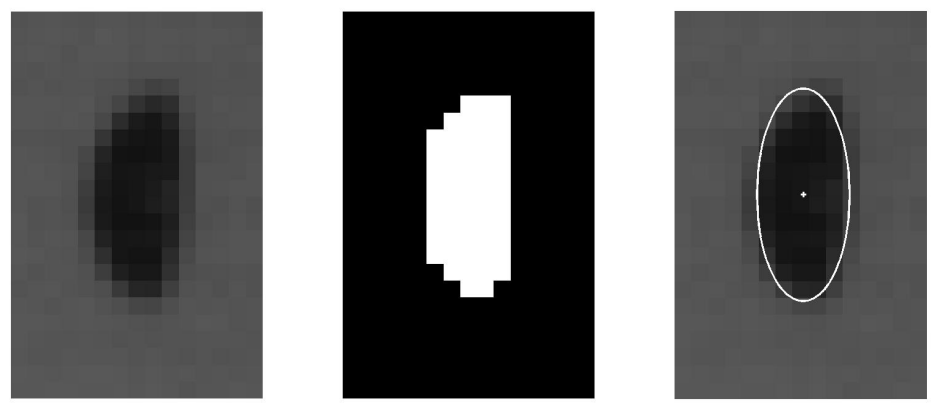

Figure 2.24: The three stages of image data analysis

each frame is analyzed. The area centroid determines the center of mass of the droplet and thus its trajectory.

Following the analysis of all frames, a change of the reference system is made from frame reference to model reference. The droplet velocity, the air velocity and the slip velocity are calculated. The breakup instant is also obtained by minimizing the fitted function of the droplet minimum diameter evolution following the breakup criteria used in reference [93].

\subsubsection{Uncertainty in the measurements}

During the processing the maximum and minimum diameter of the superimposed ellipse were obtained. The half-maximum 'a' and half-minimum ' $b$ ' diameters obtained during the processing are scalars specifying the length (in pixels) of the major and minor semi-axis of the ellipse that has the same normalized second central moments. A way to quantify the uncertainty of these measurements is to assume that the droplet deforms following the shape of an oblate spheroid whose maximum and minimum half-diameters are ' $a$ ' and ' $b$ ' respectively. Then, the volume of the spheroid can be readily computed. This, additionally, allows for the computation of the radius of an 


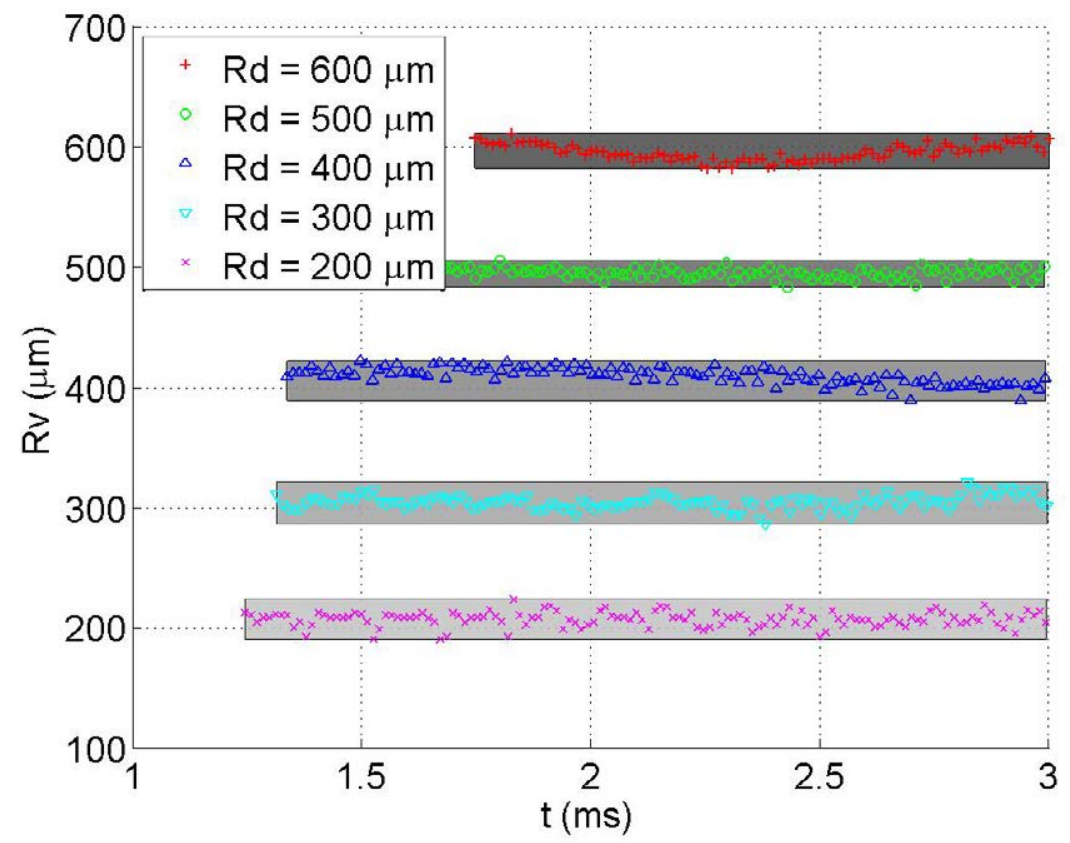

Figure 2.25: Evolution of Rv as a function of time for droplets of 200, 300, 400, 500 and $600 \mu \mathrm{m}$ of radius, for the model M1 moving at $80 \mathrm{~m} / \mathrm{s}$.

equivalent virtual spherical droplet that has the same volume. Then, the measurement uncertainty can be quantified plotting, as a function of time, this 'virtual' radius that should be constant (since the volume of the droplet remains constant). This 'virtual' droplet radius ' $\mathrm{Rv}$ ' is calculated for each frame as

$$
R_{v}=\sqrt[3]{a^{2} b}
$$

Figure 2.24 provides qualitative impression on the magnitude of the error associated to the estimate of both droplet maximum and minimum diameters. On the left, one original recorded image of the distorted droplet is presented; on the center, the binary image after cleaning dust and eliminating the glint is shown; and, finally, on the right, the original image with the superimposed computed ellipse is presented.

The measurements uncertainty bands associated to the computation of $\mathrm{Rv}$ are presented in figure 2.25 for five different cases for the M1 model airfoil at $80 \mathrm{~m} / \mathrm{s}$. The average Rv and its spread for these cases are: $596 \mu \mathrm{m} \pm 14 \mu \mathrm{m}( \pm 2.4 \%), 494 \mu \mathrm{m} \pm$ $11 \mu m( \pm 2.3 \%), 405 \mu m \pm 17 \mu m( \pm 4.1 \%), 304 \mu m \pm 17 \mu m( \pm 5.8 \%)$ and 207 $\mu m \pm 17 \mu m( \pm 8 \%)$. Since the pixel size is constant, the spread of the computed Rv grows as the initial droplet radius $\mathrm{Rd}$ decreases. In any case, the spread width varies between $5 \%$ for the largest droplets and $16 \%$ for the smallest ones which is deemed to be acceptable for deformation analysis purposes. 


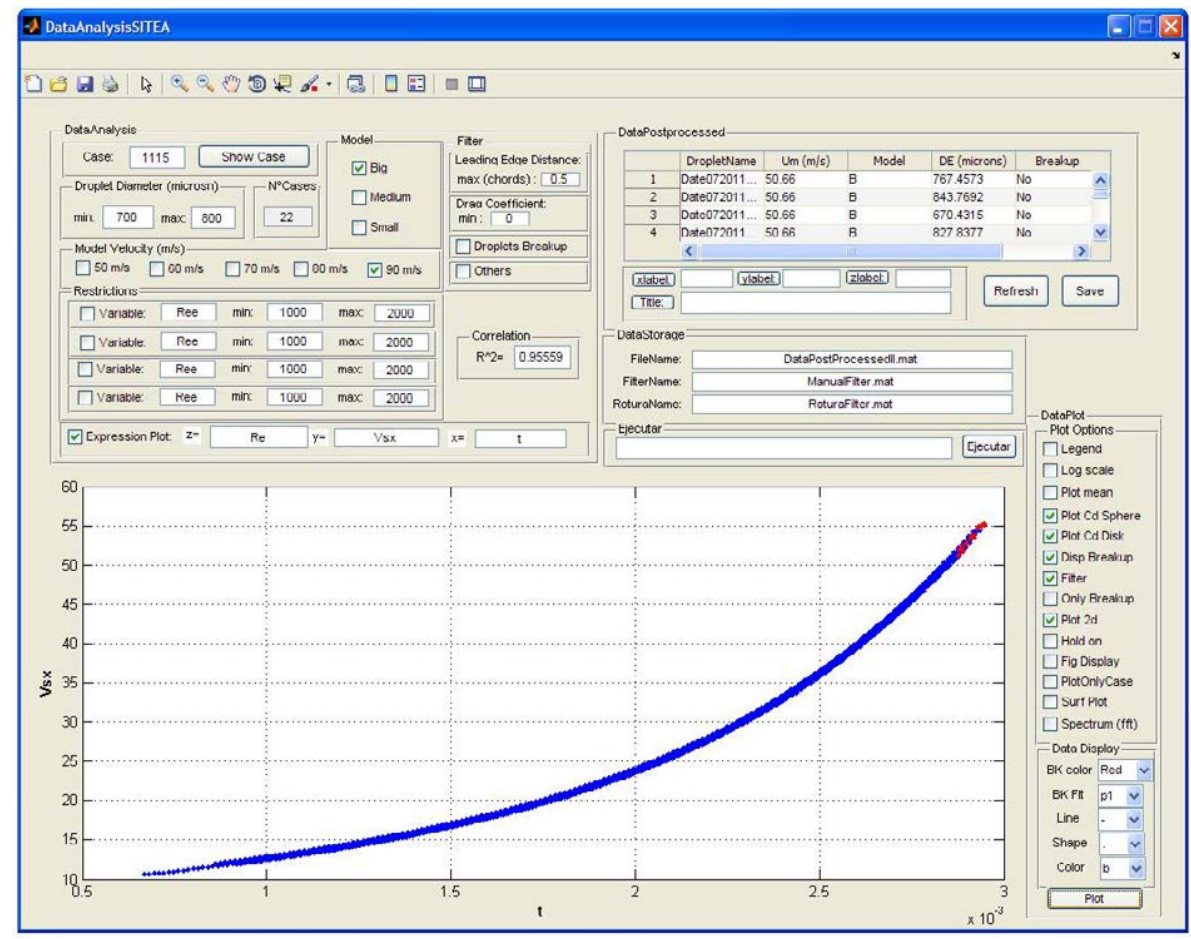

Figure 2.26: DataAnalysisSITEA application.

\subsubsection{Computational Time}

Running SITEA in a desktop computer (Pentium IV, 2800Mhz; 2Gb RAM), the application can process 250 frames in 20 seconds to obtain positions, areas and equivalent maximum and minimum droplet diameters, followed by an additional 30 seconds post processing period to obtain the water droplet velocity and acceleration.

\subsubsection{Visualizing the data: Data Analysis}

A total of 4902 droplets were analyzed for the first campaign. As a first approach to the data analyzed, another MATLAB application called 'DataAnalysisSITEA' was also developed to visualize the data. In this application the range of the desired droplets, the model velocity, and the model size is chosen as a filter of the data shown and the magnitudes obtained that wanted to be plotted are written. Finally, by pressing the button 'plot' the desired data is depicted. Additional options are also available, as seen in figure 2.26 . 


\section{Chapter 3}

\section{EXPERIMENTAL TESTING}

Preliminary results from the first experimental campaign on the deformation and breakup of water droplets in the vicinity of an incoming airfoil described in previous chapter is presented in this chapter. First of all, in the first section 3.1, the base flow field without droplets was characterized using a Particle Image Velocimetry technique. Then, in section 3.2, the rate of deformation of the droplets and the deformation and breakup processes were correlated to the parameters that defined the base flow. It was found that, within the range of tested experimental conditions, the favored breakup mechanism was the so-called 'bag and stamen' type. However, flow acceleration tended to anticipate the onset of this breakup mechanism as compared to previous experimental studies in which flow velocity was constant. This is important, for instance, for researchers that develop theoretical and numerical models of droplet-gas interaction for aircraft simulation purposes. Finally the main findings are summarized in the conclusion section 3.3.

The work presented in this chapter has been submitted, accepted and published in Aerospace Science and Technology [25] during the last year.

\subsection{BASE FLOW CHARACTERIZATION}

To relate the droplet deformation and breakup processes with the relevant governing flow parameters it was necessary to characterize the flow velocity field in the vicinity of incoming airfoils. This was done bearing in mind a one-way interaction approach, Iuliano [45], that means, in practice, that the base flow field was characterized without the presence of the droplets. A Particle Image Velocimetry (PIV) system was used for this purpose. The experimental setup was described in 2.2.2. First the PIV measurements are shown and then the next section is dedicated to the characterization of the base flow using these PIV measurements.

\subsubsection{PIV Measurements}

The average PIV results that were obtained are presented in figure 3.1. This figure 3.1 presents the velocity field, see figure 2.19 , in the planes $y=0$ and $z=0$. Figure 3.1(bottom) shows that the flow is reasonable constant in the span wise direction in the vicinity of the blunt airfoil leading edge. As explained in the previous section, PIV frames were obtained for different positions of the airfoils in their circular trajectories. 

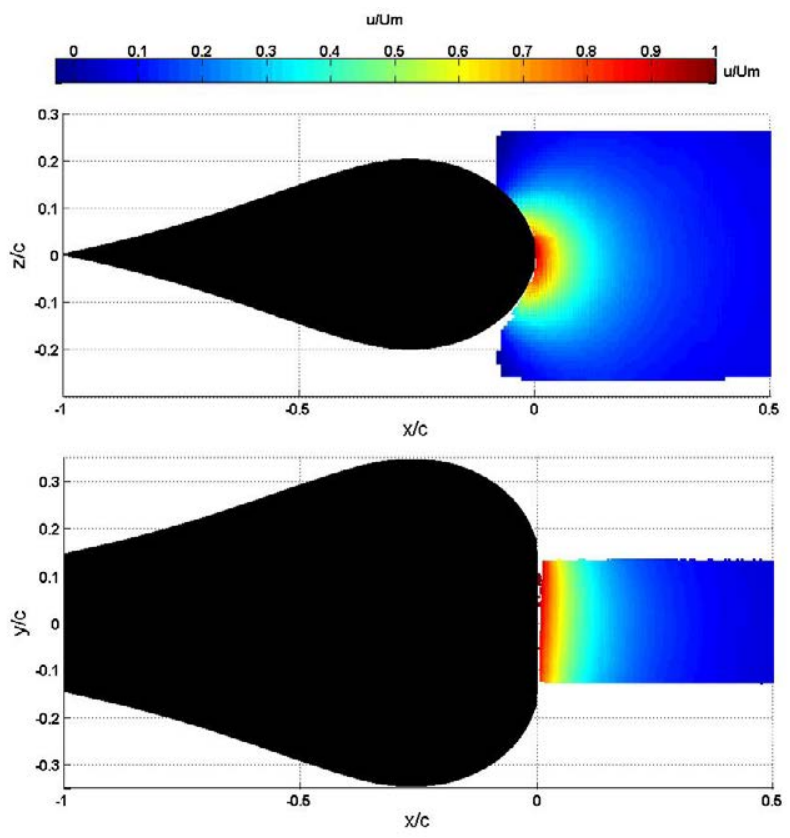

Figure 3.1: Horizontal $x$ velocity air flow field in the vicinity of the leading edge of the incoming airfoil. The $\mathrm{y}=0$ plane is plotted on top while the $\mathrm{z}=0$ plane is plotted on bottom

This allowed for the composition of the results so as to generate a single dimensionless velocity profile that is unique for all the experimental tests cases. This is possible because of the fact that the three airfoils shapes were similar and it was their actual dimensional size the parameter that was changed only. This single dimensionless velocity profile in the stagnation streamline of the airfoil is presented, after averaging, in figure 3.2 as a function of the dimensionless distance to the model (Um stands for the airfoil velocity).

\subsubsection{Characterization of the base flow}

In the windows of analysis during deformation and breakup tests, droplets covered a vertical distance of the order of 5 to $10 \mathrm{~mm}$. This distance was smaller than the airfoils leading edge radii $(103 \mathrm{~mm}, 70 \mathrm{~mm}$ and $30 \mathrm{~mm}$ respectively). Because of the pressure drag, the horizontal distance covered by the droplets during the tests was of $5 \mathrm{~mm}$ at most which was, again, smaller than the airfoils leading edge radii. Airfoil velocity was varied between $50 \mathrm{~m} / \mathrm{s}$ (Mach 0.14 ) and $90 \mathrm{~m} / \mathrm{s}$ (Mach 0.26) which means that some small compressibility effects were present.

The base flow, very close to the airfoil, as seen from the airfoil reference frame could be characterized as an approximate inviscid potential stagnation flow impinging on a flat surface; see Panton [69], Homann [37] and Weidman [98] for the 2D, axisymmetric and non-axisymmetric configurations respectively. The potential flow solution, regardless of whether the configuration is $2 \mathrm{D}$ axisymmetric or non-axisymmetric is characterized by a constant velocity deceleration along the stagnation streamline as the solid surface is approached. Specifically, $u=-b x$ where $\mathrm{u}$ is the stagnation streamline 


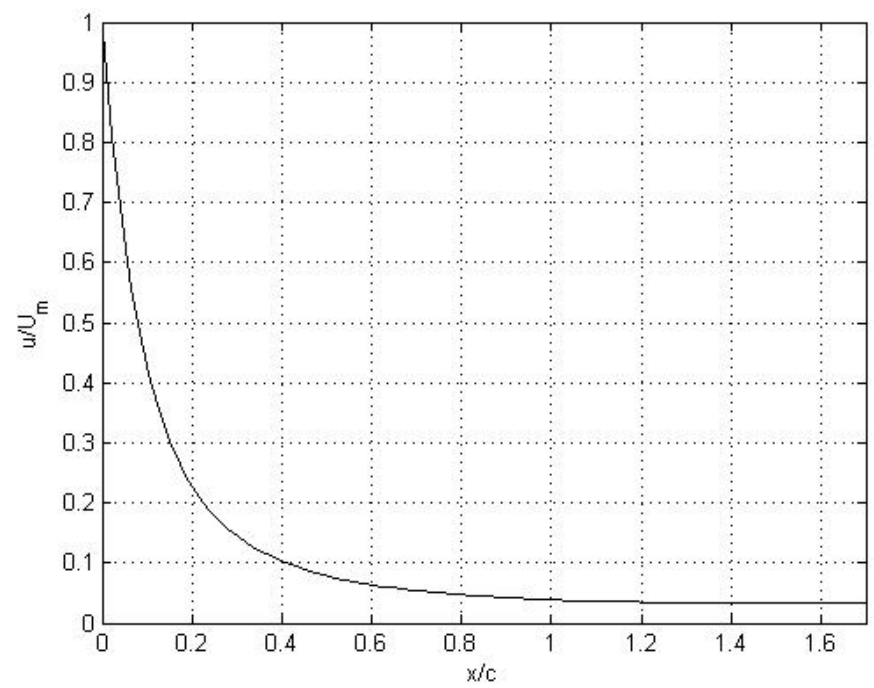

Figure 3.2: Dimensionless measured horizontal velocity profile in the stagnation stream-line of the airfoil as a function of the distance to the airfoil leading edge. Um stands for the maximum velocity: the airfoil velocity.

velocity, $\mathrm{x}$ is the distance to the stagnation point, and ' $\mathrm{b}$ ' is a constant that depends on the actual geometry. Obviously, the flow problem under consideration in this study is not potential but, for characterization purposes only, it could be characterized using the potential flow formulation where it more closely approaches the potential flow hypothesis; i.e.: in the region close to the stagnation point outside the boundary layer. Following this approach, the base flow could be characterized using two flow parameters: the airfoil velocity and the slope ' $b$ ' of the velocity profile along the stagnation streamline velocity in the vicinity of the airfoil stagnation region. This slope ' $b$ ' depends on both the airfoil velocity and leading edge radius of curvature. Figure 3.3 shows the measured dimensionless velocity profile and its linear approximation in the vicinity of the stagnation point for the larger airfoil.

The linear approximation plotted in dashed line in figure 3.3 can be written as

$$
\frac{u}{U_{m}}=1-8,28 \frac{x}{c}
$$

Or, equivalently,

$$
u-U_{m}=-\frac{8,28 U_{m}}{c} x
$$

that means that the slope ' $\mathrm{b}$ ' is $(8,28 U \mathrm{Um} / \mathrm{c}) \mathrm{s}^{-1}$. For the case of the larger airfoil $(\mathrm{c}=0.690 \mathrm{~m})$ at the highest velocity $(\mathrm{Um}=90 \mathrm{~m} / \mathrm{s})$ this value is $\mathrm{b}=1080 \mathrm{~s}^{-} 1$. Now, it is appropriate to compare this value of ' $b$ ' with similar cases reported in the literature. For example, White [99](Section 7.3.5 of his book) reports $\mathrm{b}=1694 s^{-1}$ for a circular 2-D cylinder having a radius equal to the leading edge radius of the larger airfoil $(0.103 \mathrm{~m})$ at the same flow velocity $(90 \mathrm{~m} / \mathrm{s})$. For a sphere in the same conditions $\mathrm{b}=1271 \mathrm{~s}^{-1}$, and for a flat nosed body $\mathrm{b}=685 \mathrm{~s}^{-1}$. The finite span configuration considered in the present study is 3-D and the leading edge region is flatter than the 


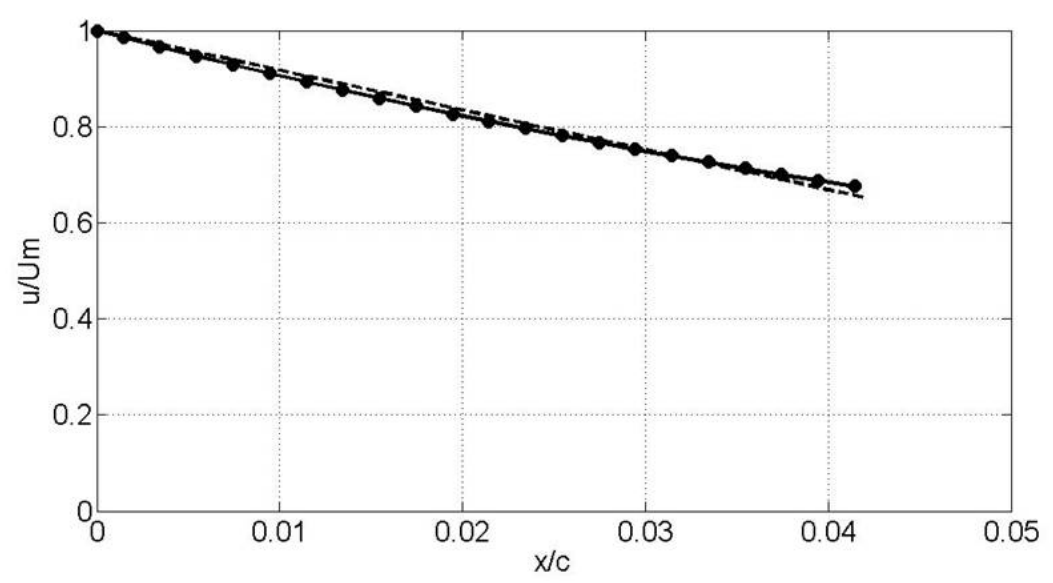

Figure 3.3: Dimensionless velocity profile (solid line) along the stagnation streamline in the vicinity $(x \rightarrow 0)$ of the stagnation point and its linear approximation (dashed line).

equivalent cylinder/sphere case; then, its associated value of ' $b$ ' should be intermediate between the sphere case and the flat nosed body which is indeed the case. The measured value of ' $\mathrm{b}$ ' $\left(1080 \mathrm{~s}^{-1}\right)$ can also be used to estimate the boundary layer thickness at the stagnation point. At $90 \mathrm{~m} / \mathrm{s}$, the Reynolds number based on the airfoil chord length is 3,900,000 so the flow is turbulent. However, a conservative laminar estimate can be done following, also, White [99] (Sections 3.8.1.1 and 3.8.1.2 of his book). In this case, it is found that the 'laminar' stagnation point boundary layer thickness is of the order of $0.3 \mathrm{~mm}$ which is much smaller than the leading edge radius $(103 \mathrm{~mm})$. Coming back to figure 3.3 , it could be observed that the validity of the potential flow solution extends up to about $30 \mathrm{~mm}(\mathrm{x} / \mathrm{c}=0.04)$ away from the stagnation point which is of the order of one third of the leading edge radius $(103 \mathrm{~mm})$.

\subsection{DROPLET DEFORMATION AND BREAKUP RE- SULTS}

Primary results from the first experimental campaign on droplets deformation and breakup experiments (described in chapter 2) are presented in this section and they are organized into three different sets (sets 1, 2 and 3) that involve eight different experimental cases. Many more different cases were measured and analyzed but it is believed that this selection sufficiently covers the spread of the experimental parameters that were varied and provides a generic overview of the observed phenomena. The definition of the sets and associated cases (identified by the airfoil model type, M, droplet typical diameter, Dd, and airfoil velocity, Um) is as follows:

- Experimental Set 1: Studies the effect of airfoil velocity for a fixed model and similar droplet sizes.

- Case 1: Model M1, Um=90m/s, Dd=0.782mm.

- Case 2: Model M1, Um=70m/s, Dd=0.804mm. 


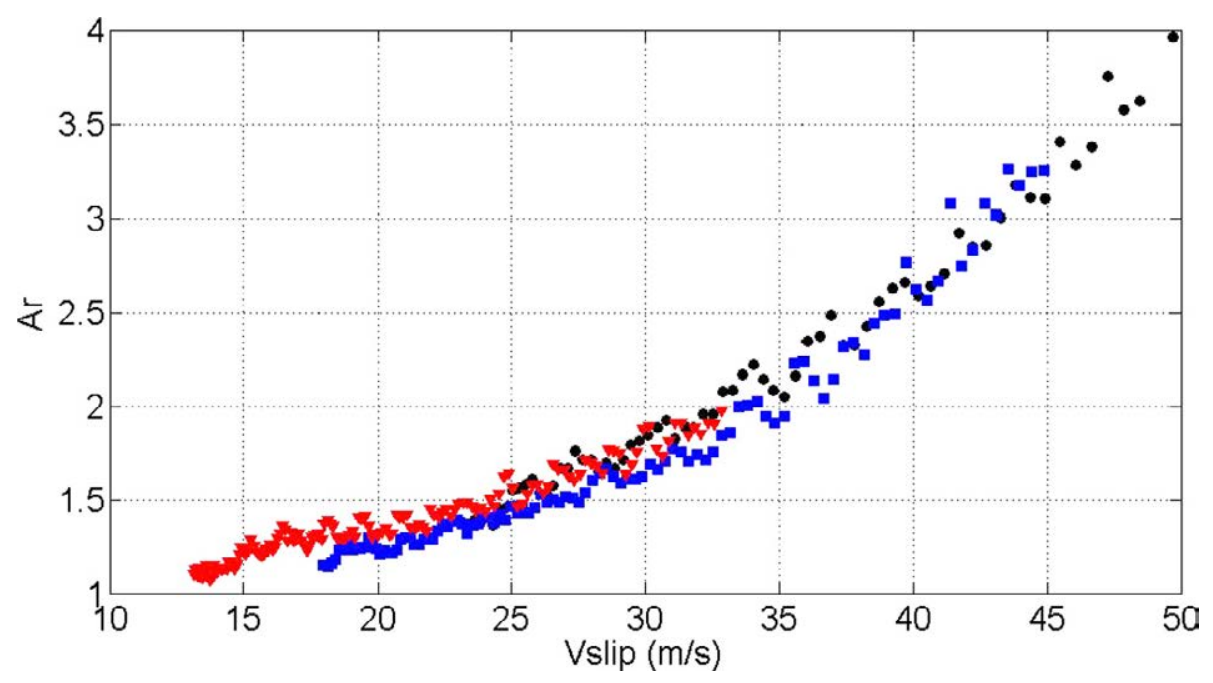

Figure 3.4: Droplet aspect ratio, Ar, as a function of the slip velocity, Vslip, for case 1 (black dots), case 2 (blue squares) and case 3 (red triangles).

- Case 3: Model M1, Um=50m/s, Dd=0.788mm.

- Experimental Set 2: Studies the effect of model size for the same velocity and similar droplet size.

- Case 1: Model M1, Um=90m/s, Dd=0.782mm.

- Case 4: Model M2, Um=90m/s, Dd=0.842mm.

- Case 5: Model M3, Um=90m/s, Dd=0.771mm.

- Experimental Set 3: Studies the effect of droplet size for the same velocity and airfoil model.

- Case 1: Model M1, Um=90m/s, Dd=0.782mm.

- Case 6: Model M1, Um=90m/s, Dd=0.354mm.

- Case 7: Model M1, Um=90m/s, Dd=0.580mm.

- Case 8: Model M1, Um=90m/s, Dd=1.056mm.

Case 1 appears in all three experimental sets because it could be considered as the reference case that helps to relate the different analysis and discussion of the results. It will be shown later that the slip velocity changes by a factor of the order of 2 during the residence time of the droplets in the analysis window where deformation and breakup take place. This means that there is an inherent coupling between the actual deformation and breakup processes and the velocity variation.It should be noted that in most of the previous experimental studies referenced to in the introduction chapter, flow velocity was constant during a time span long enough to define steady state Reynolds and Weber numbers. However, this was not the case in the present study. 


\subsubsection{Experimental Set 1}

Figure 3.4 presents the droplet aspect ratio, Ar, defined as the ratio of the droplet maximum versus minimum diameter, for the experimental set 1 (cases 1,2, and 3) as a function of the flow slip velocity past the droplet, Vslip. This Vslip was computed from the PIV base flow profiles and the actual droplet velocity obtained out of the high speed imaging system. The spatial window of measurement is the same for the three cases shown in 3.4. Specifically, this spatial window begins when the droplet is $124 \mathrm{~mm}$ away from the airfoil (horizontally) and ends when the droplet is $29 \mathrm{~mm}$ away (horizontally, again). The three cases have similar droplet diameter (about $0.8 \mathrm{~mm}$ ) and the same airfoil (M1). However, the airfoil velocities were different, which lead to different flow acceleration within the measurement window. In particular, from data shown in 3.2, a generic dimensionless relation between flow velocity and spatial coordinates for selfsimilar airfoil shapes can be written, which allows for computation of flow acceleration along the stagnation streamline. Then, the ' $u$ ' velocity at the stagnation streamline can be expressed as:

$$
\frac{u}{U_{m}}=\Phi\left(\frac{x}{c}\right)
$$

Since the actual distance ' $\mathrm{x}$ ' to the airfoil in the measurement window is a function of the initial distance ' $x_{0}$ ', the airfoil velocity ' $U_{m}$ ', and time ' $\mathrm{t}$ ', equation 3.2 can be re-written as:

$$
\frac{u}{U_{m}}=\Phi\left(\frac{x_{0}-U_{m} t}{c}\right)
$$

Which is derived with respect to time ' $t$ ' to get the acceleration ' $a$ '

$$
a=\frac{\mathrm{d} u}{\mathrm{~d} t}=-\frac{U_{m}^{2}}{c} \frac{\mathrm{d} \Phi}{\mathrm{d} \eta} \quad \eta=\frac{x_{0}-U_{m} t}{c}
$$

Now, this flow acceleration for the three cases 1, 2, and 3 is presented in Fig. 3.5 where it is apparent that acceleration profiles inside the measurement window are very different indeed (note that the airfoil chord length for these three cases is the same).

On one hand, it could be observed form figure 3.4 that, within the measurement uncertainty, the functional behavior of the aspect ratio versus the slip velocity appears to be independent of the flow acceleration that the droplet actually senses. In other words: this functional behavior would, in principle, suggest that the transient loading would not play a role in the process of droplet deformation. On the other hand, Luxford et al. [24] suggested that it is not only the transient acceleration loading but the residence time, $t_{r}$, of the droplet in the vicinity of the airfoil what influences droplet deformation. However, they did not provide the specific relationship between them. Then, following their line of thinking, the acceleration profiles shown in 3.5 have been scaled with the square of the associated residence time of the droplet inside the measurement window. The results are presented in 3.6 taking into account that the droplet residence times for cases 1,2 , and 3 were: $1.06 \mathrm{~ms}, 1.35 \mathrm{~ms}$, and $1.91 \mathrm{~ms}$ respectively.

As it could be observed in figure 3.6, all three curves collapse into a single one which suggests that as long as the functional relation/parameter (acceleration times the square of the residence time) is constant, the droplet deformation aspect ratio depends on the instantaneous slip velocity only (for the sake of brevity, the functional parameter will be called $\lambda$ hereafter). This is physically meaningful since larger transient acceleration loading tends to increase the droplet deformation aspect ratio but, at the 


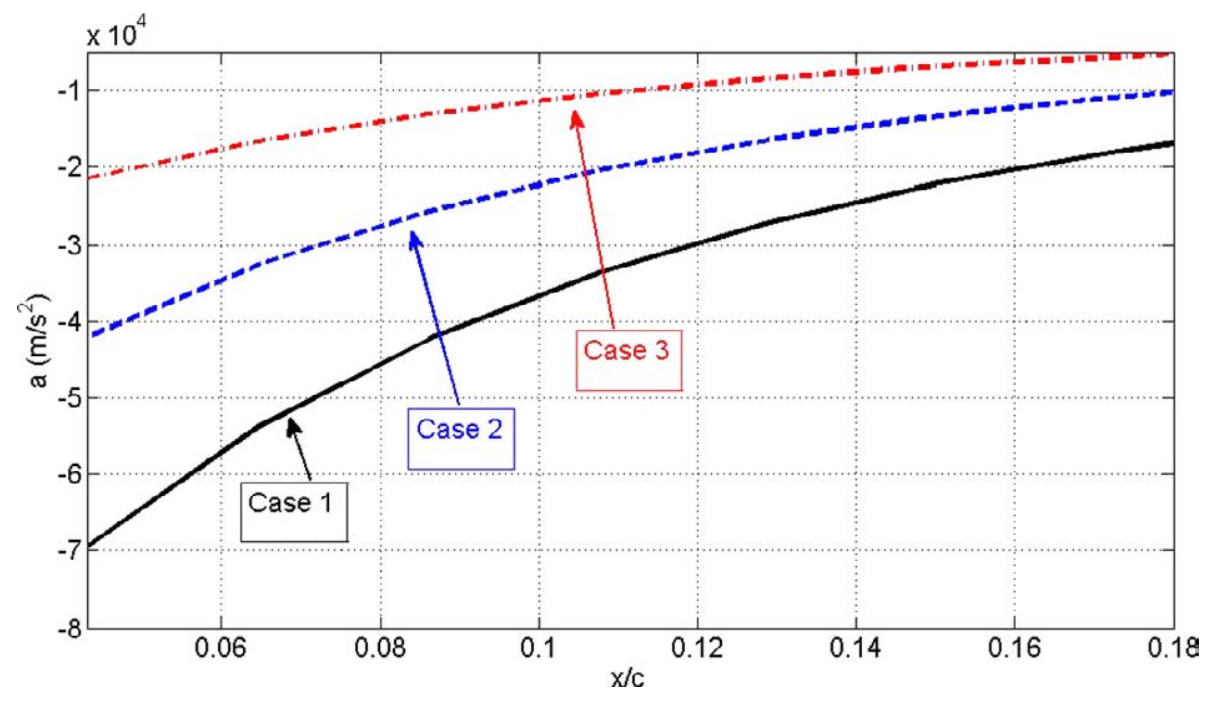

Figure 3.5: Flow acceleration profiles versus dimensionless distance to the airfoil stagna-tion point in the window of measurement for case 1 (solid black), case 2 (dashed blue), and case 3 (dashed dotted red).

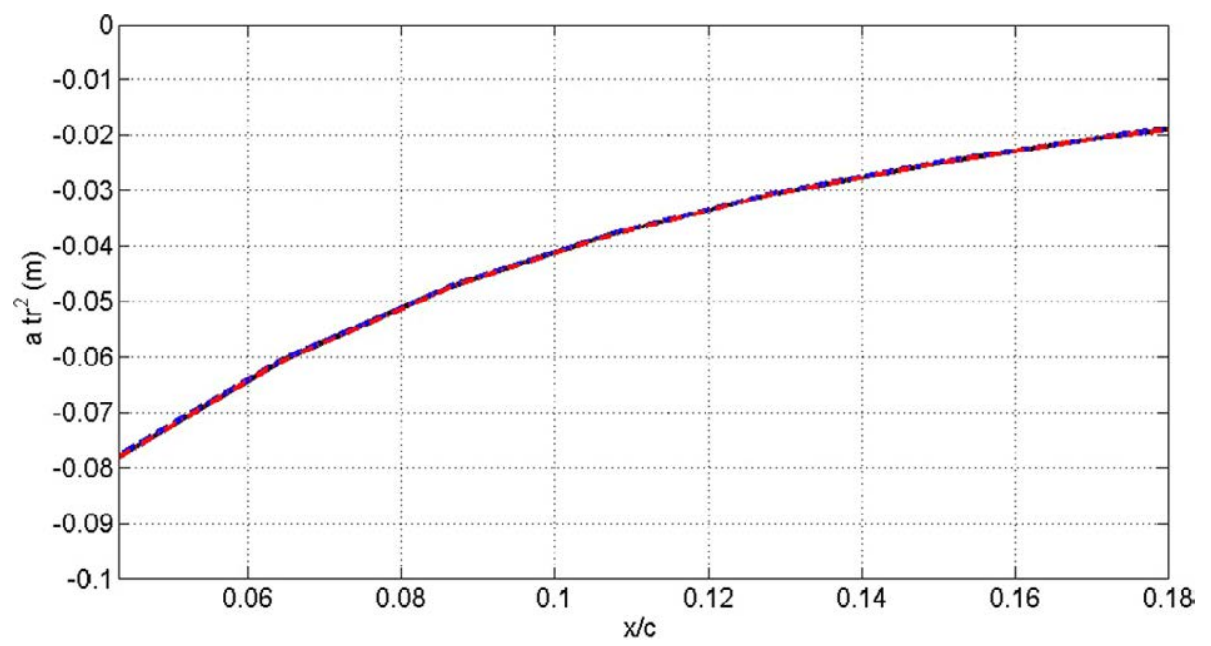

Figure 3.6: Flow acceleration times the square of the residence time as a function of the dimensionless distance to the airfoil stagnation point. Cases 1, 2, and 3 are plotted with the same key as in figure 3.5 and they collapse into a single curve. 
same time, shorter residence times do not allow this deformation to develop in full. The sequence of camera images associated to case 1 is presented in 3.7. This sequence can be qualitatively divided into a deformation process (up to $1.23 \mathrm{~ms}$ ) and the ensuing breakup process that follows. The deformation process is characterized by the fact that the droplet keeps a spheroid shape so that a maximum and minimum diameter can be meaningfully defined. This was done as described in chapter 2 by relating distances to pixels. Up to $1.23 \mathrm{~ms}$, the spheroid oblates significantly since the maximum diameters nearly doubles the original one while the minimum diameter almost halves the original one. That is, the aspect ratio of the spheroid at that precise instant is about 4. Beyond that point, the outer and center regions of the spheroid start to behave in a different way. The droplet losses its spheroid shape and a sharp spatial deformation is apparent in the sense that the droplet outer region moves away (in the flow stream wise direction) from the central region. Further on, this dislocation causes the droplet to break up. It is remarkable that at the time of $1.6 \mathrm{~ms}$ when the droplet outer region has completely broken and spread into a cloud, the central nucleus can still be clearly discerned. The instantaneous Weber number in the deformation regime (up to $1.23 \mathrm{~ms}$ ) changed in the range from 5 to 38 . This type of deformation and breakup is similar to the "bag and stamen breakup' regime described by Pilch and Erdman [73]. However, according to these authors, this 'bag and stamen' type of deformation and breakup occurs, for constant base flow velocity conditions, in the range of Weber numbers from 50 to 100. In fact, at lower Weber numbers, in the range from 12 to 50, Pilch and Erdman [73] report a different regime that they call 'bag breakup'. Then, it seems that the conditions of the present experiment (transient accelerating flow) tend to anticipate the onset of instabilities as compared with the equivalent constant base flow velocity experiments.

Now it is also relevant to compare the sequence presented in figure 3.7 with the sequences presented by Theofanous and $\mathrm{Li}$ [91] and Theofanous [90]. In those articles, the authors presented experimental evidence of two main deformation and aerobreakup regimes that they define as Rayleigh Taylor Piercing (RTP) and Shear Induced Entrainment (SIE) respectively. The RTP regime occurs at lower Weber numbers, typically below $10^{2}$, while the SIE regime occurs for Weber numbers above $10^{3}$. There is, also, a transitional region, where the Weber numbers range, typically, from $10^{2}$ to $10^{3}$, dominated by competition between Rayleigh Taylor waves leading to penetration, and shear induced convective effects that take droplet liquid away from the upstream stagnation point towards the droplet equator. An illustrative example of the RTP regime at a steady Weber number of 75 can be visualized in figures 5,13 and 14 of the article by Theofanous and $\mathrm{Li}[91]$. In these sequences, the droplets first deform towards an approximate spheroid shape and then undergo a fast breakup process characterized by a systematic topology disruption all over the droplet surface. In the present study, at an indicative Weber number in the range from 5 to 50 , the breakup process was different, see figure 3.7, and the behavior of the two distinct outer and center regions could be appreciated well into the breakup regime. It is to be noted that some operating parameters of the experiment were, also, very different. The static pressure and temperature of the already mentioned experiment by Theofanous and Li [91] were $45 \mathrm{~Pa}$ and 107 $\mathrm{K}$ respectively, while the present experiments were carried out at standard atmospheric conditions of $105 \mathrm{~Pa}$ and $295 \mathrm{~K}$. All these seem to suggest that the deformation and breakup process is rather sensitive to the flow conditions (either constant velocity or accelerating flow) and to the conditions of the experiment.

The sequence of images associated to cases 2 and 3 is presented in figure 3.8. It could be observed that in case 2 the same type of 'bag and stamen' breakup topology already shown in figure 3.7 is present here. 


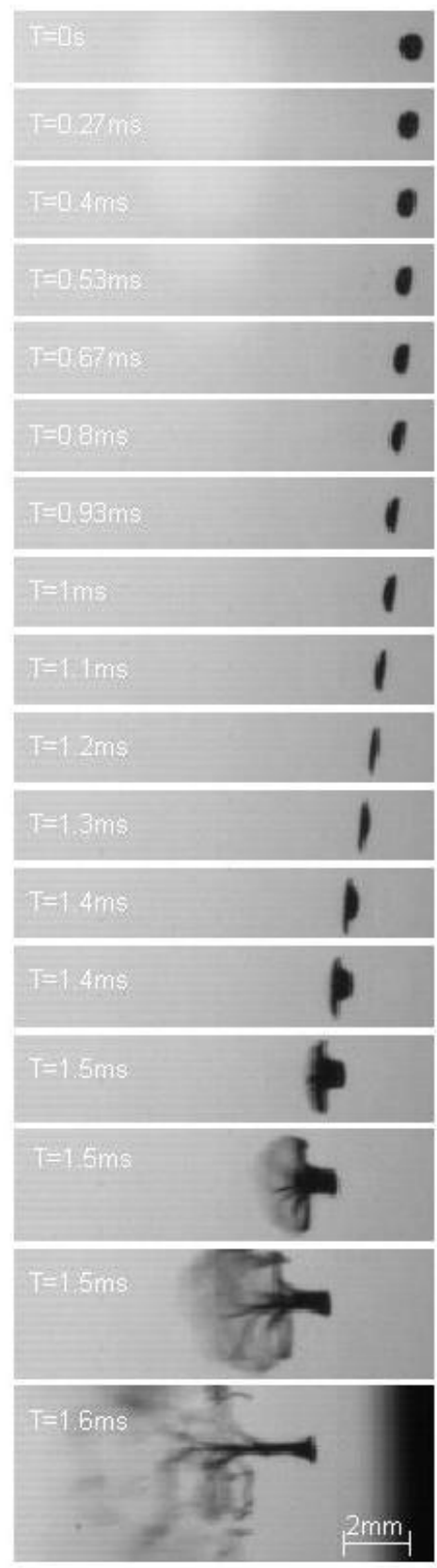

Figure 3.7: Sequence of images associated to the droplet deformation and breakup processes of case 1 . 


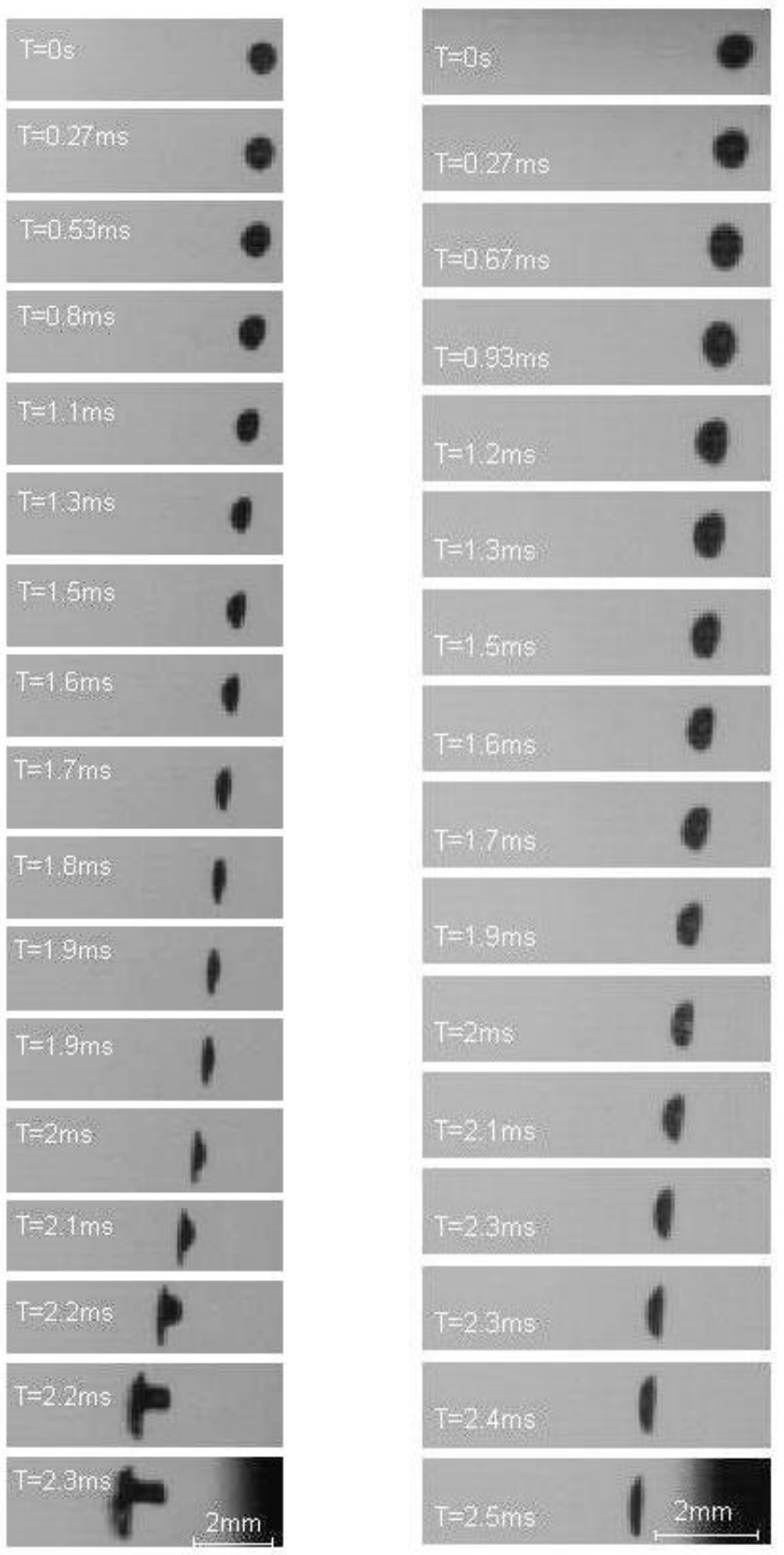

Figure 3.8: Sequence of frames for case 2 (left) and case 3(right). 


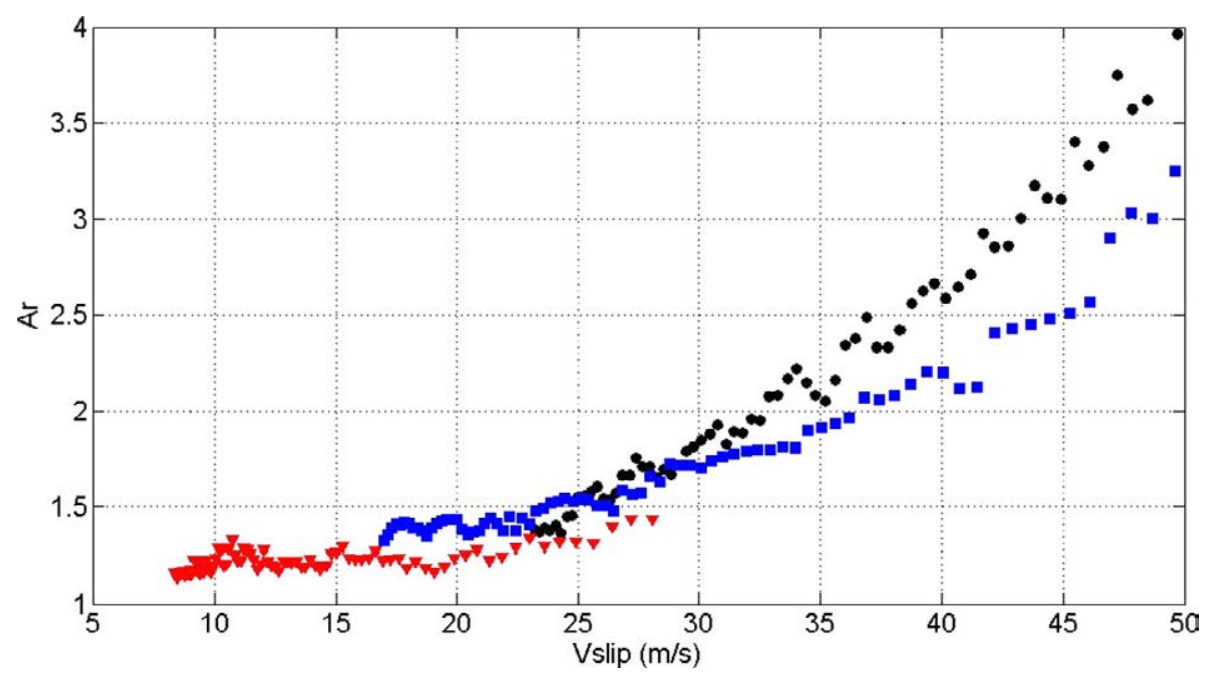

Figure 3.9: Droplet aspect ratio, Ar, as a function of the slip velocity, Vslip, for case 1 (black dots), case 4 (blue squares) and case 5 (red triangles).

\subsubsection{Experimental Set 2}

This experimental set 2 , that covers cases 1,4 , and 5 , is characterized by the same airfoil velocity $(90 \mathrm{~m} / \mathrm{s})$, similar droplet size (about $0.800 \mathrm{~mm}$ ), and different airfoil geometries which, in practice, leads to different values of the $\lambda$ functional parameter. Figure 3.9 is counterpart of 3.4 but containing the droplet aspect ratio results associated to cases 1, 4 and 5 as a function of the slip velocity. In this case, droplet deformation data does not collapse into a single curve/region as it happens in 3.6. Also, the slopes of the three data bases: case 1 (black circle), case 4 (blue square), and case 5 (red triangle) are different which suggests that a governing parameter has changed. According to the line of reasoning detailed in the previous subsection, the functional parameter could be $\lambda$. To test this hypothesis, the counterpart of 3.6 for cases 1,4 and 5 is presented in 3.10 where it could be observed that the acceleration function times the square of the residence time is indeed quite different which seems to support the ansatz that $\lambda$ is the governing functional parameter. The images associated to the deformation sequence of cases 4 and 5 are presented in figure 3.11 .

\subsubsection{Experimental Set 3}

In this experimental set the airfoil velocity was $90 \mathrm{~m} / \mathrm{s}$ and the airfoil was M1 (the largest). What changed was the droplet size since four different diameters were considered: $0.782 \mathrm{~mm}$ (case 1, black circles in figure 3.12), 0.364mm (case 6, red downward pointing triangles), $0.568 \mathrm{~mm}$ (case 7 , blue squares), and $1.075 \mathrm{~mm}$ (case 8 , green upward pointing triangles). The droplet residence time for these cases was the same (1.06 $\mathrm{ms}$ ) and, since the airfoil profile and velocity were the same, the functional parameter $\lambda$ was also the same. However, in this situation, it could be verified in figure 3.12 that the actual droplet aspect ratio for each slip velocity had a clear dependency on the droplet diameter. This might suggest that the actual deformation rate of the droplets could depend on two parameters. The first one, $\lambda$, contains information related to the flow external to the droplet, while the second would account for the physical characteristics 


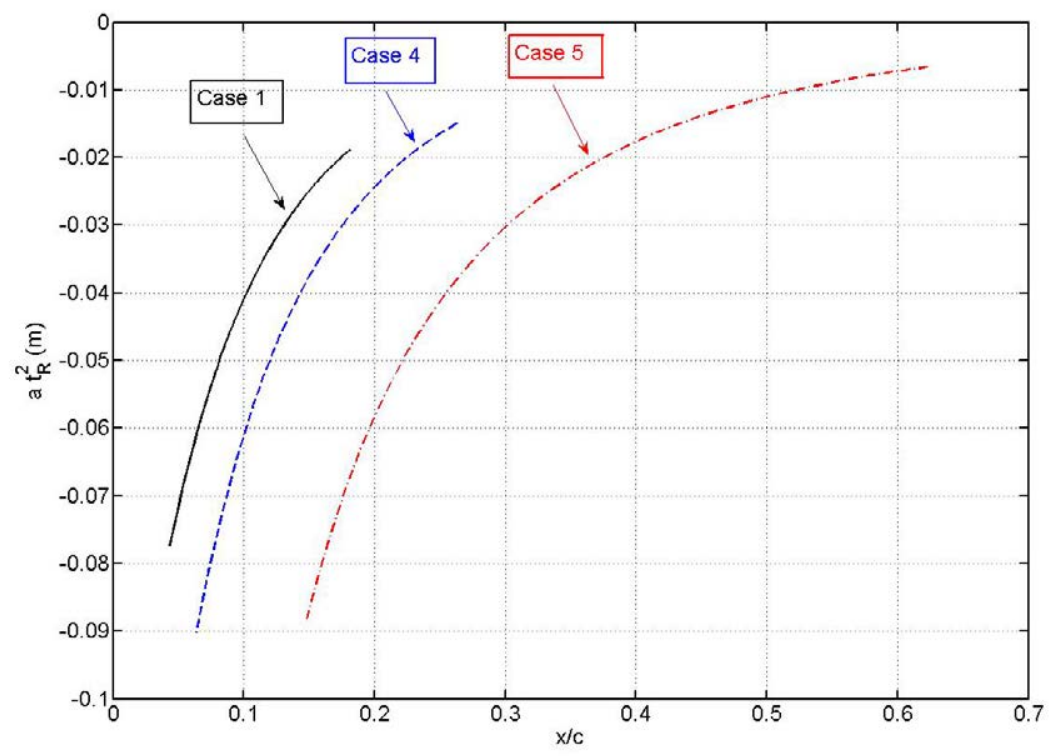

Figure 3.10: Counterpart of 3.6 for cases 1, 4 and 5. In this situation, the $\lambda$ profiles do not collapse into a single curve.

of the droplet itself. The substantiation of this hypothesis may require the coupling of two different sets of models, one for the flow field and another one for the droplet.

\subsection{CONCLUSIONS}

An experimental study has been presented on the problem of water droplet deformation and breakup in the vicinity of an incoming airfoil. The problem can be of interest in the context of understanding, predicting and simulating aircraft operations in a variety of flight conditions. The study considered three self-similar thick airfoils of different size (chords lengths: $0.690 \mathrm{~m}, 0.468 \mathrm{~m}$, and $0.199 \mathrm{~m}$ ), three different airfoil velocities $(90 \mathrm{~m} / \mathrm{s}, 70 \mathrm{~m} / \mathrm{s}$, and $50 \mathrm{~m} / \mathrm{s}$ ) and four different sizes of droplets (diameters of $0.354 \mathrm{~mm}, 0.580 \mathrm{~mm}, 0.782 \mathrm{~mm}$, and $1.056 \mathrm{~mm}$ ). The main conclusions can be stated as follows:

- The topology of the breakup mode in the cases where droplet breakup actually took place was of the 'bag and stamen' type. This could be relevant for researchers that develop numerical simulation codes aiming to predict flight operation under icing conditions.

- Transient effects (associated to the fact that the slip velocity past the droplets continuously increased) proved to play a relevant role. In particular, the results obtained suggest that the transient loading tends to anticipate significantly the onset of breakup as compared with steady state velocity experimental tests.

- It was found for droplets of the same size that, provided that the flow acceleration profile times the square of the residence time is constant (the $\lambda$ parameter), 


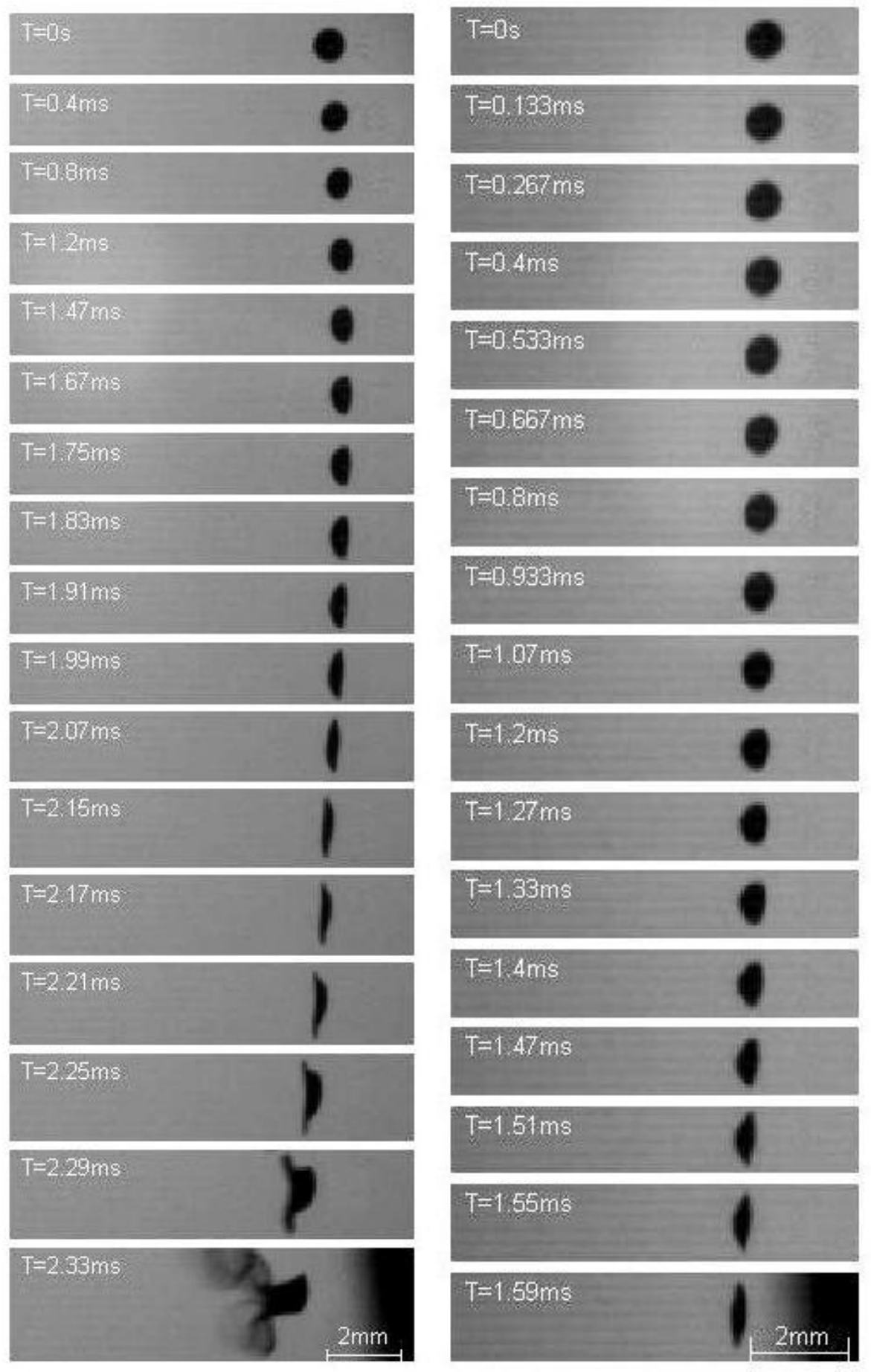

Figure 3.11: Sequence of frames for case 4(left) and case 5(right). 


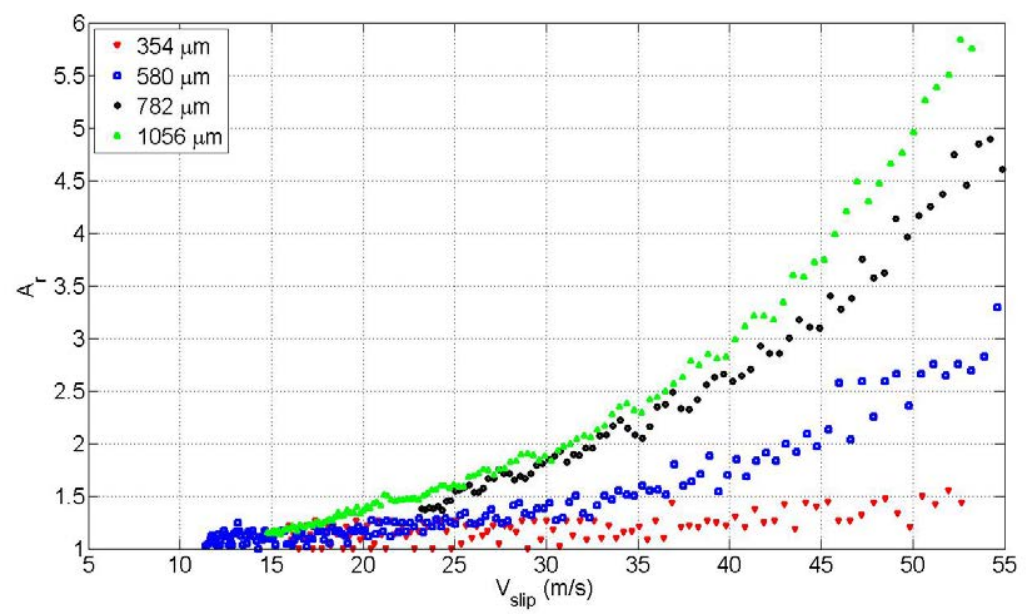

Figure 3.12: Droplet aspect ratio, Ar, as a function of the slip velocity, Vslip, for case 1 (black dots), case 6 (red downwards pointing triangles), case 7 (blue squares) and case 8 (green upwards pointing triangles). (For interpretation of the references to color in this figure legend, the reader is referred to the web version of this article.)

the droplet aspect ratio depends on the slip velocity only. This suggests that the behavior of droplet deformation evolution depends on a balance between the transient loading (the flow acceleration profile) and the droplet residence time (specifically, on the square of the residence time). This scaling law could be used as a first benchmark frame when developing theoretical models to predict droplet deformation and breakup modes.

- When considering the situation of a fixed airfoil model at a constant velocity, it was found that the actual droplet size had a marked influence on the droplet deformation ratio even if the $\lambda$ parameter was constant. This suggests that the problem might depend on, at least, two parameters: the first containing information of the flow field and the second related to the actual characteristics of the droplet.

- Regarding the experimental facility itself, it is to be said that a rotating arm unit appears to represent a reasonable compromise between compactness and capability to perform accurate experiments at an affordable cost. Obviously, this type of facility cannot address specific conditions, such as constant velocity flow fields, that can be attained in shock tube type test rigs, but it provide a suitable environment to address problems of technological interest in aerospace applications. 


\section{Chapter 4}

\section{DATA REDUCTION USING HOSVD}

The work presented in this chapter describes a data reduction method for the droplet deformation experiments carried out in the rotating arm facility described in chapter 2. The reduction method is based on the technique known as High Order Singular Value Decomposition (HOSVD). The idea is to find out whether, in this context, HOSVD allows for sufficient generalization of the results in a way that the outcome of new cases can be reasonably predicted with no need for further experiments. As in the experimental characterization in the previous chapter 3 , the data collected in the first experimental campaign described in chapter 2 were used for the study. Droplets were generated and allowed to cross the path of an incoming airfoil attached to a rotating arm. The high speed camera was used to record droplet deformation as a function of time. The flow field was characterized via Particle Image Velocimetry, as described in previous chapter 3. Airfoil velocity was varied between $50 \mathrm{~m} / \mathrm{s}$ to $90 \mathrm{~m} / \mathrm{s}$ and three different self-similar airfoils were used in the experiments with leading edge radii varying from $0.030 \mathrm{~m}$ to $0.103 \mathrm{~m}$. For this study, droplet radius was in the range from $200 \mu \mathrm{m}$ to $600 \mu \mathrm{m}$. The generated droplet deformation data was organized in the shape of a tensor having four dimensions: airfoil velocity, airfoil leading edge radius, droplet size, and time along droplet trajectory. The results obtained show that, in this problem, HOSVD can be reasonably used to densify the original experimental data tensor with acceptable accuracy. Thereby, allowing for the generation of reliable new information without having to perform additional experiments. This work was submitted to Experimental Thermal and Fluid Science and has been already published in 2016 [26].

Regarding the structure of this chapter, first an introduction to data reduction is made. Next, the HOSVD method is described and it is applied to seven application cases and discussed. Finally conclusions are given.

\subsection{INTRODUCTION TO DATA REDUCTION}

Data reduction is an important issue whenever experimental testing is involved. Its objectives are manifold; among others: to gather a better knowledge of the underlying physics, to generate scaling and/or design laws, and to further generalize results so as to uncover additional information without the need to perform additional experiments. Broadly speaking, data reduction methods can be classified into two main 
categories: those that rely on the model equations of the problem and those that do not. It is certainly much better to have a model that perform the data reduction in the light of a deeper physical insight. However, this is not always possible because of a number of reasons; among them physical model uncertainties, complex geometries and/or boundary conditions, and so on. Then, experimental researchers have developed a wide variety of methods that search for internal self-consistency and coherence of the collected data without reference to a specific physical model. Three examples, among many others, that involve data reduction in the context of the fluid equations of motion are those of Wang et al. [97], Kirk [51], and Vukoslacevic [94]. In the first article, Wang et al. [97] developed a data reduction method to predict the performance of a certain type of heat exchangers. The theoretical model in which their reduction method was based was zero-dimensional and did not involve local values of the variables. In the second article, Kirk [51] developed a thermo-fluid based data reduction scheme for hypervelocity experiments in a high speed wind tunnel at NASA. The method concentrated on modeling the error induced by different factors and involved the resolution of a computational fluid dynamics scheme. In the third article, Vukoslacevic [94] developed a data reduction method for a hot wire probe. The method involved the realization of a virtual experiment and the computation of local velocities and gradients. Regarding methods that do not directly involve physical model equations, Benay [7] has used a Levenberg algorithm for data reduction applied to a seven holes probe. One of the main objectives of the study was to reduce the size of the required data set needed for calibration purposes. Ni et al. [65] used a Hilbert-Huang transform to develop a simple, yet robust, data reduction method to determine hydraulic parameters in the Taipei basin. A Fourier transform (combined with a theoretical model) based data reduction method to evaluate local heat transfer coefficients has been proposed by Cossali [17]. A Wiener filtering technique for thermo-graphic data reduction of fin plate results aiming to estimate heat transfer coefficients has been proposed by Rainieri et al [76]. Although it may not be formally called data reduction, it is worth mentioning the study presented by Druault et al [21]. Specifically, the authors used a Proper Orthogonal Decomposition (POD) approach to interpolate in between consecutive PIV (Particle Image Velocimetry) time resolved frames. The fact that they were able to do so is linked to the property that POD modes have of efficiently and hierarchically storing dataset information patterns. Then, once these global modes were computed out of the finite set of PIV experimental frames, an algorithm was devised to fill in new information that was consistent with the POD global modes. In a context different from thermo-fluid-mechanics, Empirical Mode Decomposition (EMD) has been used by Rosas-Cholula et al [81] for headset data analysis and reduction purposes. Related to inverse analysis, Hwang et al [42] have used wind tunnel data in combination with a Kalman filter to achieve accurate identification of wind loads. A completely different approach to wind tunnel data reduction based on the use of a neural network has been proposed by English and Fricke [22]. Again, this may not be called data reduction on a strict sense, but the purpose was to generalize data so as to make accurate predictions of variables of interest. Aiming to generalization, Partridge and Keyhani [70] have published a generic data reduction method for interferometric data. Finally, although far apart from a thermofluid-mechanics area, it is interesting to quote the work of Nickerson and Sloan [66] that used a multivariate data reduction technique to generate both a reduced performance vector and a reduced set of decision variables in the context of a semiconductor production benchmarking study. 


\subsection{HOSVD METHOD DESCRIPTION}

HOSVD is an extension to n-dimensional tensors $(n>2)$ of the well known Singular Value Decomposition (SVD) technique that applies for two dimensional matrices only.

Specifically, the SVD of a $m_{1} \times m_{2}$ matrix $A$ consists of the decomposition $A=$ $U S V^{T}$ (the superscript T stands for the transpose). In this decomposition $U$ and $V$ are orthogonal matrices $\left(U U^{T}=U^{T} U=I\right.$, and $\left.V V^{T}=V^{T} V=I\right)$ and $S$ is a positive diagonal matrix whose $\mathrm{r}$ non-zero elements are called the singular values of $A$. Then, the rank of $A$ is $r$. Once the SVD decomposition is performed, the elements of $A$ can be written as:

$$
A_{i j}=\sum_{l=1}^{r} \delta_{l} U_{i l} V_{j l}
$$

In equation $4.1, \delta_{l}$ are the singular values of $A$. Also, for any given $l, U_{i l}$ and $V_{j l}$ are the elements of the $r$ column of $U$ and $V$. These are known as the left and right SVD modes. Note that each mode is one-dimensional because they are either the column or the row of matrices $U$ and $V^{T}$ respectively (for example, $U_{i 1}$ is the first column of $\mathrm{U})$. The singular values of $A\left(\delta_{1}, \ldots, \delta_{r}\right)$ in decomposition 4.1 are computed as the square root of the positive eigenvalues of the positive matrix $A A^{T}$ (that are the same as those of the matrix $A^{T} A$ ). The left and right modes are computed as the eigenvectors of matrices $A A^{T}$ and $A^{T} A$ respectively. When the singular values are organized into a decreasing order and decomposition 4.1 is truncated to $s<r$ terms, the truncation error (defined in terms of the Frobenius norm $\|A\|^{2}=\sum_{i, j}\left(A_{i j}\right)^{2}$ is:

$$
\| \text { error } \|=\left[\sum_{l=s+1}^{r}\left(\delta_{l}\right)^{2}\right]^{1 / 2}
$$

If error 4.2 is small, (i.e.: the singular values $\delta_{1}, \ldots, \delta_{r}$ exhibit a strong decay), a good approximation of matrix $A$ is:

$$
A_{i j} \sim \sum_{l=1}^{s} \delta_{l} U_{i l} V_{j l}
$$

For this decay to occur, it is necessary that the information contained in matrix $A$ is correlated. For example, if all numbers contained in $A$ are identical (let us say $A_{i, j}=1$ ) that represents the highest possible degree of correlation, $A$ can be expressed simply as the product of column vector (made up of ones) times a row vector (made up of ones) that means that $s=1$ in approximation 4.3. If $A_{i, j}$ is expressed as the product of a function $f(i)$ times a function $g(j)$ (that represents, also, a case with a high degree of correlation) it occurs again that $s=1$ since $A$ can be expressed as the product of column vector (containing $f(i)$ ) times a row vector (containing $g(j)$ ). In a more general case, reconstruction of the matrix $A$ would require the concurrence of several left and right modes, but the actual form of decomposition 4.1 ensures that $A$ is described as a series of successive global approximations whose hierarchy is governed by the singular values. In the case when the information contained in $A$ comes from some physical phenomenon governed by physical laws, the decay of the singular values $\delta_{1}, \ldots, \delta_{r}$ will certainly not be as sharp but it may allow, nevertheless, for some sensible truncation of the type described in 4.3 . 
Another interesting aspect of the SVD decomposition 4.1 is that once the singular values and the left and right eigenmodes are computed, it is straightforward to densify the data contained in A. This can be done very easily by interpolating inside the onedimensional modes $U_{i l}$ and $V_{j l}$ in decomposition 4.1. For example, assume that $A$ is $10 \times 10$ that means that each of the $r$ left SVD modes $U_{i l}(l=1, \ldots, 10)$ is made up of ten rows $(i=10)$. Then, each of them can be expanded to nineteen rows $(i=19)$, by merely interpolating in between every two values of two consecutive rows. Doing the same for the right modes and recomputing $A_{i j}$ via relation 4.1 generates a new $A_{i j}$ $(19 \times 19)$ matrix containing a much higher data density. It could be argued that this data densification process could be achieved, also, by local interpolation in the original $A_{i j}$ matrix. This is certainly true, but the advantages of using SVD for data densification purposes are twofold:

- The interpolation is one-dimensional inside the one-dimensional right and left SVD modes, and, therefore, more precise than a two-dimensional interpolation inside the original $A$ matrix (this advantage may seem marginal in this case, but it is critical in the case of multi-dimensional tensor to be described below when HOSVD is addressed).

- The new interpolated data is consistent with the global SVD modes, as opposed to a local interpolation that is consistent, only, with local information existing in the neighborhood of the newly generated data point.

A similar data densification approach can also be performed extrapolating out of the original matrix A. However, this process, that may yield information of interest, is not formally justified since the SVD modes span the interior of matrix $A$ only. In any case, when thinking of actual engineering related applications, it is worthwhile to evaluate the benefits of limited extrapolation because, in practical terms, it yields extra information (whatever its accuracy might be) at no extra cost.

HOSVD is the generic extension to multidimensional tensors $(n>2)$ of the SVD procedure already described. However, to keep the explanations intuitive, the following text will consider the particular case of a 3D tensor whose dimensions are $m_{1} \times m_{2} \times$ $m_{3}$.

The main difference between SVD and HOSVD is that in the later case, the 3D equivalent of the expansion of the type described in relation 4.1 would be the one described in relation 4.4 for which, unfortunately, there is no known procedure yet to find the solution, (de Lathauwer et al $[18,19]$ ). The reason is that the problem of finding the rank of higher-than-two order tensors ( $\mathrm{r}$ in decomposition 4.4) is still open.

$$
A_{i j k}=\sum_{l=1}^{r} S_{l} U_{i l} V_{j l} W_{k l}
$$

Then, instead of 4.4, many other less restrictive decompositions have been tried. Among them, HOSVD proposes the following alternative (Kolda [52]),

$$
A_{i j k}=\sum_{\eta=1}^{r_{1}} \sum_{\mu=1}^{r_{2}} \sum_{\xi=1}^{r_{3}} \Gamma_{\eta \mu \xi} U_{i \eta} V_{j \mu} W_{k \xi}
$$

In this decomposition, $\Gamma_{\eta \mu \xi}$ is called the core tensor and it is three dimensional. For each set of the three indexes $\eta, \mu$, and $\xi, U_{i \eta}, V_{j \mu}$ and $W_{k \xi}$ are called, in analogy to decomposition 4.1, the one-dimensional elements of the HOSVD modes. As it could be 
seen, the main difference between decompositions 4.1 and 4.5 is that $\delta_{l}$ in 4.1 depends on one index only, while $\Gamma_{\eta \mu \xi}$ in 4.5 depends on three indexes (n indexes in the general $\mathrm{n}$-dimensional case). Another important difference is that, as opposed to decomposition 4.4 , findings the unknowns in decomposition 4.5 is a well posed problem that can be readily solved. In particular, for each triplet of values $(\eta, \mu, \xi)$ the HOSVD modes are the orthonormal eigenvectors of the positive, symmetric matrices $B^{1}, B^{2}$ and $B^{3}$ defined as follows:

$$
\begin{aligned}
& B_{i l}^{1}=\sum_{j, k} A_{i j k} A_{l j k} \\
& B_{j l}^{2}=\sum_{i, k} A_{i j k} A_{i l k} \\
& B_{k l}^{3}=\sum_{i, j} A_{i j k} A_{i j l}
\end{aligned}
$$

Thus, the orthonormal HOSVD modes $U_{i \eta}, V_{j \mu}$ and $W_{k \xi}$ are given by

$$
\begin{array}{ll}
\sum_{l=1}^{m_{1}} B_{i l}^{1} U_{l \eta}=\alpha_{\eta}^{2} U_{i \eta}, & \eta=1 . . r_{1} \\
\sum_{l=1}^{m_{2}} B_{j l}^{2} V_{l \mu}=\beta_{\mu}^{2} V_{j \mu}, & \mu=1 . . r_{2} \\
\sum_{l=1}^{m_{3}} B_{k l}^{3} W_{l \xi}=\gamma_{\xi}^{2} W_{k \xi}, & \xi=1 . . r_{3}
\end{array}
$$

where (for $i=1,2,3) r_{i}\left(r_{i} \leq m_{i}\right)$ is the rank of $B^{i}$. The positive scalars $\alpha_{\eta}$, $\beta_{\mu}$, and $\gamma_{\xi}$ are the so-called high-order singular values of the decomposition. After the $(U, V, W)$ HOSVD modes have been calculated, the core tensor $\Gamma_{\eta \mu \xi}$ is obtained multiplying 4.5 by the $(i, j, k)$ component of the HOSVD modes and recalling that the HOSVD modes are orthonormal. Then:

$$
\Gamma_{\eta \mu \xi}=\sum_{i=1}^{m_{1}} \sum_{j=1}^{m_{2}} \sum_{k=1}^{m_{3}} A_{i j k} U_{i \eta} V_{j \mu} W_{k \xi}
$$

If decomposition 4.5 is truncated to: $s_{1} \leq r_{1}, s_{2} \leq r_{2}, s_{3} \leq r_{3}$, the original $A$ tensor is approximated as:

$$
A_{i j k} \approx \sum_{\eta=1}^{s_{1}} \sum_{\mu=1}^{s_{2}} \sum_{\xi=1}^{s_{3}} \Gamma_{\eta \mu \xi} U_{i \eta} V_{j \mu} W_{k \xi}
$$

The error of the truncated reconstruction, (de Lathauwer et al [19]) in terms of the Frobenius norm $\|A\|^{2}=\sum_{i, j, k}\left(A_{i j k}\right)^{2}$ is bounded by:

$$
\| \text { error } \| \leq \sqrt{\sum_{\eta=s_{1}+1}^{r_{1}}\left(\alpha_{\eta}\right)^{2}+\sum_{\mu=s_{2}+1}^{r_{2}}\left(\beta_{\mu}\right)^{2}+\sum_{\xi=s_{3}+1}^{r_{3}}\left(\gamma_{\xi}\right)^{2}}
$$

In this case, the information contained inside the original tensor can also be densified such as it was done in the case of SVD. Recall that to be consistent with tensor 
algebra, all modes have to be densified using the same criterion so that the global modes of both the densified and original tensors are the same. In the case of extrapolation outside the space spanned by the modes, caution must be exerted because the global modes of the newly densified tensor may differ from the (unknown) global modes of the original (tentatively enlarged) tensor.

\subsection{HOSVD ANALYSIS OF THE DATA}

The basic objective of this section is to ascertain whether it is possible, out of a limited set of droplet deformation experimental results, to densify the data set by means of generating new accurate information that could be used for different purposes. For instance, this data could be of interest for researchers developing numerical algorithms that simulate aircraft icing conditions $[3,35,45,47,80]$.

\subsubsection{Experimental Data Set}

As explained in detail in chapter 2, a first experimental campaign was carried out to obtain the time evolution of droplet deformation and breakup in a flow field generated by an incoming airfoil. Three Styrofoam airfoils of the same geometry but different size, labeled as M1, M2 and M3, were attached to the end of the rotating arm, resulting in translational velocities up to $90 \mathrm{~m} / \mathrm{s}$. Five velocities were selected during the experiments: $50 \mathrm{~m} / \mathrm{s}, 60 \mathrm{~m} / \mathrm{s}, 70 \mathrm{~m} / \mathrm{s}, 80 \mathrm{~m} / \mathrm{s}$ and $90 \mathrm{~m} / \mathrm{s}$. A stream of water droplets of radius in the range between 200 and $750 \mu \mathrm{m}$, generated by the monosized TSI MDG-10 droplet generator, was allowed to fall in the path of the incoming airfoil. A $2000 \mathrm{~W}$ xenon light source illuminated the droplets from the background and a high speed Photron SA-5 camera was used to capture droplet images at a rate of 75000 frames per second with a resolution of $192 \times 312$ pixels.

The data set on droplet deformation rate that has been compiled during the experimental campaign is four dimensional. Droplet deformation rate was defined as the minimum half-diameter divided by the droplet radius. A subset of the data of droplets of $200 \mu \mathrm{m}, 300 \mu \mathrm{m}, 400 \mu \mathrm{m}, 500 \mu \mathrm{m}$, and $600 \mu \mathrm{m}$ were used for this analysis. The axes of the four dimensional tensor where the information is stored are:

- axis 1 . Airfoil velocity (Um). Five cases: $50 \mathrm{~m} / \mathrm{s}, 60 \mathrm{~m} / \mathrm{s}, 70 \mathrm{~m} / \mathrm{s}, 80 \mathrm{~m} / \mathrm{s}$, and 90 $\mathrm{m} / \mathrm{s}$.

- axis 2. Airfoil leading edge radius $(\mathrm{Rc})$. Three cases: $0.030 \mathrm{~m}, 0.070 \mathrm{~m}$, and $0.103 \mathrm{~m}$.

- axis 3. Droplet radius (Rd). Five cases: $200 \mu m, 300 \mu m, 400 \mu m, 500 \mu m$, and $600 \mu \mathrm{m}$. Note that experiments were carried out for droplet radius up to 750 $\mu m$. However, because of consistency of the generated data, only droplets up to $600 \mu m$ in radius were considered.

- axis 4. Time (t). Twenty snapshots recorded for each case.

Then, the experimental data is stored in a tensor whose size is $5 \times 3 \times 5 \times 20$ that amounts to a total of 1500 pieces of information. It is important to note that not all cases that result from combining a certain airfoil velocity, airfoil leading edge radius and droplet size led to an actual droplet breakup in the experimental window. The reason is that, in some cases, the airfoil made an impact on the droplet before the droplet reached 

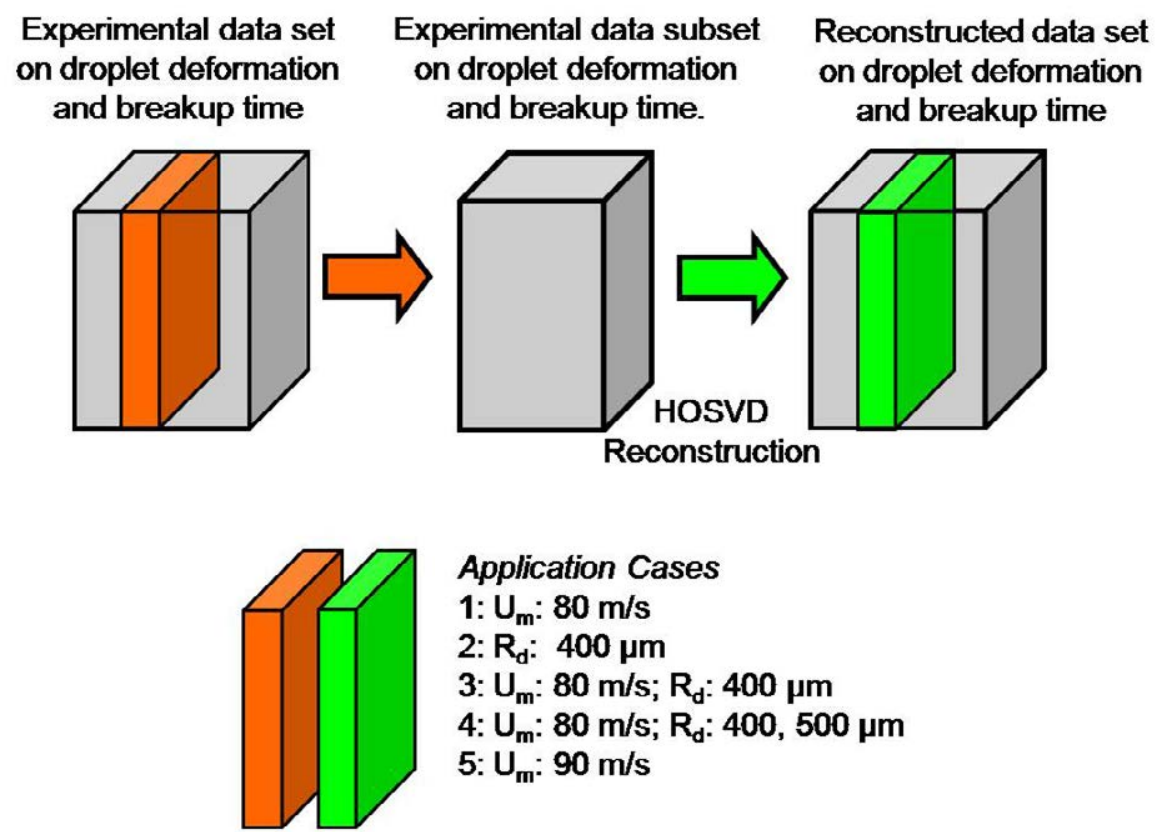

\section{Droplet deformation and breakup time comparison between experimental and reconstructed data}

Figure 4.1: Sketch of the process followed for the application cases.

the breakup stage. The first part of the analysis was performed with the data subset in which it was verified that droplet breakup occurred in all cases.

\subsubsection{Application Case 1}

The selected data subset on droplet deformation rate (tensor 1) in which breakup always occurs was:

- axis 1 . Airfoil velocity. Three cases: $70 \mathrm{~m} / \mathrm{s}, 80 \mathrm{~m} / \mathrm{s}$, and $90 \mathrm{~m} / \mathrm{s}$.

- axis 2. Airfoil leading edge radius. Two cases: $0.070 \mathrm{~m}$, and $0.103 \mathrm{~m}$.

- axis 3. Droplet radius. Four cases: $300 \mu \mathrm{m}, 400 \mu \mathrm{m}, 500 \mu \mathrm{m}$, and $600 \mu \mathrm{m}$.

- axis 4 . Time. Twenty time snapshots per each case.

To have a well conditioned tensor, airfoil velocities, airfoil leading edge radii, and droplet radii were rendered dimensionless dividing them by certain reference values. These reference values were the maximum airfoil velocity $\left(\mathrm{Um}_{\max }=90 \mathrm{~m} / \mathrm{s}\right)$, the largest leading edge radius $\left(\mathrm{Rc}_{\max }=0.103 \mathrm{~m}\right)$, and the largest droplet radius $\left(\mathrm{Rd}_{\max }=\right.$ $750 \mu \mathrm{m}$ ). Time, however, was rendered dimensionless using the actual breakup time, $t_{r}$, of each case. The reason is that $\mathrm{Um}_{\max }, \mathrm{Rc}_{\max }$, and $\mathrm{Rd}_{\max }$ are natural scales of the problem while $t_{r}$ depends on the specific conditions of every single experimental case. The breakup time was defined as the time of the breakup onset. It was actually 


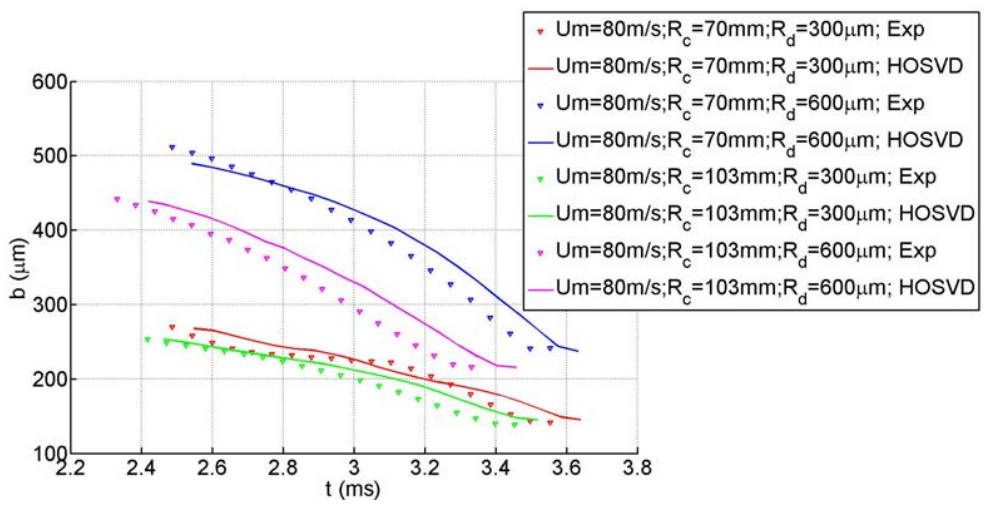

Figure 4.2: Comparison between reconstructed data (HOSVD in the legend) on droplet minimum half-diameter ' $b$ ' and actual experimental values (Exp in the legend) for four different cases.

defined as the moment when the assumption of an oblate spheroid type deformation does not longer hold, and the minimum diameter, which decreases all along the deformation process, starts to increase. This means that another additional tensor (tensor 2) containing the actual information on the breakup time is needed. This new tensor is three dimensional and contains $t_{r}$ as a function of the dimensionless airfoil velocity, airfoil leading edge radius and droplet radius. Now, the rationale for this application case 1 is as follows:

- The data corresponding to the velocity of $80 \mathrm{~m} / \mathrm{s}$ (axis 1) is dropped altogether from the experimental information (tensors 1 and 2). The actual objective is to replicate this data out of the remaining information. These two new tensors are named tensor 1' and tensor 2'.

- HOSVD is applied to tensor 2' and an interpolation on the modes is performed to recover the actual droplet breakup time that corresponds to a velocity of 80 $\mathrm{m} / \mathrm{s}$ (missing in tensor 2').

- HOSVD is applied to tensor 1'. New data are created for the missing velocity of $80 \mathrm{~ms}$, and droplet deformation rate is obtained as a function of the dimensionless time $\mathrm{t} / \mathrm{tr}$. Since $\operatorname{tr}$ was obtained in the previous step, the reconstructed droplet deformation versus physical time was predicted as well.

- HOSVD reconstructed data is compared to the original data. An sketch of the process followed is shown in figure 4.1.

Figure 4.2 shows the comparison between the thus reconstructed time dependent minimum half-diameter of the deformed droplet and the actual experimental values. Four cases are plotted simultaneously: 1) $\mathrm{Rc}=0.070 \mathrm{~m}, \mathrm{Rd}=300 \mu \mathrm{m}, 2) \mathrm{Rc}=0.070$ $\mathrm{m}, \mathrm{Rd}=600 \mu \mathrm{m}, 3) \mathrm{Rc}=0.103 \mathrm{~m}, \mathrm{Rd}=300 \mu \mathrm{m}, 4) \mathrm{Rc}=0.103 \mathrm{~m}, \mathrm{Rd}=600 \mu \mathrm{m}$. The airfoil velocity for all four cases was $80 \mathrm{~m} / \mathrm{s}$. The results presented in figure 4.2 are characterized by a reasonable agreement between predicted and actual experimental values. The maximum discrepancy in the four cases presented is $13.7 \%$, while the average discrepancy is $1.4 \%$. 
It is, also, illustrative to compare directly a series of experimental photographs with the superimposed droplet profiles (outlined in white color) computed out of the HOSVD reduction method. This is done for the case $\mathrm{Rd}=500 \mu \mathrm{m}, \mathrm{Rc}=0.103 \mathrm{~m}, \mathrm{Um}$ $=80 \mathrm{~m} / \mathrm{s}$ and the results are presented in figure 4.3 .

\subsubsection{Application Case 2}

Now, a similar approach is repeated but it is the data corresponding to droplet radius equal to $400 \mu \mathrm{m}$ that is dropped from the original tensors 1 and 2 . This data is reconstructed via HOSVD and the comparison between reconstructed and actual experimental data is presented in figure 4.4. The following cases have been selected for comparisons purposes: 1) $\mathrm{Um}=70 \mathrm{~m} / \mathrm{s}, \mathrm{Rc}=0.07 \mathrm{~m}, 2) \mathrm{Um}=70 \mathrm{~m} / \mathrm{s}, \mathrm{Rc}=0.103 \mathrm{~m}$, 3) $\mathrm{Um}=90 \mathrm{~m} / \mathrm{s}, \mathrm{Rc}=0.07 \mathrm{~m}, 4) \mathrm{Um}=90 \mathrm{~m} / \mathrm{s}, \mathrm{Rc}=0.103 \mathrm{~m}$. In this application case, the maximum and average discrepancies are $14.3 \%$ and $1.8 \%$.

\subsubsection{Application Case 3}

Now, two data sub-sets (instead of just one) are dropped from the two different axes of tensors 1 and 2: the data corresponding to airfoil velocity equal to $80 \mathrm{~m} / \mathrm{s}$ (axis 1 ) and droplet radius equal to $400 \mu \mathrm{m}$ (axis 3 ). In fact, this application case 3 results from combining the two previous cases. The comparison between the reconstructed and the actual experimental results is carried out for the following cases: 1 ) $\mathrm{Rc}=0.070 \mathrm{~m}$, and 2) $\mathrm{Rc}=0.103 \mathrm{~m}$, see figure 4.5 . In this application case, the maximum and average discrepancies are $15.8 \%$ and $2.2 \%$.

\subsubsection{Application Case 4}

Now, three data sub-sets are dropped from the original tensors 1 and 2: airfoil velocity equal to $80 \mathrm{~m} / \mathrm{s}$ in axis 1 , and droplet radius equal to $400 \mu \mathrm{m}$ and $500 \mu \mathrm{m}$ in axis 3. The comparison (four cases) between reconstructed and actual experimental results is presented in figure 4.6 for $\mathrm{Rc}=0.070 \mathrm{~m}$ and $\mathrm{Rc}=0.103 \mathrm{~m}$. In this application case, the maximum and average discrepancies are $28.3 \%$ and $3.3 \%$.

\subsubsection{Application Case 5}

This is a critical case because instead of interpolating inside the experimental data tensor, the objective is to extrapolate outside of it. HOSVD is not meant to extrapolate because projecting modes outside the space of parameters is not formally consistent with the method itself. However, it was deemed illustrative to see how the method performs outside its natural field of application. To do it, the data sub-set corresponding to the airfoil velocity of $90 \mathrm{~m} / \mathrm{s}$ is dropped from original tensors 1 and 2 . The comparison between reconstructed and actual experimental results is presented in figure 4.7. In this application case the maximum and average discrepancies are $28.3 \%$ and $4.5 \%$.

\subsubsection{Discussion on the application cases 1 to 5}

The reason why the comparisons presented in figures 4.2 to 4.7 show a reasonable agreement is that the data contained in the different tensors have a high degree of selfcorrelation. In a problem that depends on two parameters only, it is straightforward to 


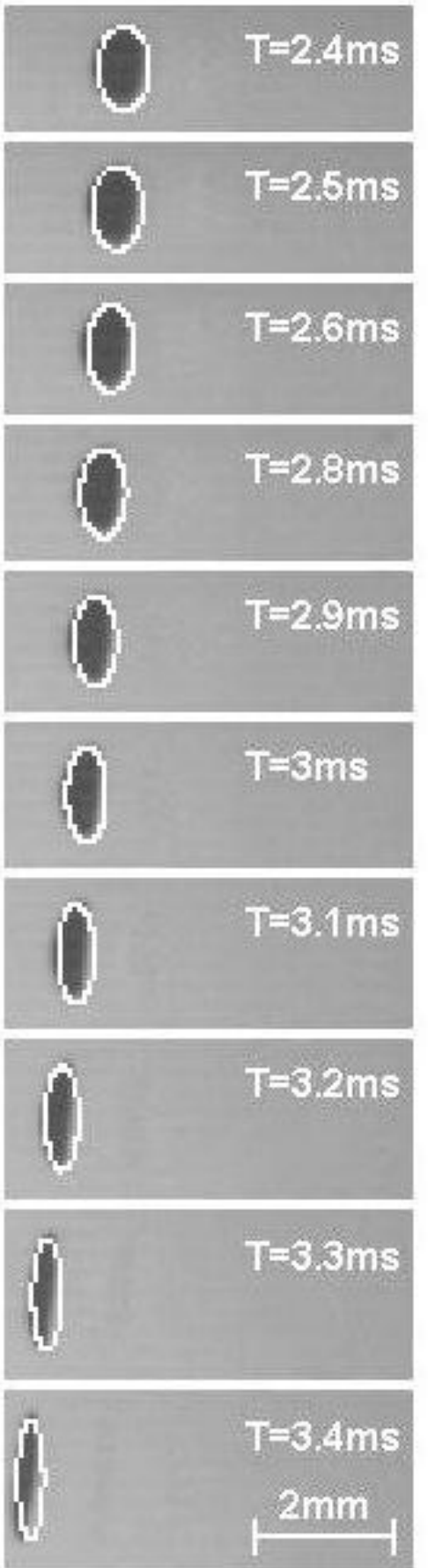

Figure 4.3: Comparison between a series of experimental pictures and the HOSVD computed droplet shape (outlined in white) for the case $\mathrm{Rd}=500 \mu \mathrm{m}, \mathrm{Rc}=0.103 \mathrm{~m}$ and $\mathrm{Um}=80 \mathrm{~m} / \mathrm{s}$. 


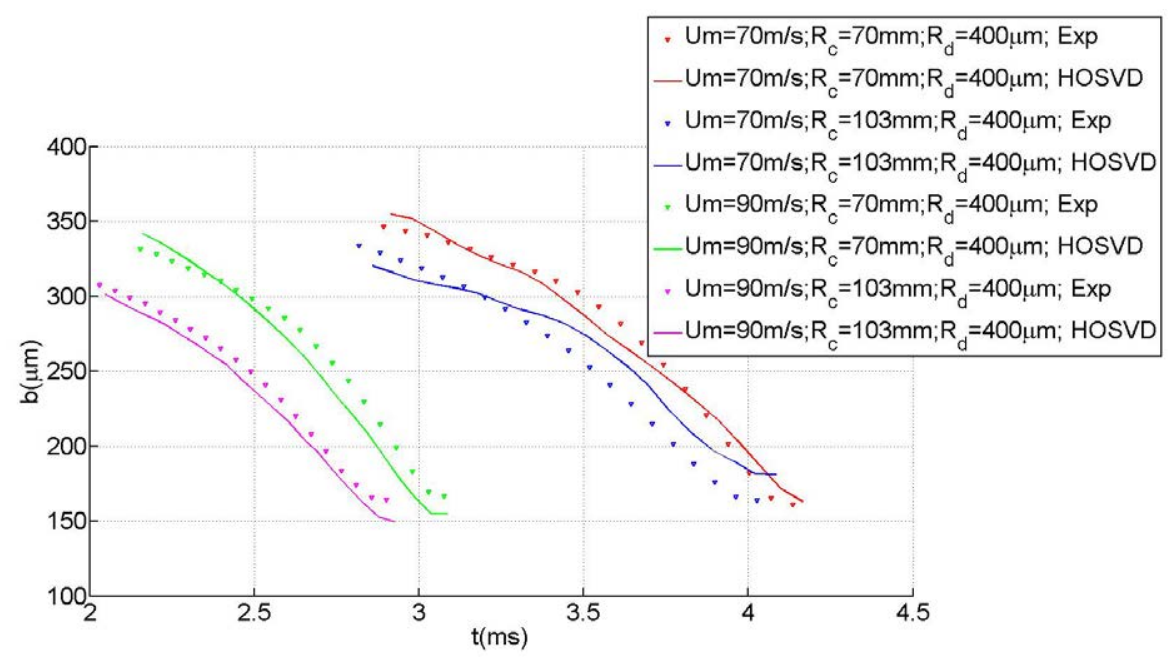

Figure 4.4: As in figure 4.2 but reconstructing a different data of the information tensor.

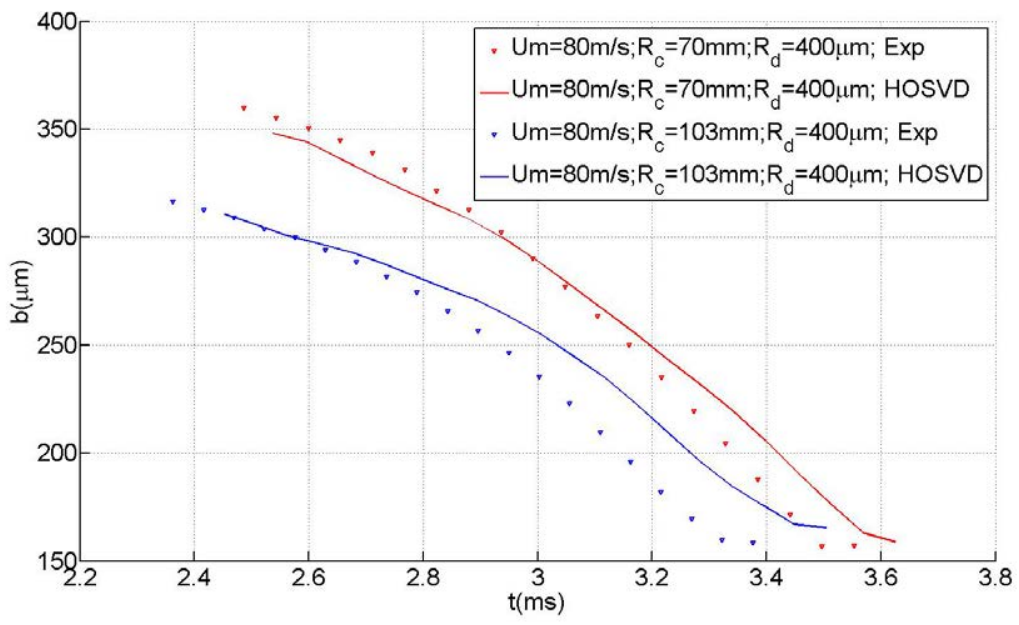

Figure 4.5: As in figure 4.2 but reconstructing two different data sub-sets of the information tensor. 


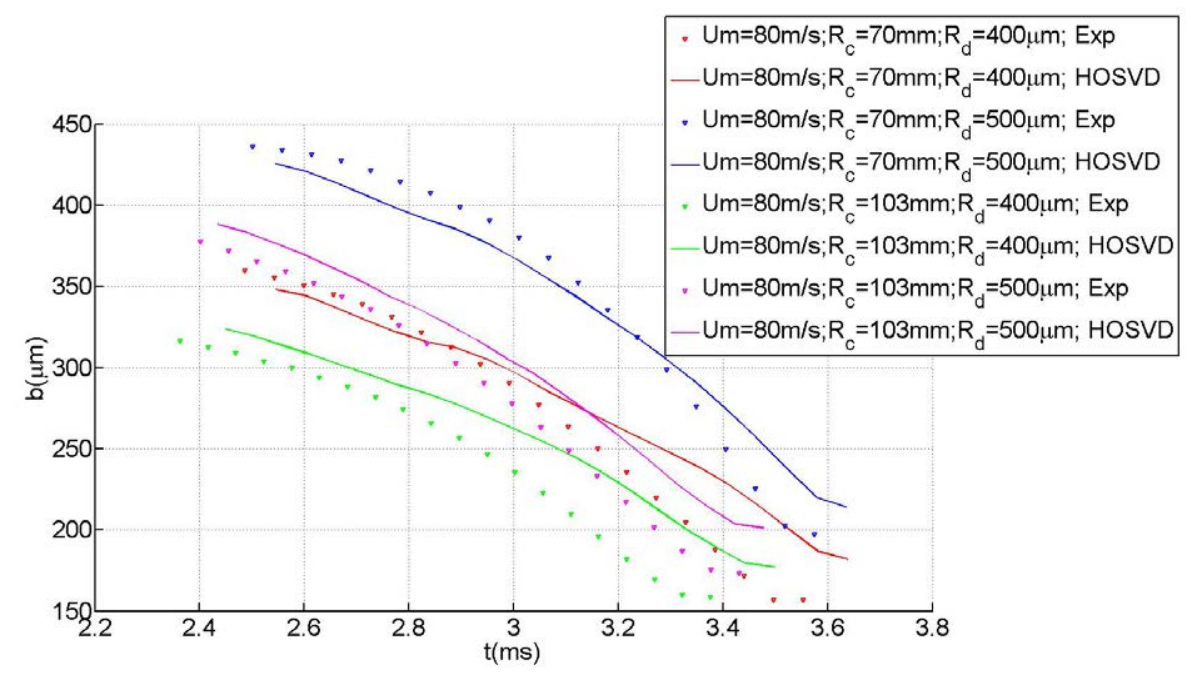

Figure 4.6: As in figure 4.2 but reconstructing three different data sub-sets of the information tensor.

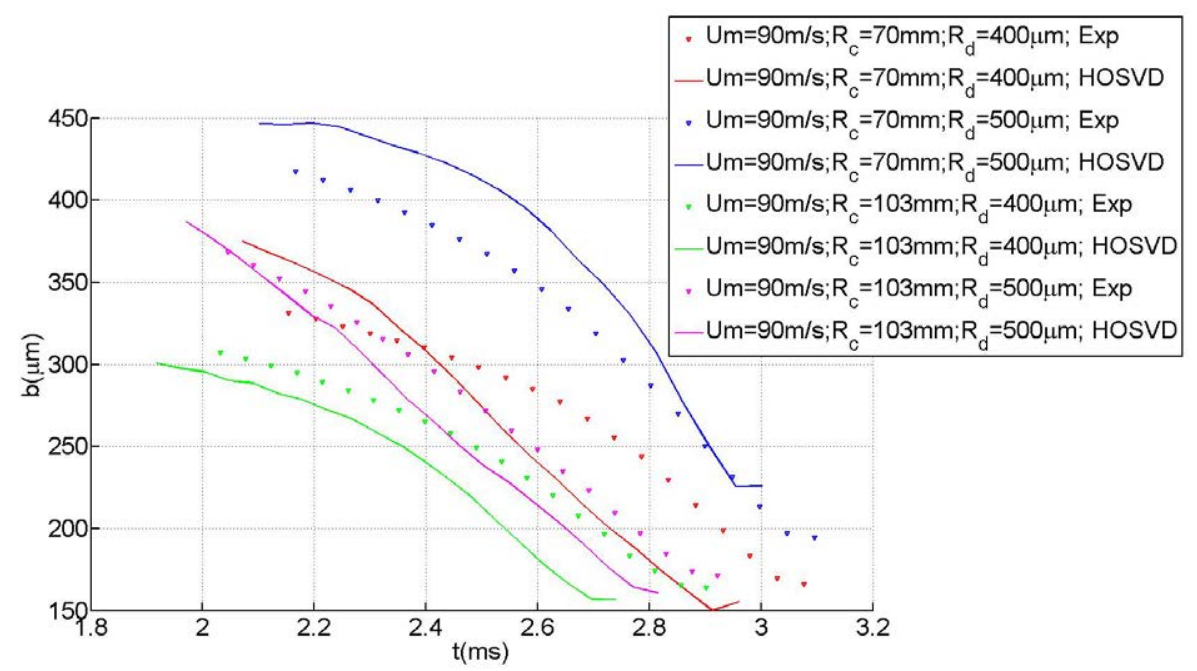

Figure 4.7: As in figure 4.2 but extrapolating one data sub-set out of the information tensor.) 


\begin{tabular}{ccccccc}
\hline \multicolumn{6}{c}{ Tensor 1. Bins per axis 3, 2, 4, 20 } \\
\hline \multicolumn{7}{c}{ Modes q1, q2, q3, and q4 retained per axis } \\
\hline A & 1 & 1 & 1 & 1 & 2.4 & 8.1 \\
B & 1 & 1 & 1 & 2 & 2.4 & 8.1 \\
C & 1 & 1 & 1 & 4 & 2.4 & 8.1 \\
\hline D & 2 & 2 & 2 & 1 & 2.0 & 8.1 \\
E & 2 & 2 & 2 & 2 & 1.5 & 5.2 \\
F & 2 & 2 & 2 & 4 & 1.4 & 5.0 \\
\hline G & 3 & 2 & 4 & 1 & 1.6 & 9.2 \\
H & 3 & 2 & 4 & 2 & 0.8 & 4.5 \\
I & 3 & 2 & 4 & 4 & 0.2 & 1.9 \\
J & 3 & 2 & 4 & 8 & 0.1 & 0.4 \\
\hline K & 2 & 2 & 4 & 4 & 0.9 & 3.8 \\
L & 2 & 2 & 4 & 20 & 0.9 & 3.8 \\
\hline M & 3 & 1 & 4 & 4 & 1.9 & 5.9 \\
N & 3 & 1 & 4 & 20 & 1.9 & 5.9 \\
\hline O & 3 & 2 & 1 & 20 & 2.3 & 8.9 \\
P & 3 & 2 & 2 & 20 & 1.2 & 4.3 \\
Q & 3 & 2 & 3 & 20 & 0.6 & 4.4 \\
\hline R & 3 & 2 & 3 & 4 & 0.7 & 4.4 \\
\hline & & & & & &
\end{tabular}

Table 4.1: Errors associated to the reconstruction of tensor 1 when retaining different numbers of HOSVD modes per axis.

visualize this self-correlation. In fact, it can be done by means of performing a Singular Value Decomposition (the two dimensional version of HOSVD) and plotting the singular values. If they decay quickly, the problem is highly self- correlated because most of the information is contained in a few modes. The case of HOSVD (dimensions higher than two) is different because, as explained in section 3, the singular values fill in a tensor themselves and visualizing the main diagonal of this tensor is not enough. Then, an alternative approach to see how much information is contained in the higher order modes consists of reconstructing the original information tensor retaining a limited number of modes and computing the error associated to this reconstruction. This is done in tables 4.1 and 4.2 for tensors 1 and 2 where for different combinations of retained modes both the average, $\epsilon_{a v}$, and the maximum, $\epsilon_{\max }$, reconstruction errors are shown. These errors are defined as:

$$
\begin{gathered}
\epsilon_{a v}=\text { mean }\left(\mid \text { tensor }_{\text {original }}-\text { tensor }_{\text {reconstructed }} \mid\right) \\
\epsilon_{\max }=\max \left(\mid \text { tensor }{ }_{\text {original }}-\text { tensor }_{\text {reconstructed }} \mid\right)
\end{gathered}
$$

The following aspects could be considered when analyzing the results presented in table 4.1:

- If only a few q1, q2 and q3 modes are retained, reconstruction errors depend weakly on the actual number of time-frames taken (axis 4 of tensor 1). This could be observed, for example, in cases $\mathrm{A}, \mathrm{B}$, and $\mathrm{C}$ where doubling the number of $\mathrm{q} 4$ modes retained does not influence the mean and maximum reconstruction errors. 
This could also be observed in cases $\mathrm{K}$ and $\mathrm{L}$ and, also in cases $\mathrm{M}$ and N. Cases $\mathrm{D}, \mathrm{E}$, and $\mathrm{F}$ are slightly different because even though that mean error remains at a similar level, the maximum error decreases when doubling the number of $\mathrm{q} 4$ modes.

- When the number of retained q1, q2, and q3 modes increases (cases G, H, I, and $\mathbf{J}$ ), that means that more information is exposed, the number of retained $\mathrm{q} 4$ modes indeed influences the reconstruction errors. Sequential doubling of retained q4 modes ( 1 in case G, 2 in case H, 4 in case I, and 8 in case J) leads to average and maximum reconstruction errors of $0.1 \%$ and $0.4 \%$ respectively. This, for all practical experimental purposes (that always involve some degree of uncertainty), implies that all relevant information is retained when choosing three q1 modes, two q2 modes, four q3 modes, and eight q4 modes.

- Cases H and I suggest, also, that only a limited number of q4 modes are needed to reconstruct with a reasonable degree of accuracy the desired information. In fact, retaining only two q4 modes lead the average reconstruction error below the $1 \%$ threshold, while four q4 modes lower the maximum error below the $2 \%$ level.

- Finally, case O, P, and Q show the sensitivity of the reconstruction errors with regard to the number of $\mathrm{q} 3$ modes retained. Case $\mathrm{R}$, to be compared to case $\mathrm{Q}$, again shows the weak dependency on the number of q4 modes.

The question now, in view of the discussion presented above, is how many timeframes are needed to generate an accurate first mode in axis four. To clarify this question, four new versions of tensor 1 have been generated using two equally spaced time-frames in axis 4 , four time-frames, eight time-frames and sixteen time-frames respectively. Then, HOSVD has been applied to these four tensors and the associated decompositions have been densified so as to reconstruct the original tensor retaining just one mode in axis 4 . The results obtained in terms of the average and maximum reconstruction errors, are presented in figure 4.9 where it could be observed that above six time-frames the errors remain practically constant. In fact, using merely four timeframes yields reconstruction errors only slightly larger than the limiting case of twenty time-frames. In practice, these results suggest that a less precise (and less expensive) camera could be used in this type of experiments because appropriate data reduction could replace the use of far too sophisticate equipment.

Regarding tensor 2, it could be observed in table 4.2 that retaining just one mode per axis allows for a rather accurate reconstruction of the information (the actual droplet breakup time). This reconstruction (line 1 of table 4.2) yields average and maximum errors of $0.7 \%$ and $1.8 \%$ respectively.

\subsubsection{Application Case 6}

The question to be addressed in this case is whether data belonging to all cases (with and without droplet breakup) can be integrated into a single tensor that can be meaningfully used for data analysis. To do it, some logical construction needs to be devised. The idea is that the vast majority of cases (though not all of them) that were not included in tensors 1 and 2 ended up with the droplet impinging on the airfoil before actual breakup took place. These cases involved either the lower airfoils velocities of $50 \mathrm{~m} / \mathrm{s}$ and $60 \mathrm{~m} / \mathrm{s}$, or the smallest model airfoil M3 $(\mathrm{Rc}=0.030 \mathrm{~m})$, or the smallest 


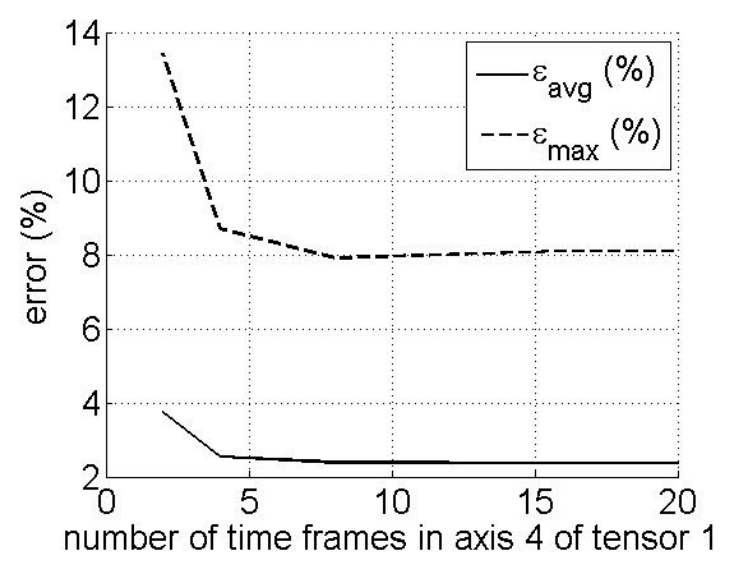

Figure 4.8: Mode 1 of axis 4 computed with 2, 4, 8 and 16 frames.

\begin{tabular}{|c|c|c|c|c|}
\hline \multicolumn{5}{|c|}{$\begin{array}{l}\text { Tensor } 2 \text {. Bins per axis } 3,2,4 \\
\text { Modes } \mathrm{q} 1, \mathrm{q} 2 \text {, and } \mathrm{q} 3 \text { retained per axis }\end{array}$} \\
\hline $\mathrm{q} 1$ & $\mathrm{q} 2$ & q3 & $\frac{1}{\epsilon_{a v}(\%)}$ & $\epsilon_{\max }(\%)$ \\
\hline 1 & 1 & 1 & 0,7 & 1,8 \\
\hline 2 & 2 & 2 & 0,3 & 1,0 \\
\hline 1 & 2 & 4 & 0,7 & 1,3 \\
\hline 3 & 1 & 2 & 0,7 & 1,3 \\
\hline 3 & 2 & 1 & 0,5 & 1,4 \\
\hline
\end{tabular}

Table 4.2: Errors associated to the reconstruction of tensor 2 when retaining different numbers of HOSVD modes per axis.

droplet of radius $(\mathrm{Rd}=200 \mu \mathrm{m})$. Breakup (with no impingement on the airfoil) was actually observed in a few cases, but its number was so small that it did not allow to fill in new data in tensors 1 and 2. Then, the idea is to extrapolate via HOSVD (as sketched in figure 4.9$)$ two velocity $(50 \mathrm{~m} / \mathrm{s}$ and $60 \mathrm{~m} / \mathrm{s})$, one leading edge radius $(0.030 \mathrm{~m})$ and one droplet radius $(200 \mu \mathrm{m})$ out of tensors 1 and 2 . The new tensors were called tensors 3 and 4 respectively. It should be stressed that the extrapolated data is virtual. That is: this new data predict what would happen in terms of the breakup process if instead of an approaching airfoil (bound for impingement) the droplet would sense the virtual continuation of the air velocity profile generated by the airfoil.

The way to ascertain whether the process thus described provides meaningful information is to select those few combinations of the newly added parameters in which breakup was present in the video recordings and compare this experimental data with the HOSVD reconstruction. An example of this comparison involving a limited number of cases is presented in table 4.3. In this table, the parameters that correspond to the new data of tensor 3 are shaded to facilitate identification.

The results presented in table 4.3 show that, in terms of the breakup time, the proposed approach yields average and maximum discrepancies of the order of $2 \%$ and $7 \%$ respectively. The process is, then, continued in the same way to generate the actual (virtual) time dependent deformation of the droplets. Four cases have been selected from table 4.3 and the comparison between the predicted and measured time dependent 
Tensors 3,4
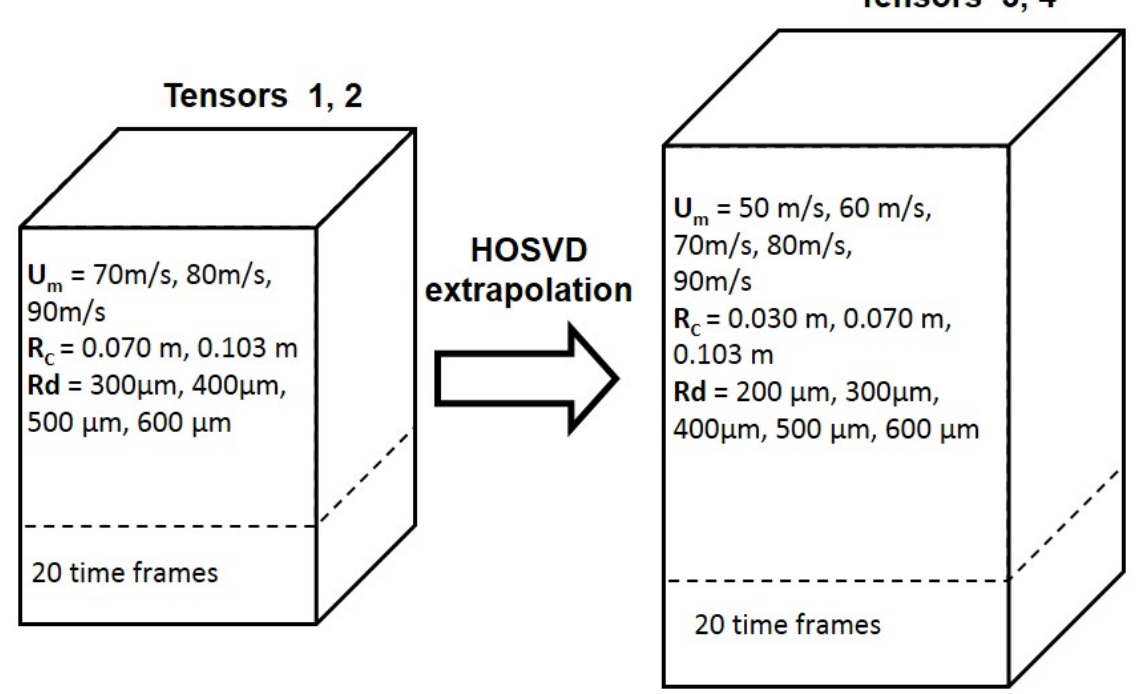

Figure 4.9: Sketch of the process followed in application case 6.

deformation is presented in figure 4.10 .

\subsubsection{Application Case 7}

In the previous application case, the tensor 2 (constructed from data of droplet that actually broke up) was used to extrapolate in the the first three axes: airfoil velocity (axis 1), airfoil leading edge radius (axis 2) and droplet radius (axis 3 ) to obtain the tensor 4. The question in this last case is whether an extrapolation in the time axis (axis 4) would exhibit a minimum in the minimum half-diameter for the breakup time. Note that this minimum indicates the onset of the breakup. In order to do this, both tensor 1 and tensor 2 are extrapolated in axis 4 to dimensionless time up to 1.05. Figure 4.11 show the comparison between the predicted and measured time dependent deformation for the three cases 1) $\mathrm{Um}=90 \mathrm{~m} / \mathrm{s} ; \mathrm{Rc}=0.103 \mathrm{~m} ; \mathrm{Rd}=400 \mu \mathrm{m}, 2) \mathrm{Um}=90 \mathrm{~m} / \mathrm{s} ; \mathrm{Rc}=0.70 \mathrm{~m}$; $\mathrm{Rd}=400 \mu \mathrm{m}$ and 3$) \mathrm{Um}=70 \mathrm{~m} / \mathrm{s} ; \mathrm{Rc}=0.103 \mathrm{~m} ; \mathrm{Rd}=400 \mu \mathrm{m}$. The average discrepancies for each case are $3.6 \%, 1.5 \%$ and $1.5 \%$ respectively, while the maximum discrepancies are $43 \%, 10 \%$ and $4 \%$. In any case, what is important here is that the minimum in the minimum half-diameter is indeed predicted. This suggest that all breakup information is stored in the deformation preceding the onset of the breakup.

\subsection{CONCLUSIONS}

A data reduction method based on HOSVD has been applied to a droplet deformation data set obtained in a rotating arm facility. The results show, that in the context of data reduction, HOSVD is a robust method that can be used with reasonable confidence to densify experimental data sets. In this way, the benefit (in terms of analysis) obtained from a limited set of experiments can be increased. However, this conclusion cannot 


\begin{tabular}{cccccc}
\hline $\mathrm{Um}(\mathrm{m} / \mathrm{s})$ & $\mathrm{Rc}(\mathrm{m})$ & $\mathrm{Rd}(\mu \mathrm{m})$ & $\operatorname{tr}(\mathrm{ms})$ & $\operatorname{Exp} \operatorname{tr}(\mathrm{ms})$ & HOSVD Discrepancy $(\%)$ \\
\hline 80 & 0,103 & $\mathbf{2 0 0}$ & 3,6 & 3,7 & 2,7 \\
90 & 0,103 & $\mathbf{2 0 0}$ & 3,0 & 3,2 & 7,1 \\
90 & 0,070 & $\mathbf{2 0 0}$ & 3,1 & 3,3 & 6,4 \\
$\mathbf{6 0}$ & 0,103 & 300 & 4,9 & 4,8 & 2,5 \\
$\mathbf{6 0}$ & 0,070 & 300 & 4,9 & 5,0 & 2,6 \\
90 & $\mathbf{0 , 0 3 0}$ & 300 & 3,3 & 3,3 & 0,0 \\
$\mathbf{6 0}$ & 0,103 & 400 & 4,8 & 4,7 & 1,3 \\
$\mathbf{6 0}$ & 0,070 & 400 & 4,9 & 4,9 & 1,0 \\
90 & $\mathbf{0 , 0 3 0}$ & 400 & 3,3 & 3,2 & 1,4 \\
$\mathbf{6 0}$ & 0,103 & 500 & 4,9 & 4,8 & 2,1 \\
$\mathbf{6 0}$ & 0,070 & 500 & 4,9 & 5,0 & 1,7 \\
90 & $\mathbf{0 , 0 3 0}$ & 500 & 3,3 & 3,2 & 0,8 \\
$\mathbf{6 0}$ & 0,103 & 600 & 4,8 & 4,7 & 2,0 \\
$\mathbf{6 0}$ & 0,070 & 600 & 4,9 & 5,0 & 1,3 \\
90 & $\mathbf{0 , 0 3 0}$ & 600 & 3,3 & 3,2 & 1,5 \\
\hline
\end{tabular}

Table 4.3: Comparisons between experimental data (Exp) and HOSVD reconstruction for some samples of tensor 3 .

be generalized in a broad sense because the robustness of the method depends on the actual problem under consideration. For example, HOSVD is not well suited to deal with situations that involve discontinuity type of flow behavior as it normally happens in experimental facilities such as shock tubes and supersonic wind tunnels. The reason is that, at the heart of the method, flow patterns are reconstructed by combining a limited set of HOSVD modes. Then, reconstruction of sharp discontinuities would require that all modes (considering them separately) contain accurate information on the discontinuity properties (position, for example) which is not normally the case, thereby yielding large reconstruction errors. Also, HOSVD is not an appropriate data reduction tool when some kind of chaotic flow pattern is involved.

As the first step, the experimental data set was divided into cases in which droplet breakup actually took place and cases in which it did not. Then the analysis was carried out on those cases where there was experimental evidence of breakup. HOSVD was used both to interpolate inside the data tensor and to extrapolate out of it. For the cases that have been considered, the average error associated to interpolation has been about $2 \%$, while the average error associated to extrapolations has been $5 \%$. In the case of the maximum errors, they were of the order of $20 \%$ for the interpolation cases, and of the order for $30 \%$ for the extrapolation cases. This suggests that, even though HOSVD should not be used to extrapolate outside the space of parameters where HOSVD modes were computed, the method can still provide meaningful results provided that the extrapolation is not too ambitious. In the case of interpolation, HOSVD proved to be a robust data reduction method capable of desifying datasets with reasonable accuracy.

As the second step, a virtual tensor was generated where estimated (virtual) breakup times (for cases where impingement on the incoming airfoil occurred prior to actual breakup) were computed extrapolating the HOSVD modes. Then, the process was repeated regarding the virtual time dependent droplet deformation. Then, the results obtained were compared with a limited number of experimental cases in which breakup did really occur. The average discrepancy between the experimental breakup time and the breakup time reconstructed via HOSVD was $5 \%$. In these cases the typical 


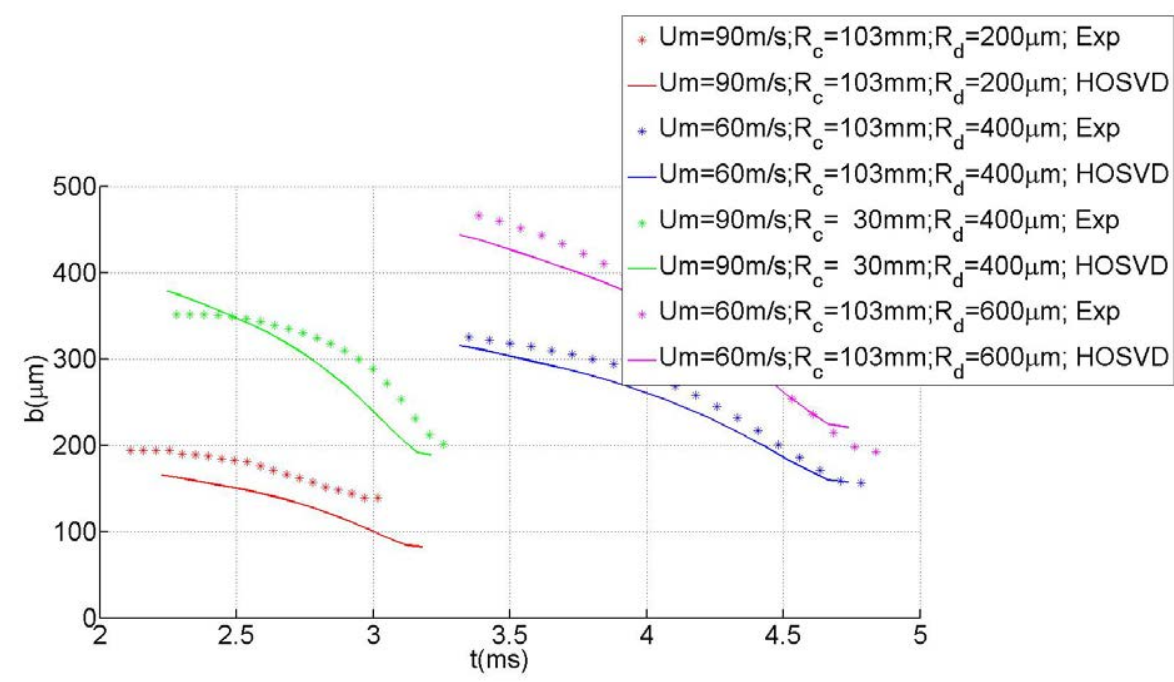

Figure 4.10: Comparison between measured and predicted time dependent droplet deformations for four cases selected from table 4.3.

average discrepancies in the time evolution of the droplet minimum half diameter were in the range from $5 \%$ to $10 \%$, while maximum discrepancies were of the order of $10 \%$ to $20 \%$.

It is also worth mentioning that even though twenty temporal frames were shot for each droplet trajectory, the analysis that has been performed suggests that accurate data can be reconstructed via HOSVD even if a much smaller number of frames, say four, are shot. This opens up the possibility of using less sophisticate (and less expensive) camera systems in this type of experiments.

Finally, the study that has been presented suggests that, when devising experimental campaigns that might be lengthy and/or costly, it might be of interest to plan for an approach that involves both the data reduction method (HOSVD in this case) and the experiments themselves co-operating in parallel along the campaign. In this sense, one of the advantages of HOSVD is that global modes can be computed from the outset even if the information is limited (albeit inaccurately). Then, as the amount of experimental information increases the modes evolve towards what would be their limiting state; thereby proving a natural quantitative criterion to stop the experiments because from some threshold onwards new information would be redundant. 


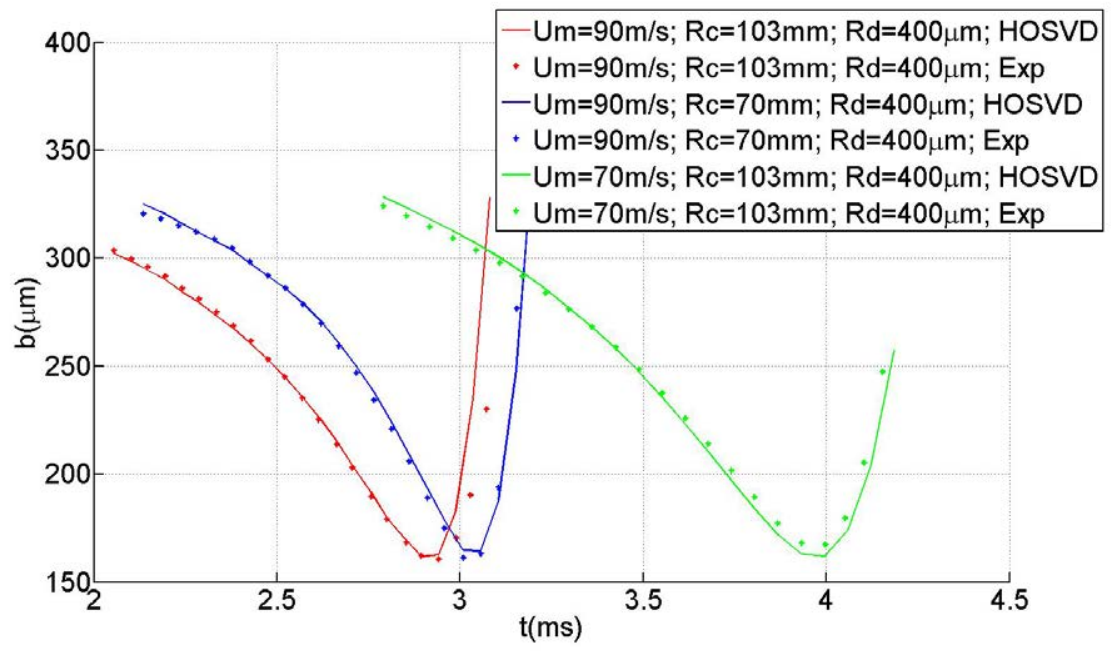

Figure 4.11: Comparison between measured and predicted time dependent droplet deformations for three cases after breakup has started. 


\section{Chapter 5}

\section{BREAKUP CRITERION}

A new breakup criterion is proposed in this chapter for droplets that suffer the flow field generated by an incoming airfoil. This criterion is based on the study of the characteristic times involved in the problem, which are the characteristic flow field variation time and the characteristic droplet deformation time. An empirical correlation is given to determine the Weber number at the onset of the breakup for each flow field and droplet size. Experimental data on the droplet deformation and breakup tests conducted in the rotating arm facility (the first and second experimental campaign) were used for the correlation. Droplets, in the range of 0.3 to $3.6 \mathrm{~mm}$ of diameter, were allowed to fall in the path of an incoming airfoil attached to the end of a rotating arm. Airfoil velocities varied between $50 \mathrm{~m} / \mathrm{s}$ and $90 \mathrm{~m} / \mathrm{s}$ while its leading edge radius ranged from 0.030 to $0.103 \mathrm{~m}$. The phenomenon was recorded by a high speed camera using shadowgraph illumination technique. The empirical correlation was obtained from droplets that breakup in the bag and stamen breakup mode. However the same correlation seems to be valid for droplets in the regime of shear breakup and for droplets in the regime of bag breakup.

\subsection{INTRODUCTION}

Traditionally, in the literature, two parameters are used mainly to determine the breakup of droplets that are suddenly exposed to an airstream: a critical Weber number or a maximum deformation attained. The Weber number is usually defined as

$$
W e=\left(\rho_{a} V_{s}^{2} D_{d}\right) / \sigma
$$

where $\rho_{a}$ is the air density, $\sigma$ is the surface tension, $D_{d}$ is the droplet diameter (equal to two times the droplet radius $R_{d}$ ) and $V_{s}$ is the slip velocity between the air velocity and the droplet velocity. The Weber number is the relation between the pressure forces, tending to deform the droplet and the surface tension forces that tend to keep the droplet within its initial shape. In shock tubes and wind tunnel facilities, the air velocity is assumed to be constant during the tests to determine this critical Weber number. Droplets suffer acceleration due to the airstream, however the time to breakup is so small that the droplet velocity is small compared to the air velocity when breakup takes place. This means that the Weber number based on the air velocity determines whether there exists breakup or not and the mode of the breakup. According 
to Guidelbencher [29] five modes of breakup can be distinguished: vibrational breakup (We $<12)$, bag breakup $(12<\mathrm{We}<50)$, bag and stamen breakup $(50<\mathrm{We}<100)$, shear breakup $(100<\mathrm{We}<350)$ and catastrophic breakup (We $>350)$. In the previous chapter 3 , it was observed the bag and stamen breakup for Weber number on the order of 38, which confirms the hypothesis that a new criteria should be given for the specific situation of the flow field generated by an incoming airfoil. Additionally, in particular, the Weber number evolved from 5 to 38 . That means that although the Weber was above 12 (the critical Weber number for bag breakup), no breakup was observed up to 38 because no enough time had passed when Weber attained the value of 12 . Therefore, there is an implicit coupling between how the velocity observed by the droplet increases and the ability of the droplet to deform and the critical Weber number at the breakup. Then it is necessary to define the breakup Weber as a function of the flow field variation and the droplet. This is in line with the suggestion in the conclusions of the chapter 3 of two parameters that should govern the deformation, one related to the flow field and the other one containing the droplet size and its properties. Regarding the maximum deformation attained, Taylor suggested that when the maximum diameter exceeds half the original diameter, breakup takes place [88]. This has been used in conjunction with deformation models such as TAB (Taylor Analogy Breakup Model) model [67] or DDB model [43] to determine the breakup. Although this has been proved to be a good approximation for sprays and for shock tubes experiments, for droplets those undergo a continuously increasing velocity, larger deformations have been observed.

\subsection{BREAKUP CRITERION PROPOSED: BAG AND STAMEN BREAKUP}

The vast majority of the video observed in the first campaign evidenced the same breakup mode, the so-called bag and stamen. An example of the video images recorded is shown in figure 5.1. As observed in the figure, the droplet undergoes a deformation period where the droplet can be approximated as an oblate spheroid whose maximum diameter increases as the airfoil approaches while the minimum diameter decreases. Then, at $\mathrm{T}=3.5 \mathrm{~ms}$, the minimum diameter starts to increase and the assumption of an oblate spheroid is no longer valid. Using Feo criteria [23], this is considered to be the onset of the breakup. In light of these results, it seems reasonable to use this criterion. The breakup process itself is better observed in figure 3.7. Once the breakup process has started, a hat-like shape is observed where the height of the hat is increasing until finally the tips of the hat forms a bag that explodes, while the center stamen still remains.

The deformation of these droplets was discussed and studied in the previous chapter 3 . The conclusions were that provided a parameter related to the flow field (acceleration times the square of the residence time) was constant and for the same diameter, the deformation rate was similar for the same slip velocity. Therefore, two parameters, one related to the flow field and another related to the droplet size should govern the process. Consequently, it seems plausible to think that both the flow field and the droplet size should govern also the breakup process. The droplet size could be important in the sense that the capacity that droplet has to absorb energy depends primary on the droplet size. In other words, the response time of the drop depends mainly on the droplet size. In order to address the breakup problem, it is interesting to study the characteristic times involved in the problem. First of all, a deformation characteristic time is defined as 


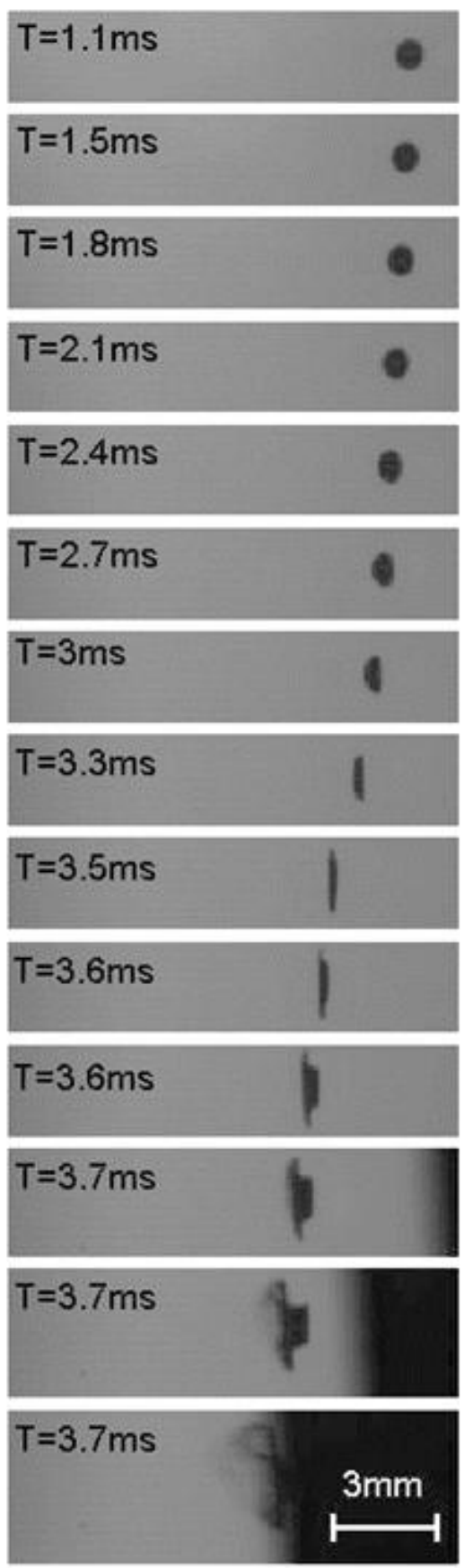

Figure 5.1: Example of the evolution of frames of the bag and stamen breakup mode of a droplet of $900 \mathrm{~m}$ of diameter, when M1 model was moving at $90 \mathrm{~m} / \mathrm{s}$. 


$$
t_{\text {def }}=\sqrt{\frac{\frac{4}{3} \pi R_{d}^{3} \rho_{w}}{\sigma}}
$$

where $\rho_{w}$ is the droplet density. The definition for this deformation time is connected to the Taylor Analogy Breakup model [67], where an analogy is established between a spring-mass system and a droplet being deformed. The surface tension force would be the spring tension force. For the variation of the flow velocity field, the characteristic time is defined as:

$$
t_{f f}=\frac{V_{s}}{d V_{s} / d t}
$$

The breakup instant should be related to these two characteristic times. The deformation and the breakup depend on the ability of the droplet to undergo deformation and the flow field force applied on the droplet. Therefore a new parameter that includes both effect, the $\tau$ parameter, is defined as the relation between these characteristic times:

$$
\tau=\frac{t_{d e f}}{t_{f f}}
$$

The breakup Weber number $W e_{B K}$, which is the value of the Weber number at the onset of the breakup, is known to define the breakup mode. The idea is to relate the breakup Weber number and the properties of the droplet and the flow field using the parameter $\tau$ defined. In figure 5.2, the breakup Weber number is plotted against the $\tau$ parameter for the total of 4902 droplets analyzed for bag and stamen breakup mode. It is observed a clear tendency in the data. As the $\tau$ parameter increases, the breakup Weber number increases. A linear approximation is depicted in the figure, being its equation:

$$
W e_{B K}=17,5268+17,8856 \tau
$$

This equation 5.4 is the breakup criterion proposed in this thesis. The parameter $\tau$ defined is specially meaning. On one hand, for a given flow field ( $t_{f f}$ constant), if the droplet response time $t_{d e f}$ increases, which means that it takes more time to the droplet to notice the flow field applied to it, a higher Weber number can be attained. On the other hand, for a given droplet ( $t_{d e f}$ constant), if the characteristic time for the variation of the flow velocity field $t_{f f}$ decreases, the Weber number attained increases.

In the chapter 3 , a characterization of the flow field without the presence of the drop was made and near the model, where the droplet usually breakup, an approximation for the air velocity $u$ was made as a function of the distance to the model $x$.

$$
u=U_{m}-8,28 U_{m} \frac{x}{c}
$$

Taking into account equation 5.5 and assuming that the droplet velocity at the breakup is much smaller that the air velocity and neglecting it in the slip velocity, the characteristic time for the variation of the flow field velocity can be approximated as:

$$
t_{f f}=\frac{V_{s}}{d V_{s} / d t} \simeq \frac{1}{8,28} \frac{c}{U_{m}}
$$




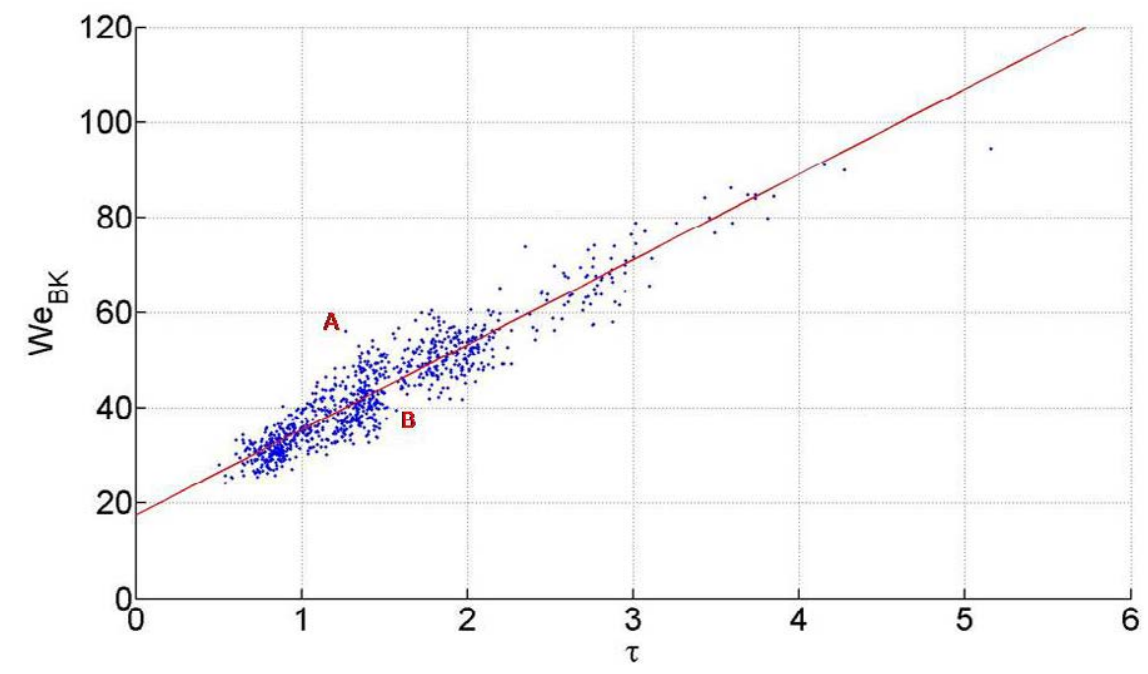

Figure 5.2: Breakup criterion applied to droplets that breakup in the mode of bag and stamen breakup.

It is interesting to simplify the $\tau$ parameter using this simplification. Then the dependency of the $\tau$ parameter on the model velocity, model chord and droplet radius can be established:

$$
\tau \propto \frac{U_{m}}{c} R_{d}^{3 / 2}
$$

This means that if increasing breakup Weber number were desired to obtain for example other regimes of breakup, then either the droplet radius should be increased or the chord length should decrease. The dependency on the droplet radius is stronger (one and half power), than the model velocity or the model chord.

It is also observed in figure 5.2 that the breakup criterion provides the breakup Weber number with a spread on about \pm 10 . In order to explain this spread, attention should be paid on the way to define the onset of the breakup. Figures 5.3 and 5.4 show the evolution of frames recorded and the value of $\tau$ and We for each frame near the breakup for two droplets, droplet A and B depicted in figure 5.2, one above the spread and the other below. Droplet A corresponds to a droplet of $900 \mathrm{~m}$ of diameter, $90 \mathrm{~m} / \mathrm{s} \mathrm{of}$ model velocity and M1 model. Droplet B corresponds to a droplet of $680 \mathrm{~m}$ of diameter, $80 \mathrm{~m} / \mathrm{s}$ of model velocity and M2 model. It is shown in bold the breakup instant and T stands for the time.

Figure 5.3 and 5.4 show the difficulty to determine the minimum in the minimum diameter, which has been considered as the onset of the breakup, since little variation is observed between consecutive frames. This is also confirmed in the plots of figure 5.5, which show the evolution of minimum diameter for droplets A and B. Each marker corresponds to a frame and the curve fit for the minimum diameter was calculated automatically as a polynomial of order 12. The onset of the breakup is then calculated as the global minimum of this curve. It can be observed in figure 5.5 that the minimum in the curve comprises around 8 frames. On the other hand, a variation of 8 frames is translated into a variation of 10 in the Weber number (see figures 5.3 and 5.4), which 


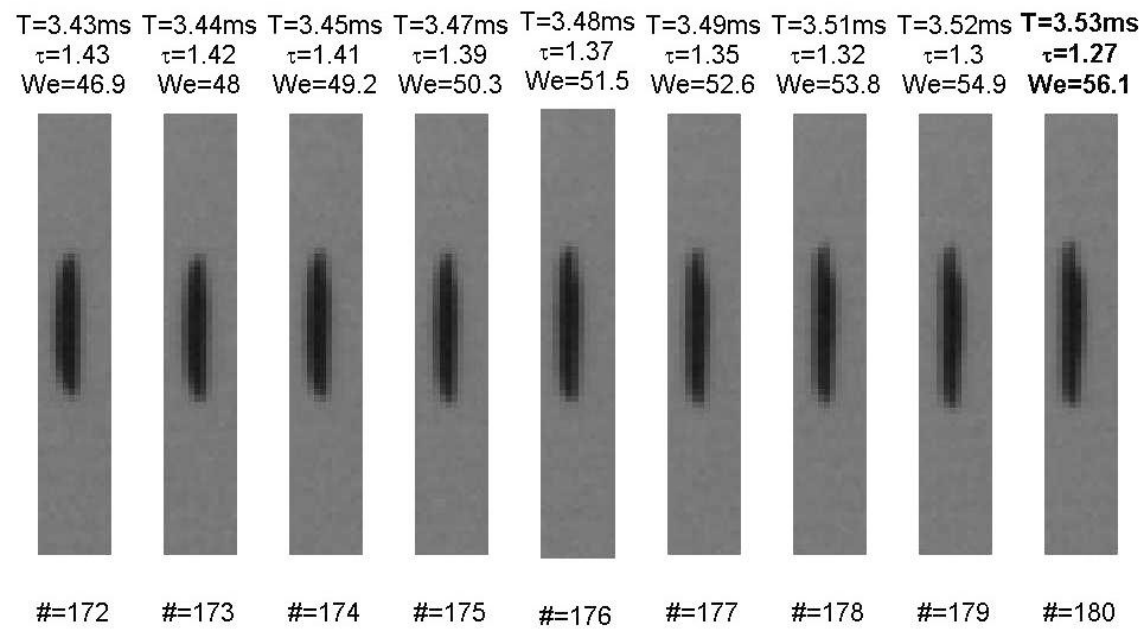

Figure 5.3: Evolution of frames near the breakup for droplet A.

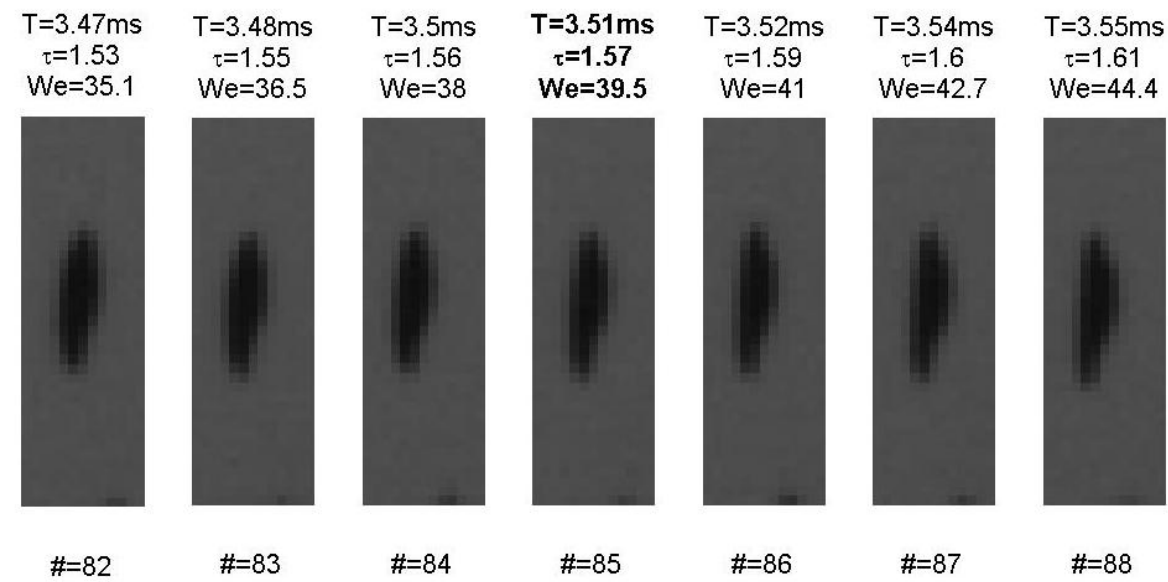

Figure 5.4: Evolution of frames near the breakup for droplet B.
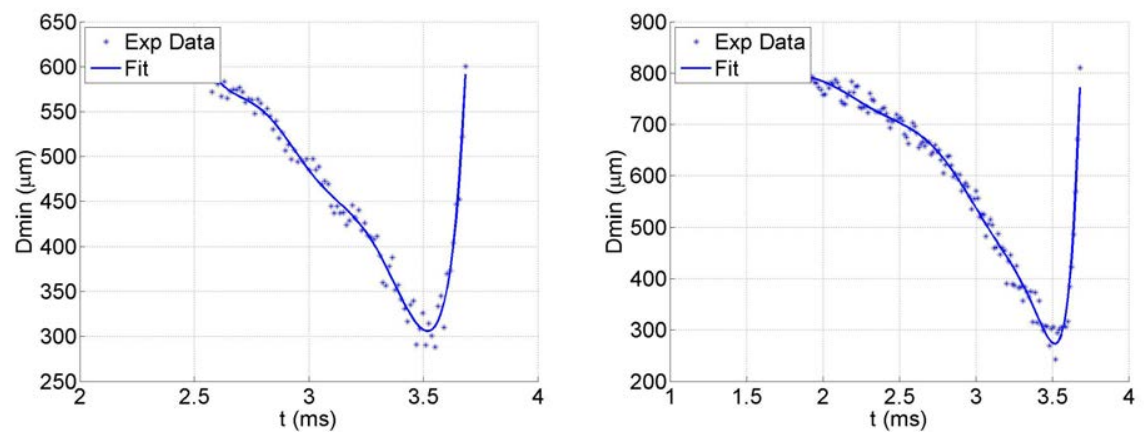

Figure 5.5: Evolution of minimum diameter for droplet A (left) and B (right). 
explain the spread founded in figure 5.1.

\subsection{BAG BREAKUP}

Although the vast majority of the breakup cases found during the first experimental campaign was the type bag and stamen mode, a few limited cases of bag breakup were also found. The sequence of images taken for one of these cases is found in figure 5.6.

It is observed in figure 5.6 that there exist a similar period where droplet deforms as an oblate spheroid. Then a cavity is formed and the spheroid is curved until finally the bag is observed. The difficulty here to define the onset of the breakup is the curvature observed prior to form the bag. However and for sake of simplicity, the same criterion is used and the onset of the breakup is defined as the minimum in the minimum diameter of the superimposed ellipsoid. Following this criterion, the few cases of bag breakup found were added to the plot of figure 5.2 and are shown in figure 5.7. It is observed that these new cases fit well with the fitted curve for the cases of bag and stamen.

The spread found can be explained by the difficulty found in defining the onset of the breakup when compared to bag and stamen. The evolution of frames near the breakup is shown in figure 5.8 , which confirm this hypothesis.

\subsection{SHEAR BREAKUP}

During the second experimental campaign a different breakup mode was found for some droplets, the so-called shear/sheet stripping breakup. A total of 25 droplets were selected, analyzed and studied. Table 5.1 shows the 25 five cases selected:

An example (case shear \#1) that evidenced this mode of breakup is shown in figure 5.9 , where a droplet of $3.2 \mathrm{~mm}$ of diameter was exposed to the flow field generated by M1 model (chord $0.690 \mathrm{~m}$ ) moving at $90 \mathrm{~m} / \mathrm{s}$.

In figure 5.9, it is shown a clear typical shear/sheet stripping breakup mode. Initially, the droplet undergoes a similar deformation (oblate spheroid) as in bag and stamen breakup. However, this deformation period last less than before ( $\mathrm{T}=2 \mathrm{~ms}$ ), and it appear a small ring wave in the front $(\mathrm{T}=2.8 \mathrm{~ms})$, that goes downstream until it finally explodes $(\mathrm{T}=2.9 \mathrm{~ms})$.

Following with the same line of thinking on defining the onset of the breakup as the instant when the minimum diameter starts to increase, for five meaningfully cases including the case shown in figure 5.9, the droplet frames evolution near the minimum in the minimum diameter are shown in figure 5.10. It can be observed in figure 5.10 that, although all of them seem to be shear breakup when the video is watched, the actual shape is different as the Weber number increases (due to the increase in droplet diameter form $0.96 \mathrm{~mm}$ to $3.2 \mathrm{~mm}$ ). Starting from the bottom, the deformation process in case Shear \#18 (Dd= $0.960 \mathrm{~mm})$ is very similar to that of bag and stamen breakup and the shape is similar to an oblate spheroid, so the same criteria can easily be applied. As the Weber number increases, it can be observed that more difficulty is encountered to define the onset of the breakup. For case Shear \#3 (Dd= $1.527 \mathrm{~mm})$, it seems like an inverted hat shape (the air is coming from the right), where the front wave seems more spherical. Then, for case Shear \#1 (Dd= $2.628 \mathrm{~mm})$, the front wave shows also a prominence, while finally in case Shear \#7 ( $\mathrm{Dd}=3.2 \mathrm{~mm})$, it seems to appear the initiation of a second wave in this prominence. The superimposed ellipsoid is not able to be used for these last cases; therefore a new criterion is adopted. The criterion chosen 


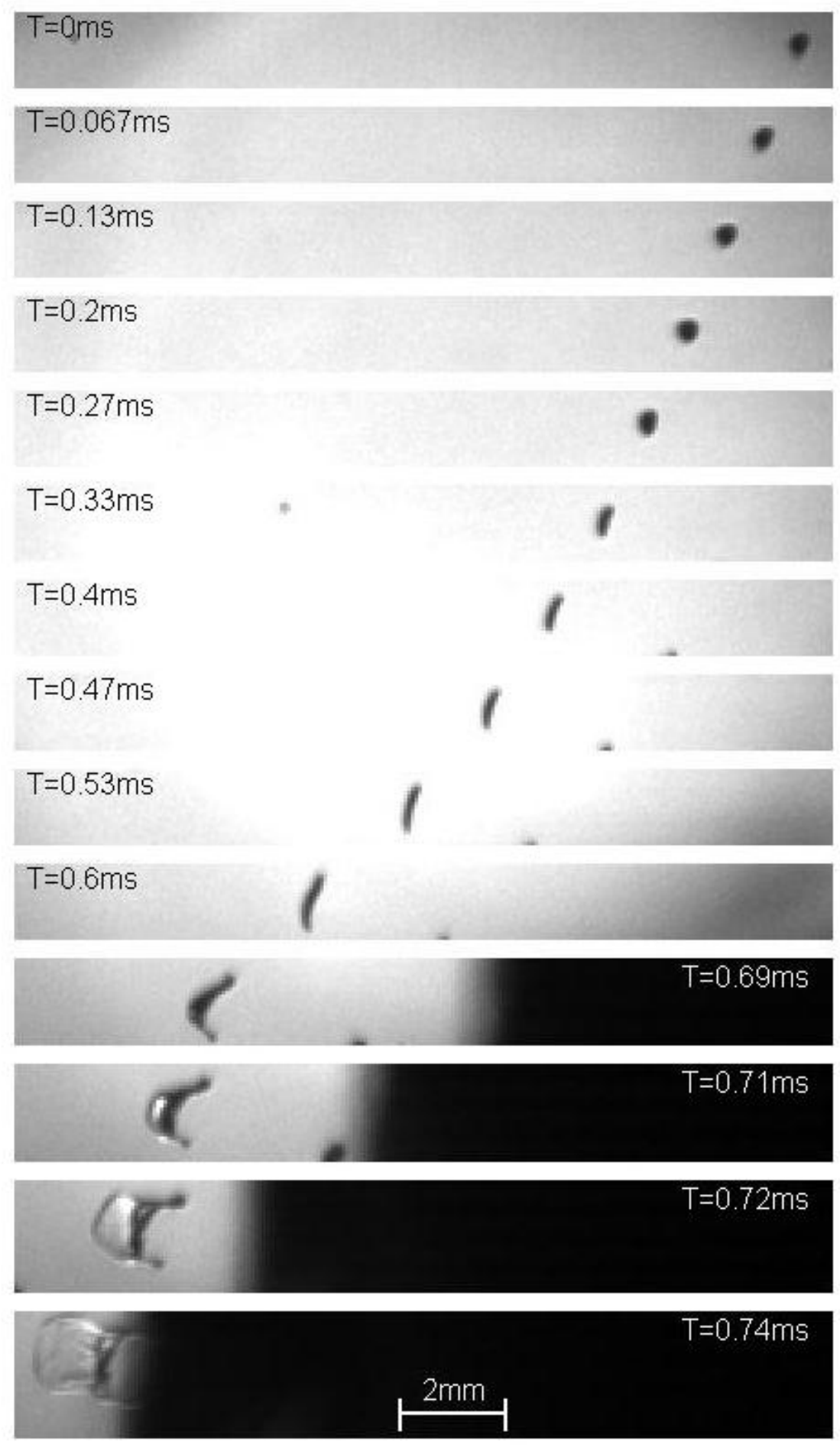

Figure 5.6: Example of the evolution of frames of the bag breakup mode of a droplet of $191 \mathrm{~m}$ of diameter, when M1 model was moving at $90 \mathrm{~m} / \mathrm{s}$ (Bag \#1). 


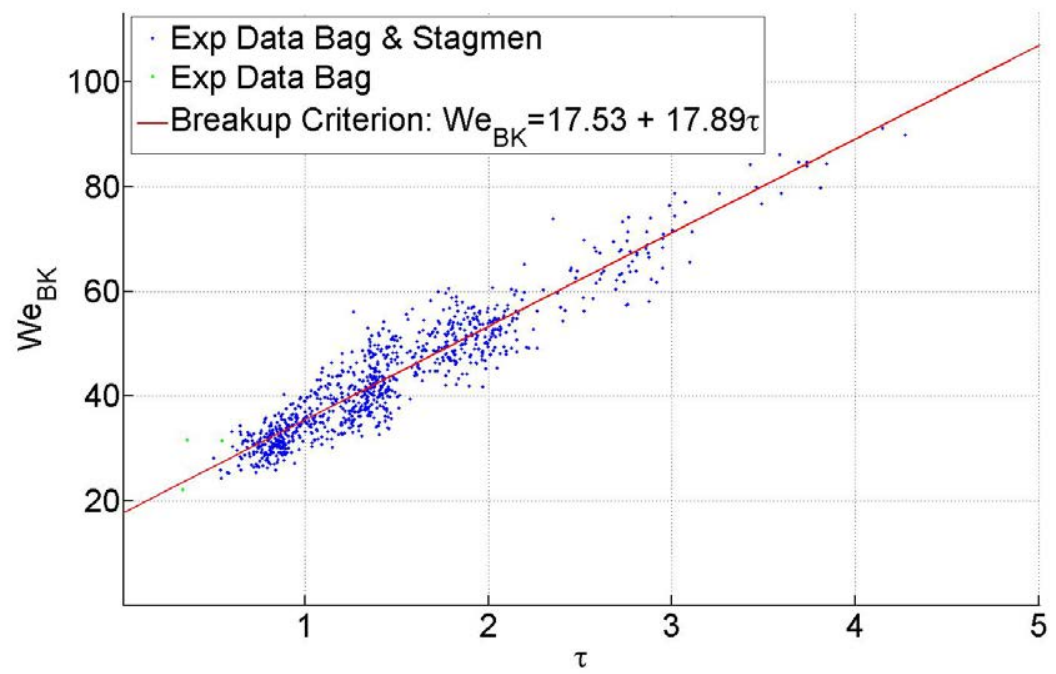

Figure 5.7: Breakup criterion applied to droplets that breakup in the modes of bag and bag and stamen breakup.

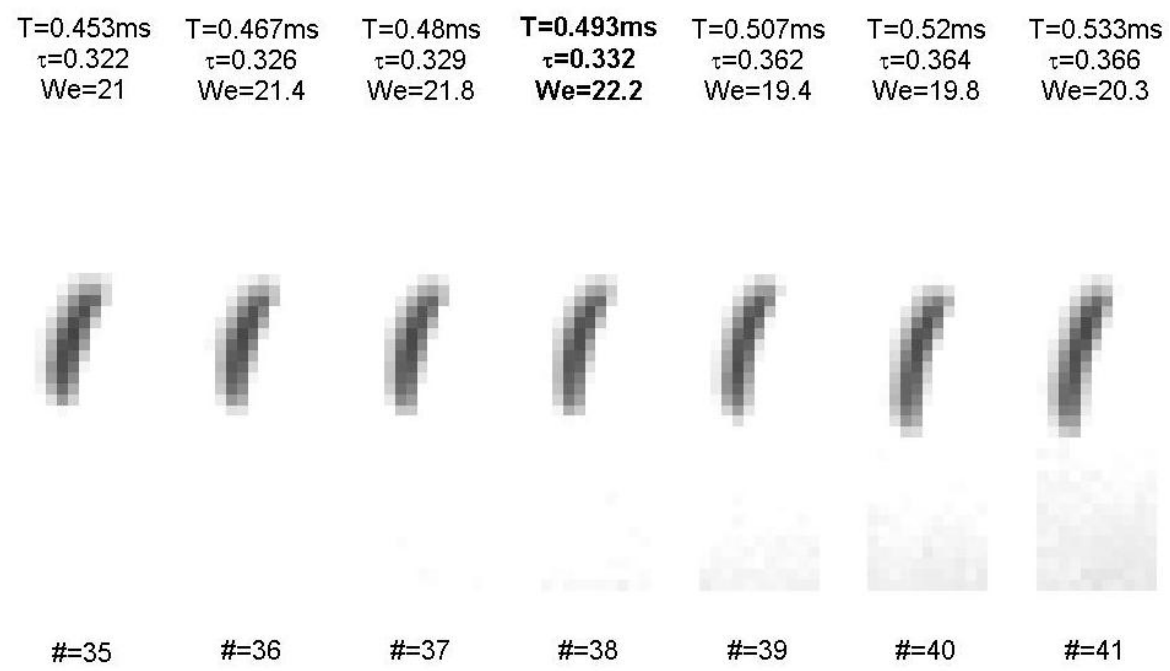

Figure 5.8: Evolution of frames near the breakup for the same droplet of figure 14 (Bag \#1). 


\begin{tabular}{cccc}
\hline Case & Um $(\mathrm{m} / \mathrm{s})$ & $\mathrm{cm}(\mathrm{mm})$ & Dd $(\mathrm{tgm})$ \\
\hline Shear \#1 & 90 & 690 & 3201 \\
Shear \#2 & 90 & 690 & 3103 \\
Shear \#3 & 90 & 468 & 1527 \\
Shear \#4 & 90 & 468 & 1444 \\
Shear \#5 & 90 & 468 & 1702 \\
Shear \#6 & 90 & 468 & 1640 \\
Shear \#7 & 90 & 468 & 2628 \\
Shear \#8 & 90 & 468 & 2957 \\
Shear \#9 & 90 & 468 & 2948 \\
Shear \#10 & 80 & 468 & 2489 \\
Shear \#11 & 80 & 468 & 2344 \\
Shear \#12 & 80 & 468 & 2804 \\
Shear \#13 & 80 & 468 & 2460 \\
Shear \#14 & 90 & 468 & 1006 \\
Shear \#15 & 90 & 468 & 973 \\
Shear \#16 & 90 & 468 & 976 \\
Shear \#17 & 90 & 468 & 1050 \\
Shear \#18 & 90 & 468 & 960 \\
Shear \#19 & 90 & 468 & 1038 \\
Shear \#20 & 90 & 468 & 1019 \\
Shear \#21 & 90 & 468 & 989 \\
Shear \#22 & 90 & 468 & 1044 \\
Shear \#23 & 90 & 468 & 994 \\
Shear \#24 & 90 & 468 & 1009 \\
Shear \#25 & 90 & 468 & 999 \\
\hline
\end{tabular}

Table 5.1: Comparisons between experimental data (Exp) and HOSVD reconstruction for some samples of tensor 3 . 

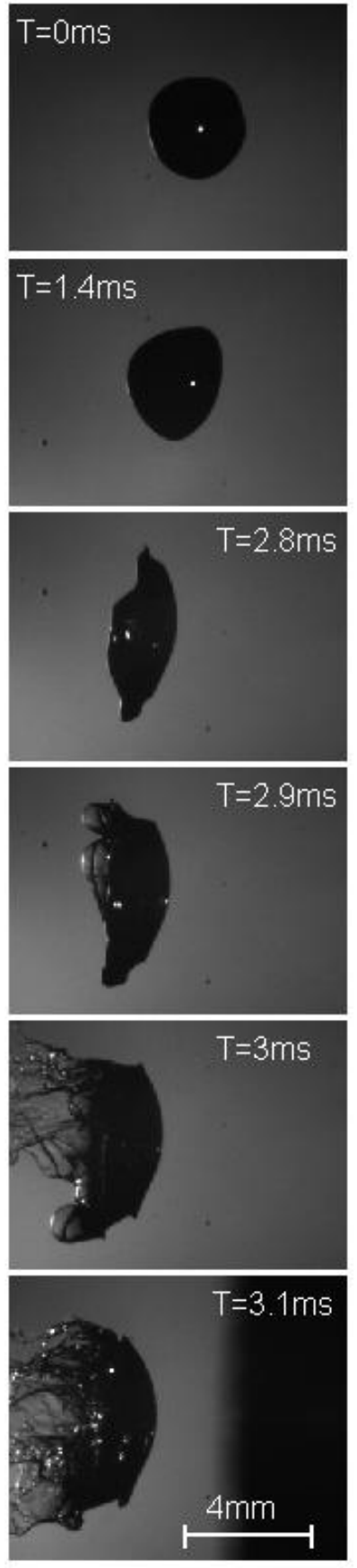

Figure 5.9: Example of the evolution of frames of the shear breakup mode of a droplet of $3.2 \mathrm{~mm}$ of diameter, when M1 model was moving at $90 \mathrm{~m} / \mathrm{s}$ (case Shear \#1). 


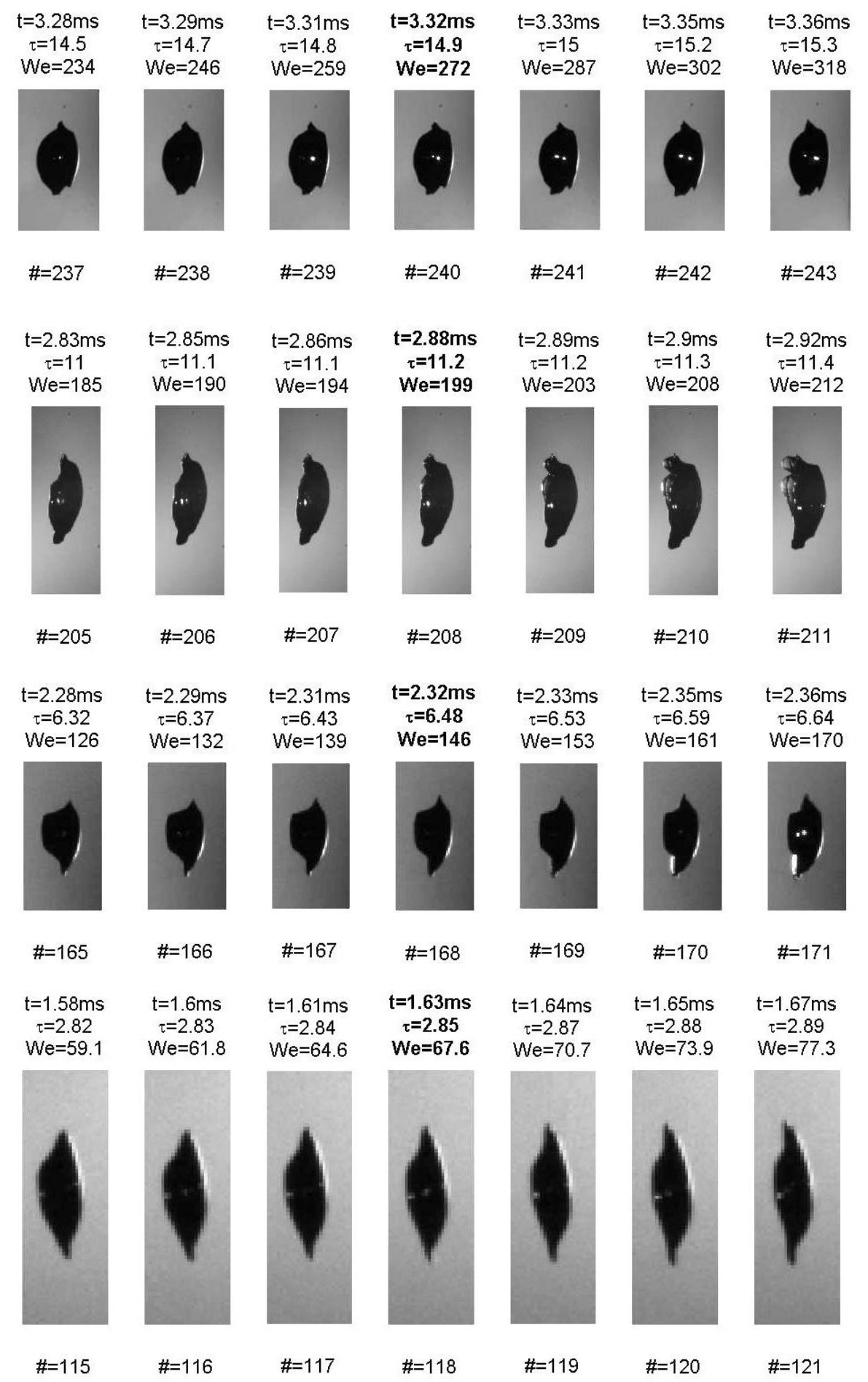

Figure 5.10: Evolution of frames near the minimum in the minimum diameter for cases Shear \#7, Shear \#1, Shear \#3 and Shear \#18 from the top to the bottom. 


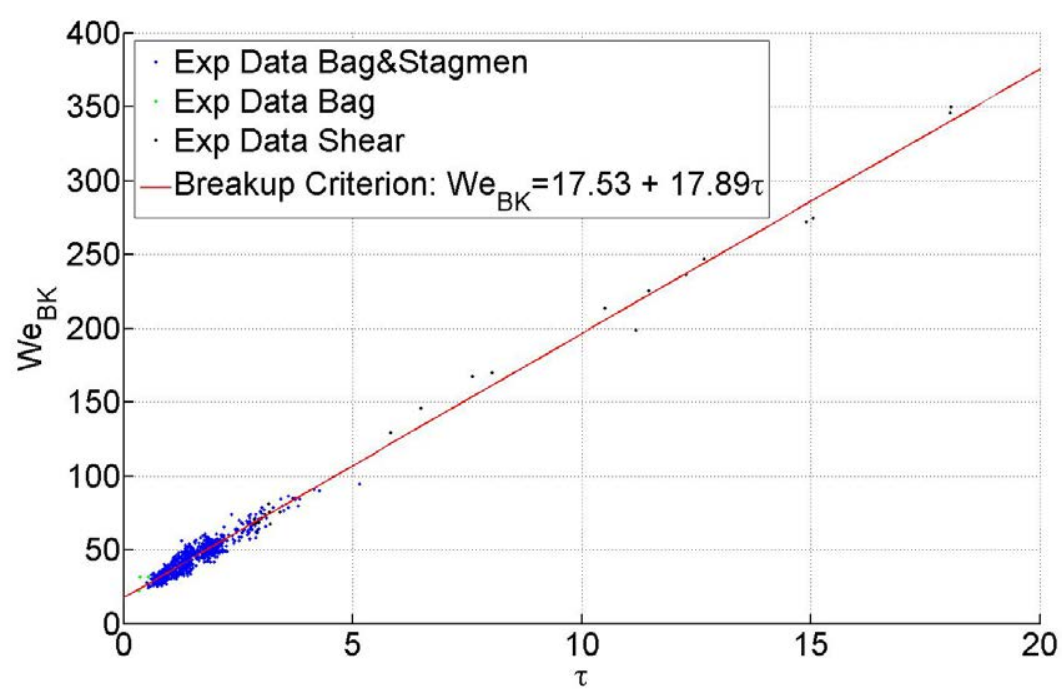

Figure 5.11: Breakup criterion

is the moment at which the front wave passes the middle of the droplet. In figure 5.10, it is shown in bold the breakup instant chosen for each case.

Finally, and using the criterion previous discussed, the breakup points corresponding to the few cases of bag breakup mode and the 25 cases of shear breakup were added to plot from which the breakup criterion was obtained (see figure 5.11). It can be observed in figure 5.11, that breakup points for the shear breakup mode also fit in the same line corresponding to the breakup criterion for bag and stamen and bigger Weber number are obtained for this new mode of breakup. The few cases of bag breakup correspond to the low limit of the cases of bag and stamen, while shear breakup cases correspond to the upper limit.

\subsection{CONCLUSIONS}

Droplet breakup regimes modes were investigated experimentally for droplets in the vicinity of an incoming airfoil. Three regimes modes namely bag, bag and stamen and shear breakup were founded although the vast majority of the cases were bag and stamen type. Feo criteria [23] (minimum in the minimum diameter of the superimposed ellipsoid) was used for bag and bag and stamen breakup. For shear breakup, a new definition for the onset of the breakup process was proposed, being this moment defined as the instant when the forward wave passed the middle of the droplet. Following these definitions, a unique criterion is proposed for the breakup:

$$
W e_{B K}=17,5268+17,8856 \tau
$$

The $\tau$ parameter is the relation between a characteristic deformation time and a velocity variation characteristic time, and contains the influence in the breakup of the ability of the droplet to deform and the flow field variation. This leads to the conclusion that both the droplet ability to deform (determined by the droplet size) and the flow field variation govern the process leading to the breakup. 
The criterion proposed worked well for the three breakup regimes within the range of parameters analyzed (droplets diameter from $150 \mu \mathrm{m}$ to $3200 \mu \mathrm{m}$, model leading radius from 0.029 to $0.103 \mathrm{~m}$, and model velocities from 50 to $90 \mathrm{~m} / \mathrm{s}$ ). This criterion can be applied in ice accretion codes for aircraft icing. The spread in the data found was explained by the difficulty in the definition of the breakup. 


\section{Chapter 6}

\section{CONCLUSIONS}

The problem of droplet breakup in the vicinity of the leading edge of an airfoil has been addressed. An experimental investigation has been conducted in the rotating arm facility at INTA covering droplets diameter from $0.3 \mathrm{~mm}$ to $3.6 \mathrm{~mm}$. Droplets were generated and allow to fall in the path of an incoming airfoil. Three airfoil sizes (leading edge radius of $0.103 \mathrm{~mm}, 0.070 \mathrm{~mm}$, and $0.029 \mathrm{~mm}$ ) and five airfoil velocities $(50 \mathrm{~m} / \mathrm{s}, 60 \mathrm{~m} / \mathrm{s}, 70 \mathrm{~m} / \mathrm{s}, 80 \mathrm{~m} / \mathrm{s}$ and $90 \mathrm{~m} / \mathrm{s}$ ) were used during the test. By means of shadowgraph technique, video images of the deformation and the breakup of the droplets were recorded. The flow field generated by these airfoils was characterized in advance using PIV technique. A tracking software was developed to obtain quantitative data on the droplet deformation and the breakup from the images.

First, a characterization of the specific flow field that droplets actually senses when an airfoil is approaching is made and the principal flow parameters involved in the problem of deformation and breakup of droplets are obtained. Then, a data reduction method, the so-called HOSVD, was applied to the problem aiming to provide insight in the underlying physics. Two tensor were constructed: one containing the deformation information and the other containing the breakup time. These tensor were used to extrapolate outside the tensor and to interpolate inside. And finally, the definitions of the onset of the breakup for each mode have been discussed and a breakup criterion equation has been proposed. The main conclusions can be stated as follows:

- In the most of the cases that were addressed during the experimental campaigns, the breakup type was 'bag and stamen'. In a few cases 'bag' and 'shear' breakup were also identified.

- Unsteady effects due to unsteady slip velocity and acceleration profiles play a critical role in the droplet deformation and breakup processes. In the cases addressed in this thesis (continuously accelerating flow) the effect was to anticipate significantly the onset of breakup.

- It was found that if the flow acceleration profile times the square of the droplet residence time is constant, droplet deformation (its instant aspect ratio) depends on the slip velocity only. this suggests that the problem is, at least, governed by a parameter that involves two characteristic times: the characteristic time of the flowfield variation and the droplet residence time.

- This thesis is the first step, only, in a long term development process aiming to generate reliable engineering methods to predict droplet behavior in the vicinity 
of aircraft wings. This requires the availability of reliable databases that can be used for algorithm development purposes. Because of their own nature, the experiments involved are complex and expensive. Then, in this context, it has been found that High Order Singular Value Decomposition is a rather adequate data reduction method for this problem. The reason is that it allows for the generation of clean and densified databases (that are obtained after a limited set of experiments) that can readily be used for model development purposes.

- A new breakup criterion has been proposed to predict breakup in the 'bag and stamen' mode that has prove to be prevailing one in the majority of experimental cases that were addressed in this thesis. Again, the breakup criterion depends on a dimensionless time that is the ratio of the characteristic droplet deformation time to the flow field typical variation time. 


\section{Bibliography}

[1] R. J. Adrian. Particle-imaging techniques for experimental fluid mechanics. Annual review of fluid mechanics, 23(1):261-304, 1991.

[2] S. Alesekyenko, M. Sinapius, M. Schulz, and O. Prykhodko. Interaction of supercooled large droplet with aerodynamic profile. In SAE Technical paper 2015-01-2118, 2015. doi:10.4271/2015-01-2118.

[3] C. N. Aliaga, M. S. AubÃl', G. S. Baruzzi, and W. G. Baruzzi. FENSAP-ICEunsteady: Unified in-flight icing simulation methodology for aircraft, rotorcraft, and jet engines. Journal of Aircraft, 48:119-126, 2011.

[4] D. Althaus. Niedrig-geschwindigkeits-profile. In Vieweg and Sohn Verlagsgesell-schaft, page 555. 1996.

[5] J. Alzaili and D. Hammond. Experimental investigation of thin water film stability and its characteristics in SLD icing problem. SAE International, 2011. 2011-38-0064.

[6] D. N. Anderson and A. Feo. Ice-accretion scaling using water-film thickness parameters. 2002.

[7] R. Benay. A global method of data reduction applied to seven-hole probes. Experiments in Fluids, 54(6):1-16, 2013.

[8] R. B. Bird, W. E. Stewart, and E. N. Lightfoot. Transport Phenomena. New York, 2002.

[9] M. B. Bragg. Aerodynamics of supercooled-large-droplet ice accretion and the effect on aircraft control. In Proceedings of the FAA International Conference on Aircraft Inflight Icing, volume 2, pages 387-399, 1996.

[10] M. B. Bragg and E. Loth. Effects of large-droplet ice accretion on airfoil and wing aerodynamics and control. Technical report, NASA, 2000.

[11] F. Caliskan and C. Hajiyev. A review of in-flight detection and identification of aircraft icing and reconfigurable control. Progress in Aerospace Sciences, 60:12 $-34,2013$.

[12] S. E. Campbell, A. P. Broeren, and M. B. Bragg. Sensitivity of aircraft performance to icing parameter variations. Journal of Aircraft, 44(5):1758 - 1760, 2007. 
[13] S. Chang, C. Wang, and M. Leng. Modeling of droplet deformation and breakup in the supercooled large droplet regime. In SAE Technical paper 2015-01-2119, 2015. doi:10.4271/2015-01-2119.

[14] H. Chen. Two-dimensional simulation of stripping breakup of a water droplet. AIAA Journal, 46(5):1135-1143, May 2008.

[15] R. Clift, J. R Grace, and M. E Weber. Bubbles, drops, and particles. Courier Corporation, 2005.

[16] S. G. Cober, G. Isaac, A. D. Shah, and R. Jeck. Defining characteristic cloud droplet spectra from in-situ measurements. In AIAA 41 st Aerospace Sci. Meeting and Exhibit, 2003.

[17] G. E. Cossali. A fourier transform based data reduction method for the evaluation of the local convective heat transfer coefficient. International journal of heat and mass transfer, 47(1):21-30, 2004.

[18] L. De Lathauwer, B. De Moor, and J. Vandewalle. A multilinear singular value decomposition. SIAM Journal on Matrix Analysis and Applications, 21:1253$1342,2000$.

[19] L. De Lathauwer, B. De Moor, and J. Vandewalle. On the best rank-1 and rank( $\mathrm{r} 1, \mathrm{r} 2, \ldots, \mathrm{rn})$ approximation of higher-order tensors. SIAM Journal on Matrix Analysis and Applications, 21(4):1324-1342, 2000.

[20] Y. Dong and J. Ai. Research on inflight parameter identification and icing location detection of the aircraft. Aerospace Science and Technology, 29(1):305$312,2013$.

[21] P. Druault, P. Guibert, and F. Alizon. Use of proper orthogonal decomposition for time interpolation from piv data. Experiments in fluids, 39(6):1009-1023, 2005.

[22] E. C. English and F. R. Fricke. The interference index and its prediction using a neural network analysis of wind-tunnel data. Journal of Wind Engineering and Industrial Aerodynamics, 83(1):567-575, 1999.

[23] A. Feo, M. Vargas, and S. Sor. Rotating rig development for droplet deformation/breakup and impact induced by aerodynamic surfaces. SAE International 2011-38-0087, 2011.

[24] D. W. Hammond G. Luxford and P. Ivey. Role of droplet distortion and break-up in large droplet aircraft icing. AIAA paper 2004-411, 2004.

[25] A. García-Magarino, S. Sor, and A. Velazquez. Experimental characterization of water droplet deformation and breakup in the vicinity of a moving airfoil. Aerospace Science and Technology, 45:190-500, 2015.

[26] A. García-Magarino, S. Sor, and A. Velazquez. Data reduction method for droplet deformation experiments based on high order singular value decomposition. Experimental Thermal and Fluid Science, 79:13 - 24, 2016.

[27] R. W. Gent, N. P. Dart, and J. T. Cansdale. Aircraft icing. The royal society, 358:2873-2911, 2000. 
[28] C. Ghenai and C. X. Lin. Verification and validation of nasa lewice 2.2 icing software code. Journal of Aircraft, 43(5):1253 - 1258, 2006.

[29] D. R. Guildenbecher, C. Lopez-Rivera, and P. E. Sojka. Secondary atomization. Experiments in Fluids, 46(3):371-402, 2009.

[30] J. Han and G. Trygggvason. Secondary of axissymmetric liquid drops. I. Acceleration by a constant body force. Physics of fluids, 11(12):3650-3667, December 1999.

[31] J. Han and G. Trygggvason. Secondary of axissymmetric liquid drops. II. Impulsive acceleration. Physics of fluids, 13(6):1554-1565, June 2001.

[32] Y. Han and J. Palacios. Airfoil-performance-degration prediction based on nondimensional icing parameters. AIAA Journal, 51(11):2570-2581, November 2013. DOI: $10.2514 /$ I.J052207.

[33] R. J. Hansman. The effect of the atmospheric droplet size distribution on aircraft ice accretion. In AIAA 22nd Aerospace sciences meeting, Reno, Nevada, january 1984.

[34] R. J. Hansman. The influence of ice accretion physics on the forecasting of aircraft icing conditions. Technical Report 19900011612, NASA, March 1990. 90N20928.

[35] K. Hasanzadeh, E. Laurendeau, , and I. Paraschivoiu. Quasi-steady convergence of multistep navierâĂŞstokes icing simulations. Journal of Aircraft, 50(4):1261-1274, 2013.

[36] J. O. Hinze. Fundamentals of the hydrodynamic mechanism of splitting in dispersion processes. A. I. Ch. E. Journal, 1(5):289-295, September 1955.

[37] F. Homann. Der einfluss grosser zähigkeit bei der strömung um den zylinder. ZAMM-Journal of Applied Mathematics and Mechanics/Zeitschrift für Angewandte Mathematik und Mechanik, 16(1036):153-164, 2015.

[38] R. Honsek, W. G. Habashi, and M. S. Aubé. Eulerian modeling of in-flight icing due to supercooled large droplets. Journal of Aircraft, 45(4):1290-1296, July-August 2008. DOI: 10.2514/1.34541.

[39] J. Hospers and H. Hoeijmakers. Numerical simulation of SLD ice accretions. SAE Technical Paper 2011-38-0071, 2011. doi:10.4271/2011-38-0071.

[40] L. P. Hsiang and G. M. Faeth. Near-limit drop deformation and secondary breakup. International Journal of Multiphase Flow, 18(5):635-652, 1992.

[41] L. P. Hsiang and G. M. Faeth. Drop properties after secondary breakup. International Journal Multiphase Flow, 19(5):721-735, 1993.

[42] J.-S. Hwang, A. Kareem, and H. Kim. Wind load identification using wind tunnel test data by inverse analysis. Journal of Wind Engineering and Industrial Aerodynamics, 99(1):18-26, 2011.

[43] E. A. Ibrahim, H. Q. Yang, and A. J. Przekwas. Modeling of spray droplets deformation and breakup. Journal of Propulsion and Power, 9(4):651-654, 1993. 
[44] O. Igra and K. Takayama. Shock tube study of the drag coefficient of a sphere in a non-stationary flow. Proceedings of the Royal Society of London. Series A: Mathematical and Physical Sciences, 442(1915):231-247, 1993.

[45] E. Iuliano, G. Mingione, F. Petrosino, and F. Hervy. Eulerian modeling of large droplet physics towards realistic aircraft icing simulation. Journal of Aircraft, 48(5):1621-1632, September-October 2011. DOI: 10.2514/1.C031326.

[46] G. Jourdan, L. Houas, O. Igra, J.-L. Estivalezes, C. Devals, and E. E. Meshkov. Drag coefficient of a sphere in a non-stationary flow: new results. Proceedings of the Royal Society A: Mathematical, Physical and Engineering Science, 463(2088):3323-3345, 2007.

[47] S. K. Jung and R. S. Myong. A second-order positivity-preserving finite volume upwind scheme for air-mixed droplet flow in atmospheric icing. Computers and Fluids, 86:459 - 469, 2013.

[48] R. D. Keane and R. J. Adrian. Theory of cross-correlation analysis of piv images. Applied scientific research, 49(3):191-215, 1992.

[49] T. Kékesi, G. Amberg, and Wittberg L. P. Drop deformation and breakup. International Journal of Multiphase Flow, 66:1-10, 2014.

[50] J. B. Kennedy and J. Roberts. Rain ingestion in a gas turbine engine. In Proceedings of 4th ILASS Meeting, page 154, 1990.

[51] B. S. Kirk. Multidimensional assesment of modeling error in typical high-speed wind-tunnel heat-transfer data-reduction schemes. Journal of Thermophysics and Heat Transfer, 23(1):187-191, 2009.

[52] T. G. Kolda and B. W. Bader. Tensor decompositions and applications. SIAM review, 51(3):455-500, 2009.

[53] R. Krechetnikov. Rayleigh-taylor and ritchtmyer-meshkov instabilities of flat and curves interfaces. Journal of Fluid Mechanics, 625:387-410, 2009.

[54] S. A. Krzeczkowski. Measurement of liquid droplet disintegration mechanism. International Journal of Multiphase Flow, 6:227-229, 1980.

[55] A. Lampton and J. Valasek. Prediction of icing effects on the lateral/directional stability and control of light airplanes. Aerospace Science and Technology, 23(1):305 - 311, 2012. 35th ERF: Progress in Rotorcraft Research.

[56] W. R. Lane. Shatter of drops in stream of air. Industrial and Engineering Chemistry, 43(6):13121317, June 1951.

[57] B. Laschka and R. E. Jesse. Ice accretion and its effects on aerodynamics of unprotected aircraft components. Technical Report 15, AGARD Advisory Report 127, November 1978.

[58] M. W. Lee, J. J. Park, M. M. Farid, and S. S. Yoon. Comparison and correction of the drop breakup models for stochastic dilute spray flow. Applied Mathematical Modelling, 36(9):4512-4520, 2012. 
[59] G. Luxford. Experimental and Modelling Investigation of the Deformation, Drag and Break-up of Drizzle Droplets subjected to Strong Aerodynamics Forced in relation to SLD Aircraft Icing. PhD thesis, Cranfield University, 2005.

[60] G. Luxford, D. W. Hammond, and P. Ivey. Modelling, imaging and measurement of distortion, drag and break-up of aircraft-icing. volume 75, Reno, Nevada, January 2005. 43rd AIAA Aerospace Sciences Meeting and Exhibit.

[61] S. T. McClain, D. Reed, M. Vargas, R. E. Kreeger, and J.C. Tsao. Ice roughness in short duration SLD icing events. In 6th AIAA Atmospheric and Space Enviroments Conference, Atlanta, June 2014. AIAA 2014-2330.

[62] G. M. H. Meesters. Mechanisms of droplet formation. TU Delft, Delft University of Technology, 1992.

[63] G. Mingione, D. Guffond, and C. Tropea. Extice: Extreme icing environment. SAE International 2011-38-0063, 2011.

[64] K. Nakakita, S. Nadarajah, and W. Habashi. Towards real-time aero-icing simulation of complete aircraft via FENSAP-ICE.

[65] J. C. Ni, W.-C. Cheng, and L. Ge. A simple data reduction method for pumping tests with tidal, partial penetration, and storage effects. Soils and Foundations, 53(6):894-902, 2013.

[66] J. A. Nickerson and T. W. Sloan. Data reduction techniques and hypothesis testing for analysis of benchmarking data. International journal of production research, 37(8):1717-1741, 1999.

[67] P. J. O'Rourke and A. A. Amsden. The TAB method for numerical calculation of spray droplet breakup. Technical report, SAE Technical Paper, 1987.

[68] N. Otsu. A threshold selection method from gray-level histograms. IEEE TRANSACTIONS ON SYSTREMS, MAN, AND CYBERNETICS, 9(1), 1979.

[69] R. L. Panton. Incompressible Flow. John Wiley and Sons, New Jersey, USA, 2013.

[70] W. P. Partridge and M. Keyhani. A generalized procedure for reducing interferometric data. Experimental thermal and fluid science, 8(1):112-117, 1994.

[71] P. D. Patel and T. G. Theofanous. Hydrodynamic fragmentation of drops. Journal of Fluid Mechanics, 23:103-207, 1981.

[72] M. Pilch. Acceleration induced fragmentation of liquid drops. $\mathrm{PhD}$ thesis, University of Virginia, Charlottesville Va, 1981.

[73] M. Pilch and C. A. Erdman. Use of breakup time data and velocity history data to predict the maximum size of stable fragments for acceleration-induced breakup of a liquid drop. Internation Journal of Multiphase Flow, 13(6):741-757, 1987.

[74] A. K. Prasad. Particle image velocimetry. CURRENT SCIENCE-BANGALORE, 79(1):51-60, 2000.

[75] M. Raffel, C. Willert, S. Werely, and J. Kompenhans. Particel image velocimetry-a practical guide 2nd edition, 2007. 
[76] S. Rainieri, F. Bozzoli, and G. Pagliarini. Wiener filtering technique applied to thermographic data reduction intended for the estimation of plate fins performance. Experimental Thermal and Fluid Science, 28(2):179-183, 2004.

[77] A. A. Ranger and J. A. Nicholls. Aerodynamics shattering of liquid drop. volume 68-83, New York, New York, January 22-24 1968. AIAA 6tn Aerospace Sciences Meeting.

[78] A. A. Ranger and J. A. Nicholls. Atomization of liquid droplets in a convective gas stream. International Journal of Heat and Mass Transfer, 15(6):1203-1211, 1972.

[79] L. Rayleigh. On the instability of jets. Proceedings of the London mathematical society, 1(1):4-13, 1878.

[80] T. C. S. Rendall and C. B. Allen. Finite-volume droplet trajectories for icing simulation. International Journal of Multiphase Flow, 58:185 - 194, 2014.

[81] G. Rosas-Cholula, J. M. Ramirez-Cortes, V. Alarcon-Aquino, P. Gomez-Gil, J. J. Rangel-Magdaleno, and C. Reyes-Garcia. Gyroscope-driven mouse pointer with an EMOTIV $®$ EEG headset and data analysis based on empirical mode decomposition. Sensors, 13(8):10561-10583, 2013.

[82] I. Sher and E. Sher. Analytical criterion for droplet breakup. Atomization and Sprays, 21(12):1059-1063, 2011.

[83] M. Shimura and M. Yamamoto. Comparative investigation on effect of droplet deformation model on sld icing. In SAE Technical paper 2015-01-2117, 2015. doi:10.4271/2015-01-2117.

[84] A. B. Sichani and M. D. Emami. A droplet deformation and breakup model based on virtual work principle. Physics of Fluids, 27(032103):1-35, 2015. doi:10.1063/1.4943809.

[85] J. Tan, M. Papadakis, and M. K. Sampath. Computational study of large droplet breakup in the vicinity of an airfoil. Technical Report 42, DOT/FAA/AR, 2005.

[86] S. C. Tan. Effects of large droplet dynamics on airfoil impingement characteristics. AIAA paper 2005-74, 2005.

[87] S. C. Tan and M. Papadakis. General effects of large droplet dynamics on ice accretion modeling. In 41 st aerospace Sciences Meeting and Exhibit, Reno, Nevada, January 2003. AIAA.

[88] G. I. Taylor. The Shape and Acceleration of a Drop in a High Speed Air Stream, volume III of The Scientific Papers of G. I. Taylor. University Press, Cambridge, 1963.

[89] S. Temkin and H. K. Mehta. Droplet drag in an accelerating and decelerating flow. Journal of Fluid Mechanics, 116:297-313, 1982.

[90] T. G. Theofanous. Aerobreakup of newtonian and viscoelastic liquids. Annual Review of Fluid Mechanics, 43:661-690, 2011. 
[91] T. G. Theofanous and G. J. Li. On the physics of aerobreakup. Physics of fluids, 20:052103, 2008.

[92] T. G. Theofanous, G. J. Li, and T. N. Dinh. Aerobreakup in rarefied supersonic gas flow. Journal of fluids engineering, 126:516-527, 204.

[93] M. Vargas and A. Feo. Deformation and breakup of water droplets near an airfoil leading edge. Journal of Aircraft, 48:1749-1765, 2011. IF:0.538. Q3.

[94] P. V. Vukoslacevic. A hot-wire probe configuration and data reduction method to minimize velocity gradient errors for simultaneous measurements of three velocity components in turbulent flows. Experimental in Fluids, 53:481-488, 2012.

[95] C. Wang, S. Chang, and H. Wu. Theretical modeling of spray drop deformation and brealup in the multimode breakup regime. Atomization and Sprays, 2014. doi:10.1615/AtomizSpr.2015011771.

[96] C. Wang, S. Chang, H. Wu, and J. Xu. Modeling of droplet breakup in the bag breakup regime. Appl. Phys. Lett., 104:154107-1 - 154107-4, 2014.

[97] C.-C Wang, R. L. Webb, and K.-Y. Chi. Data reduction for air-side performance of fin-and-tube heat exchangers. Experimental Thermal and Fluid Science, 21:218-226, 2000.

[98] P. D. Weidman. Non-axisymmetric homann stagnation-point flows. Journal of Fluid Mechanics, 702:460-469, 72012.

[99] F. M. White and I. Corfield. Viscous fluid flow, volume 3. McGraw-Hill New York, 1991.

[100] A. Wierzba. Deformation and breakup of liquid drops in a gas stream. Experiments in fluids, 9:59-64, 1990.

[101] A. Wierzba and K. Takayama. Experimentala investigation of the aerodynamic breakuo of liquid drops. AIAA Journal, 26(11):1329-1335, November 1988.

[102] H. E. Wolfe and W. H. Andersen. Kinetic mechanism and resultant droplet sizes of the aerdynamic breakup of liquid drops. Technical Report 0395-04(18)SP, Aerojet General Corporation, Resear And Engineering Division, 11711 Woodr. ff Avenue, Downe, Califonia, April 1964. Prepared under D. S. Army Chemical Center Contract DA-18 108-405 CML-829.

[103] D. Zeppetelli and W. D. Habashi. In-flight risk management through computational fluid dynamics. Journal of aircraft, 49(2):611-621, March-April 2012. DOI: $10.2514 / 1 . C 031609$. 